\title{
Theoretical investigation of von Braun and von Braun-like reactions
}

\author{
François Couty ${ }^{a}$, Oleg N. Burov ${ }^{b}$, Mikhail E. Kletskii ${ }^{b}$, Anton V. Lisovin ${ }^{b}$, Karen Wright ${ }^{a}$, Bruno \\ Drouillat $^{a}$, Sergey V. Kurbatov ${ }^{b}$ \\ anstitut Lavoisier, UMR CNRS 8180, Université de Versailles St Quentin-en- \\ Yvelines, Versailles Cedex, France \\ ${ }^{b}$ Department of Chemistry, Southern Federal University, Rostov-on-Don, Russia
}

\section{ABSTRACT}

The von Braun reaction, discovered at the dawn of the past century, consists of the reaction between a tertiary amine and cyanogen bromide. It leads to the cleavage of a $\mathrm{C}-\mathrm{N}$ bond with the formation of an $\mathrm{N}$-dialkylcyanamide and an alkyl bromide and has been extensively used in organic synthesis. A detailed in silico study (PCM/DFT/B3LYP/6-31++G(d,p) calculations) of this venerable reaction has shown that in the first stage a zwitterionic adduct with a multi-bonded bromine atom is formed. The widely accepted mechanism involving an $\mathrm{S}_{\mathrm{N}} 2$ reaction occurs in the second step, thus accounting for its selectivity. Quantum chemical calculations were performed for the von Braun-like reactions in systems formed by cyclic tertiary amines ( $N$-alkyl azetidines). In these cases, the first stage is almost the same as in the classical von Braun processes, and selective $S_{N} 2$ mechanisms can occur in the second step.

\section{INTRODUCTION}
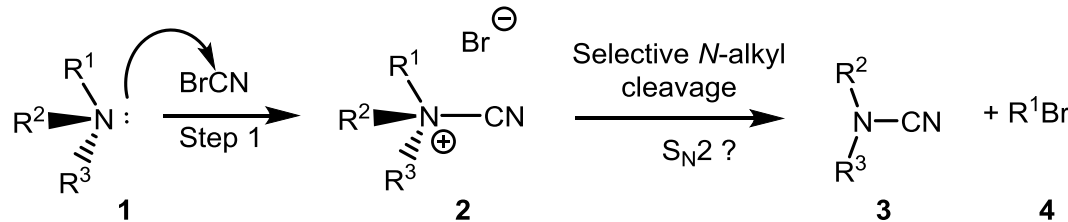

Scheme 1. Classical mechanism of the von Braun reaction

The von Braun reaction was first discovered in 1900 by Julius von Braun, at the University of Göttingen[1] in the early stages of his career (he was 25 years old). It is interesting to note that this reaction was discovered independently and published in the same journal less than a month after von Braun by Roland Scholl and Wilhelm Nörr in Karlsruhe,[2] but von Braun left his name to this reaction, being the first inventor, and he studied it thoroughly over the following three decades of his career. This process involves $\mathrm{C}-\mathrm{N}$ cleavage in a tertiary amine $\mathbf{1}$ upon reaction with cyanogen bromide, leading to a $\mathrm{N}$-dialkylcyanamide $\mathbf{3}$ and an alkyl bromide 4 (Scheme 1). The von Braun reaction has been thoroughly studied over the years, and a large number of the issues regarding selectivity in the acyclic series (preferred cleavage of $\mathrm{R}^{1-3}$ in 1 ) or in cyclic amines (preferred ring cleavage against exocyclic $N$-substitution) have been reviewed in a recent monograph by Hageman.[3] The mechanism of the reaction has been widely accepted as a two-step sequence involving firstly formation of an intermediate zwitterion $\mathbf{2}$ (which has never been unambiguously characterized), which evolves to $\mathbf{3}$ and $\mathbf{4}$. This second step, considering its stereospecificity observed in some cases,[4] as well as the scarce kinetic data, $[5,6]$ is accepted to follow a $S_{N} 2$ mechanism. From an historical viewpoint, the von Braun reaction soon appeared as a powerful tool for selective $\mathrm{N}$-alkyl cleavage in tertiary amines and was especially used as a chemical tool for the structural 
determination of alkaloids. Its synthetic use, especially as an $\mathrm{N}$-demethylation reagent has, however, declined, as chloroformates proved safer and often more selective. $[7,8]$
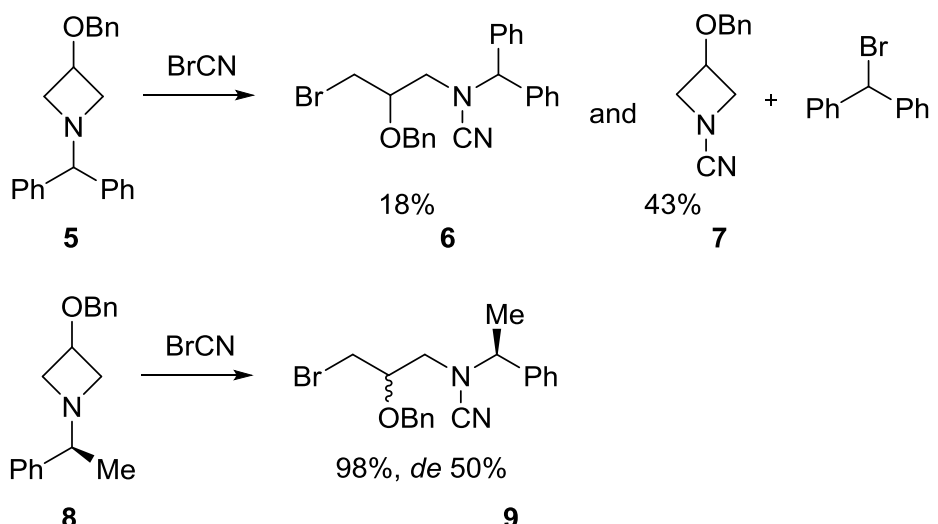

Scheme 2. The von Braun reaction of azetidines

We recently became interested in this old reaction, having demonstrated that it leads to the ring cleavage of strained azetidines in a regioselective manner to give, for example, 3-bromo cyanamide 6 from azetidine 5, or in a diastereoselective manner, to give $\mathbf{9}$ with a significant level of diastereoinduction[9] (Scheme 2, equations [1] and [2]).

Moreover, the produced 3-bromocyanamides were demonstrated to be new and highly versatile biselectrophilic building blocks, notably for the preparation of guanidines.[10] In order to determine the key parameters allowing an understanding of these reactions, and especially with the aim of optimizing the level of diastereoselectivity in ring opening leading to $\mathbf{9}$, we needed to have a clear idea of its mechanism. Besides, we soon realized that despite its long history, no theoretical calculations on the von Braun reaction have been published, to the best of our knowledge.

This article thus reports a detailed in silico study of the reaction, first with 1-ethyl-1methylpropylamine $1\left(R^{1}=\mathrm{Me}, \mathrm{R}^{2}=\mathrm{Et}, \mathrm{R}^{3}=\mathrm{Pr}\right.$, vide infra structure 10$)$, as reported initially by von Braun, who observed the selective cleavage of the $\mathrm{N}$-methyl group, and then with simplified analogues of $\mathbf{5}$ and $\mathbf{8}$, for which significant $\mathrm{N}$-benzhydryl cleavage on one hand, and exclusive and diastereoselective ring opening on the other hand were observed. The key objective of this study was to understand the effect of the nature of the substituent at the nitrogen atom on the von Braun reaction mechanism, which in some cases results in only acyclic products and in others to a mixture of two products (substitution at the nitrogen atom and azetidine ring opening).

\section{COMPUTATIONAL METHODS}

Density functional theory (DFT) calculations were performed using B3LYP exchange-correlation functionals,[11-13] together with the standard6-31G(d,p) basis set.[14] Geometry optimization was carried out using Berny's analytical gradient optimization method. Stationary points of the reaction minimum energy paths (MEPs) were characterized by frequencies calculations. MEPs were obtained via the gradient descent method from the transition states in forward and reverse directions of the transition vectors. 
The efficiency of DFT treatment for the reactions involving strained cyclic systems was demonstrated by us earlier.[15] When choosing the $6-31++G(d, p)$ basis for the theoretical study of such systems by the B3LYP functional, we were guided by literature data for the calculation of bromine containing organic molecules: G-bases of types 6-31 and 6-311 give a small general error and good results (geometric characteristics), even without taking into account the relativistic effects.[16,17]

We also considered the influence of noncovalent interactions on the mechanisms of the studied reactions. To do this, in DFT calculations corrections for dispersion interactions according to the scheme[18] were included. It turned out that taking into account these corrections (PCM/DFT/B3LYP/6-31++G(d,p)/GD3BJ calculations at different temperatures) does not affect qualitatively on the conclusions of present work. Some important results are given in the Supporting Information (eg, Scheme S1, Figure S1).

The solvent effects have been considered in the context of a polarizable continuum model (PCM),[19-22] and $\mathrm{CHCl}_{3}$ was chosen as solvent, which has been frequently used in such reactions. All computations were carried out using the Gaussian 09 suite of programs.[23] Also, we performed a topological analysis of electron density distribution function according to the "atoms in molecules" (AIM) model.[24]

\section{RESULTS AND DISCUSSION}

\section{1. von Braun reaction mechanisms}

The classical explanation of the von Braun reaction mechanism lies in its two-step character. According to this description, along the reaction pathway there are two nucleophilic substitutions. First, the alkylamine 10 reacts with cyanogen bromide $\mathbf{1 1}$, yielding a quaternary ammonium salt $\mathbf{1 2}$, which rearranges in the next step by splitting to form a bromo-substituted alkane and a dialkylcyanamide, respectively (Scheme 3 ).

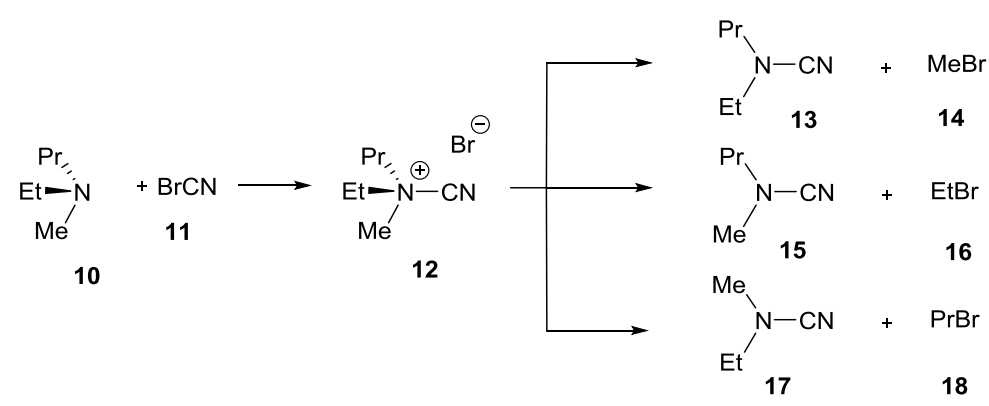

Scheme 3. Possible paths of the von Braun reaction

It is well known that the methyl group is preferentially lost from this system, while heavier alkyl substituents are worse leaving groups.[1] In our calculations, the possible pathways of the processes were studied for the interaction of 1-ethyl-1-methylpropylamine $\mathbf{1 0}$ with cyanogen bromide $\mathbf{1 1}$. Calculations have shown that for the initial stage, only two preferred relative orientations of the reagents in the initial supramolecular complex $\mathbf{1 2}$ exist-12a and $\mathbf{1 2 b}$ (Scheme 4).

The formation of $\mathbf{1 2 a}$ and $\mathbf{1 2 b}$ requires a lot of activation energy ( $\geq 20 \mathrm{kcal} / \mathrm{mol}$, see transition states TSA, TSB in Figure 1); however, these barriers may be overcome at the high temperatures used in the early experiments.[1] The structures of transition states TSA and TSB differ in the orientation of the 
cyanogen bromide molecule relative to tertiary amine. In the case of TSA, the bromine atom is closer to the propyl group, in the case of TSB closer to the methyl group. Energetically more favorable transition state TSB is more compact due to shorter $\mathrm{C}-\mathrm{N}$ distance (Figure 1).

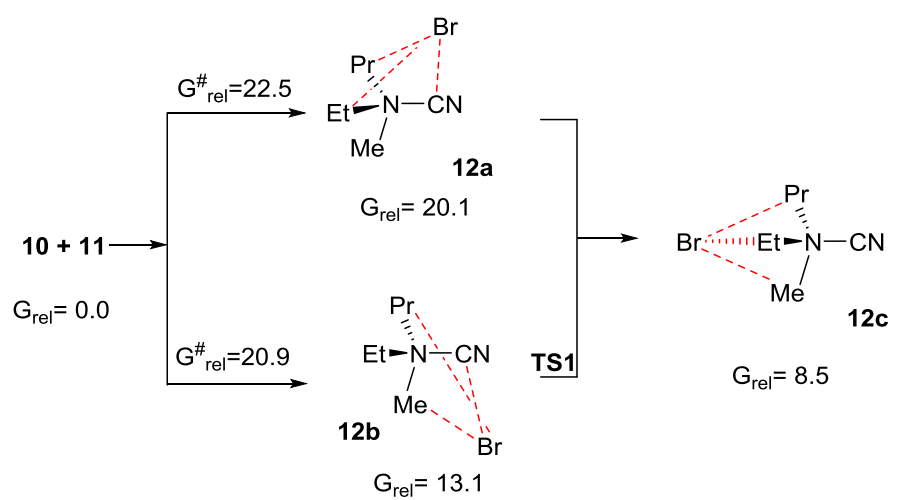

Scheme 4. Formation of the intermediate $12 \mathrm{c}$. $\mathrm{G}_{\mathrm{rel}}$ - relative Gibbs free energies in $\mathrm{kcal} / \mathrm{mol}$. The sum of total free energies for the isolated reactants was assumed as a reference.

Despite the fact that Hessians for the complexes 12a and 12b do not have negative eigenvalues $(\lambda=$ $0)$, these systems are kinetically rather unstable: $2.7 \mathrm{kcal} / \mathrm{mol}$ is required for the rearrangement into the more stable intermediate form $\mathbf{1 2 c}$ for isomer $\mathbf{1 2 b}$ via TS1, and there is practically no barrier for system 12a.
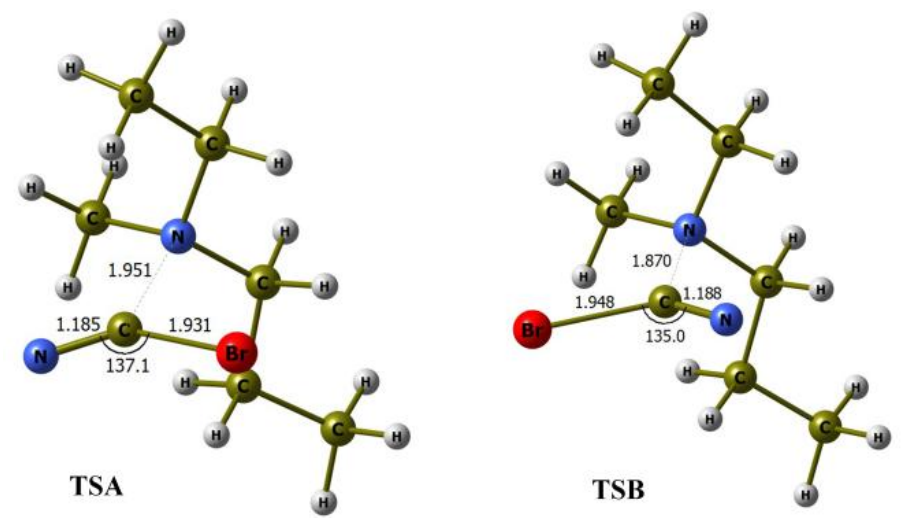

Figure 1. Transition states TSA, TSB geometries. The main interatomic distances are in $\AA$, valence angles in degrees. PCM/DFT/B3LYP/6-31++G(d,p) calculations in $\mathrm{CHCl}_{3}, 100^{\circ} \mathrm{C}$.

According to AIM calculations, $\mathbf{1 2 a}, \mathbf{1 2} \mathbf{b}$, as well as $\mathbf{1 2 c}$ contain a tris-coordinated bromine atom (Figure 2). The charge values on bromine atoms in complexes 12a and $\mathbf{1 2 b}$ account for $-0.850 \bar{e}$ and $-0.864 \bar{e}$, respectively. It means that intermediate systems $\mathbf{2}$ or $\mathbf{1 2}$ may be considered as zwitterionic. According to the calculations, systems $\mathbf{1 2 a}, \mathbf{1 2 b}$, and $\mathbf{1 2 c}$ are stabilized relative to the pair of separated ions by not less than $20 \mathrm{kcal} / \mathrm{mol}$. 

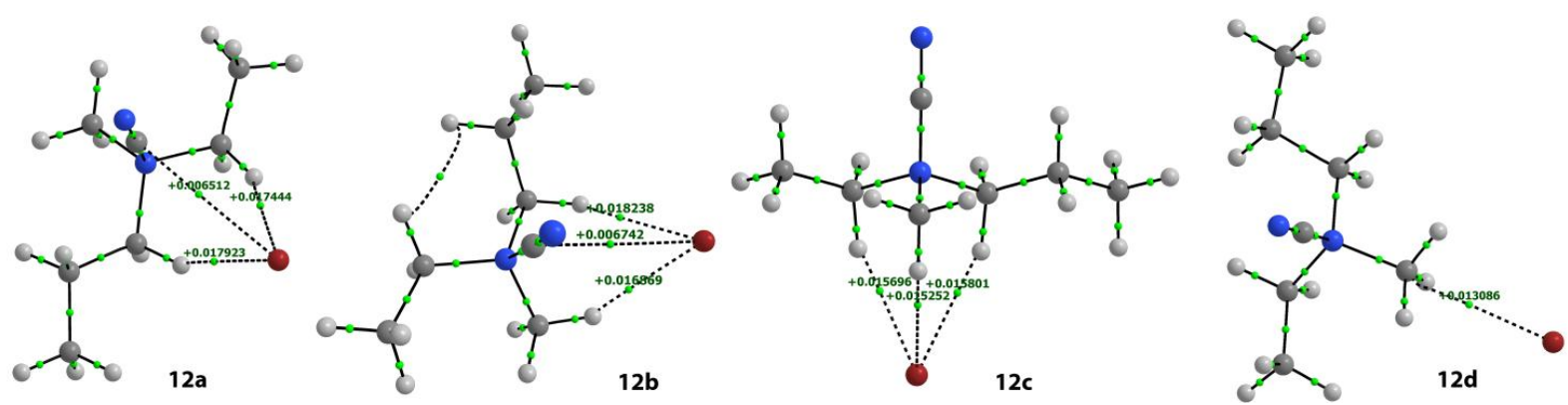

Figure 2.The attractive forces in systems $\mathbf{1 2 a - 1 2 d}$ according to AIM calculations. $\rho(\mathrm{rc})$ - Electron density values.

The second step of the von Braun reaction (decomposition of intermediate 12c via the elimination of alkyl bromides) may proceed in various ways. The reaction's development depends on the direction of intramolecular nucleophilic attack at the $\alpha$-carbon atoms of three alkyl radicals (Scheme 5). According to computational data, the removal of ethyl and propyl groups occurs directly from the intermediate 12c, while methyl removal occurs only from intermediate $12 \mathrm{~d}$, which can easily be formed in the rearrangement $\mathbf{1 2 c} \rightarrow \mathbf{1 2 d}$ (Scheme 6, TS2). At the same time, the final products $\mathbf{1 3}+$ $\mathbf{1 4}$ are slightly less thermodynamically stable compared to products $\mathbf{1 5}+\mathbf{1 6}$ or $\mathbf{1 7}+\mathbf{1 8}$ (Scheme 3, right side of Figure 3 ).<smiles>CCP(CC)N(C#N)C=CBr</smiles><smiles>CC(=P)N(C)C(C)(C)N</smiles><smiles>CCC(Br)C(Br)N(C)N</smiles>

Scheme 5. Expected bipyramidal structures for inversion processes.

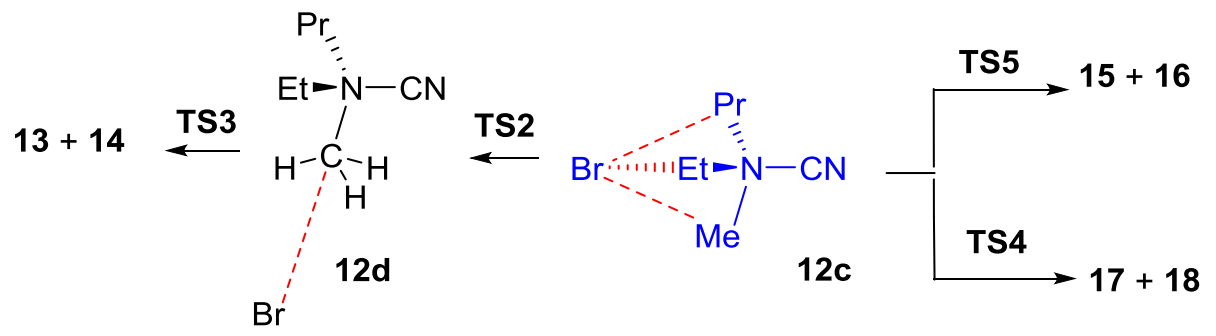

Scheme 6. von Braun reaction paths with inversion of configuration.

According to the calculations, in all three cases of $\mathrm{C}-\mathrm{N}$ bond breaking, inversion of the carbon atom configuration occurs, as shown by the specific orientation of the bromine atom and the $\mathrm{sp}^{3}$-hybrid center accompanied by formation of trigonal bipyramidal structures, where nucleophile and nucleofuge are located at approximately diaxial positions (Scheme 5). 
In system 12c, the relative orientation of the bromine atom and $\mathrm{CN}$ group is indeed biaxial: the $\mathrm{N}-\mathrm{C}-\mathrm{N}$ angle is about $180^{\circ}$ ? (Figures 2, 4 and Supporting Information). Also, the $\mathrm{Br}-\mathrm{C}$ distance for TS5 is equal to $2.620 \AA$, which is almost $0.15 \AA$ less than in the cases of TS3 and TS4 (Figure 4).

Another path for the reorganization of system 12c is a high-barrier intramolecular front-side substitution with retention of configuration at the $\alpha$-carbon atom[25-27] (Figures 3, 5). Such processes are typical for heavy nucleophiles with easily polarized nucleophilic centers (soft nucleophiles like $\mathrm{HS}^{-}, \mathrm{H}_{2} \mathrm{P}^{-}$, halides ${ }^{-}$) and hard nucleofuges (like $\mathrm{H}_{3} \mathrm{O}^{+}, \mathrm{F}, \mathrm{NR}_{1} \mathrm{R}_{2} \mathrm{R}_{3} \mathrm{R}_{4}{ }^{+}$).[28,29] In contrast to the previously discussed attacks with configuration inversion, in these cases additional "settings" like 12c, 12d are not required, and products 13-18 can be obtained directly from $12 \mathrm{c}$ via transition states TS6-TS8 (Figures 3, 5). It is worth noting that $\mathrm{C}-\mathrm{N}$ bond breakage here requires a much higher energy input than in the case of inversion processes (Figure 2). The $\mathrm{N}-\mathrm{CH}_{3}$ bond rupture is much more difficult; it proceeds via the TS8 transition state that is $10 \mathrm{kcal} / \mathrm{mol}$ higher in energy than TS6 when the N-Pr bond is broken, and also TS7 upon bond breakage with the ethyl substituent (Figure 3).

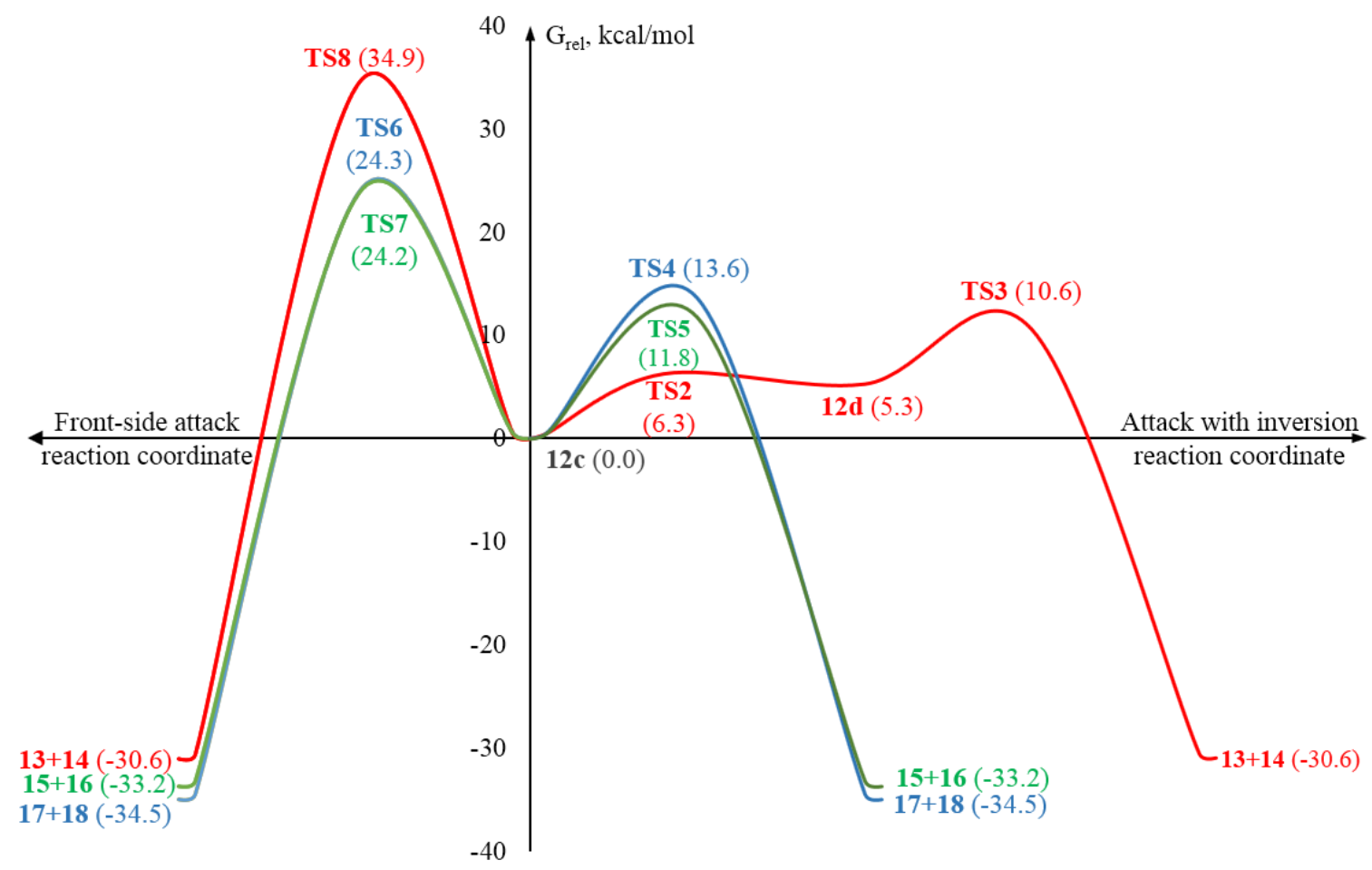

Figure 3. MEPs for the von Braun reactions. PCM/DFT/B3LYP/6-31++G(d,p) calculations in $\mathrm{CHCl}_{3}, 100$ ${ }^{\circ} \mathrm{C}$. $\mathrm{G}_{\text {rel }}$ - relative Gibbs free energies. The total free energy of $\mathbf{1 2 c}$ is assumed as reference. The temperature was chosen according to experimental data. ${ }^{[9]}$

Thus, the mechanism of the von Braun reaction presents itself as the addition of the nitrile moiety to the nitrogen atom to form a quaternary ammonium, stabilized by a multibonded bromine atom, followed by alkyl abstraction via $\mathrm{S}_{\mathrm{N}} 2$ inversion paths. Here, the elimination of the methyl group (the worst electron donor, compared to ethyl and propyl, and thus the most accessible) is kinetically more favorable. The breakage of associates like $\mathbf{1 2 c}$ or $\mathbf{1 2 d}$ via the front-side mechanism requires large energy inputs (>20 kcal/mol), and is not likely to compete with the $S_{N} 2$ inversion paths. 

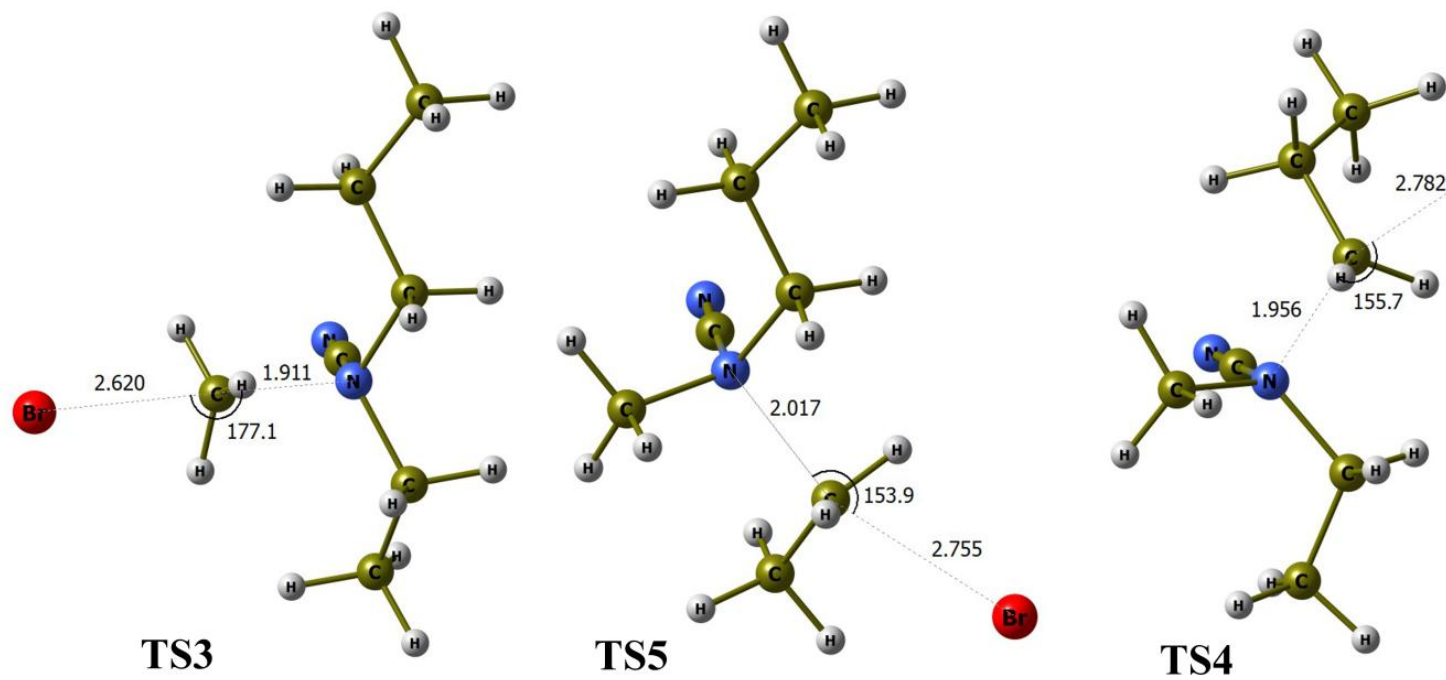

Figure 4. Transition states TS3-TS5 geometries for the processes with configuration inversion. The main interatomic distances are in $\AA$, angles in degrees. PCM/DFT/B3LYP/6-31++G(d,p) calculations in $\mathrm{CHCl}_{3}, 100^{\circ} \mathrm{C}$.

\section{2. von Braun-like reaction I}

We will now consider the von Braun-like reaction of an azetidine 19 with cyanogen bromide 11 . The principal difference between this case and the previously considered von Braun reaction is that for azetidines, opening of the strained four-member ring may occur during the substitution process. In this case (Scheme 7), the reaction may result in a mixture of products: two diastereomeric open chain products $\mathbf{2 1 a , b}$ and/or a cyanoazetidine $\mathbf{2 2}+\mathbf{1}$-bromoethylbenzene $\mathbf{2 3}$.

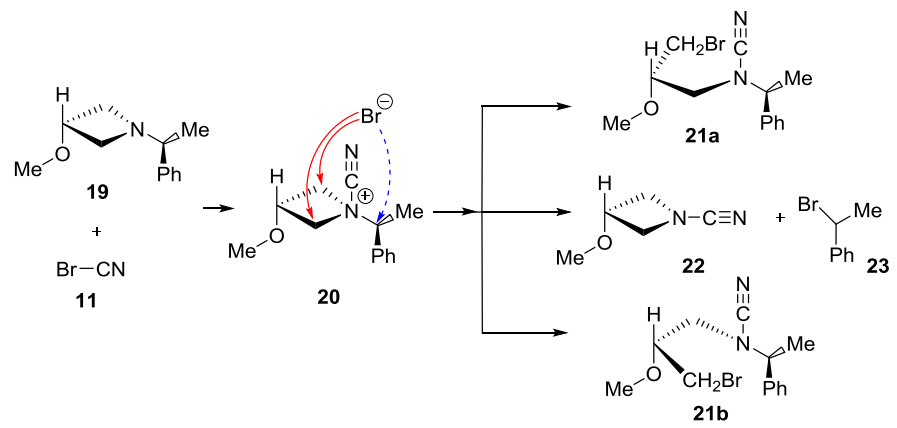

Scheme 7. General scheme for the von Braun-like reaction I. ${ }^{[9]}$

According to our calculations, at $-25^{\circ} \mathrm{C}$ the interaction of the azetidine 19 and $\mathrm{BrCN} 11$ results in the first step in the formation of tight associates 20a or 20b, as in the classical von Braun reaction (Scheme 8). Also, as earlier, the bromine atom in these associates can shift around the molecular skeleton to form several local minima that lie on a plateau on the potential energy surface (PES).

In the case of azetidine 19, when nucleophilic attacks with configuration inversion take place, the bromine atom may be located along the $\mathrm{N}-\mathrm{CH}_{2}$ cyclic bond directions (structures 20a and 20b in Scheme 8) to form two local minima (compare with system 12d), with a specific bromine coordination at the methyl group. Nevertheless, according to AIM calculations, both intermediates 20a, 20b differ from the previously described systems in the von Braun processes in that the bromine 
atom is only bis-coordinated. As can be seen in Figure 5, the bromine in both cases is bonded with the methylene unit of the azetidine and also with one hydrogen atom of the methoxy group.

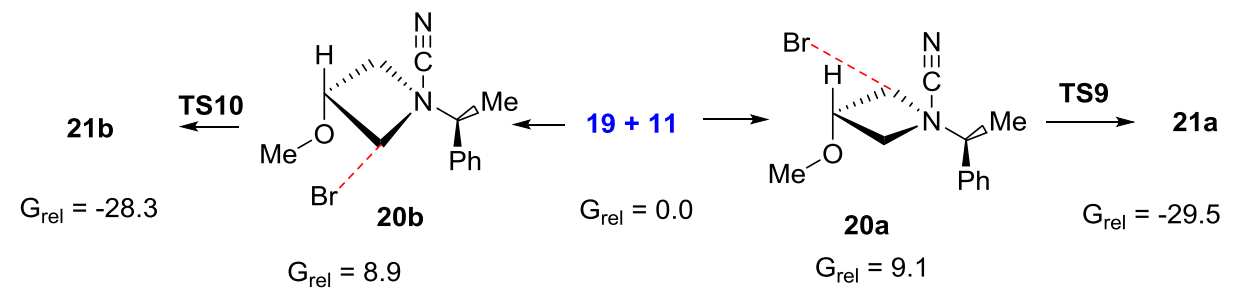

Scheme 8. $S_{\mathrm{N}} 2$ reaction paths for the von Braun-like reaction I. The sum of total Gibbs free energies in $\mathrm{kcal} / \mathrm{mol}$ of systems $\mathbf{1 9 + 1 1}$ is assumed as reference.

At the same time, in systems $20 \mathrm{a}$ and $\mathbf{2 0 b}$, loosening of the bond strength between the quaternized nitrogen and the carbon atoms of the azetidine ring is observed. In the second step of the reaction the bromine atom in systems $\mathbf{2 0 a}$ and $\mathbf{2 0 b}$ may attack practically without any energy barrier (see TS9, $\mathrm{G \#} \mathrm{rel}=0.3 \mathrm{kcal} / \mathrm{mol}, \mathrm{TS} 10, \mathrm{G} \# \mathrm{rel}=0.3 \mathrm{kcal} / \mathrm{mol}$ in Supporting Information) the carbon atom of the methylene unit, followed by ring opening. Thus, the final diastereomeric compounds $\mathbf{2 1 a}$ and $\mathbf{2 1} \mathbf{b}$ are formed.
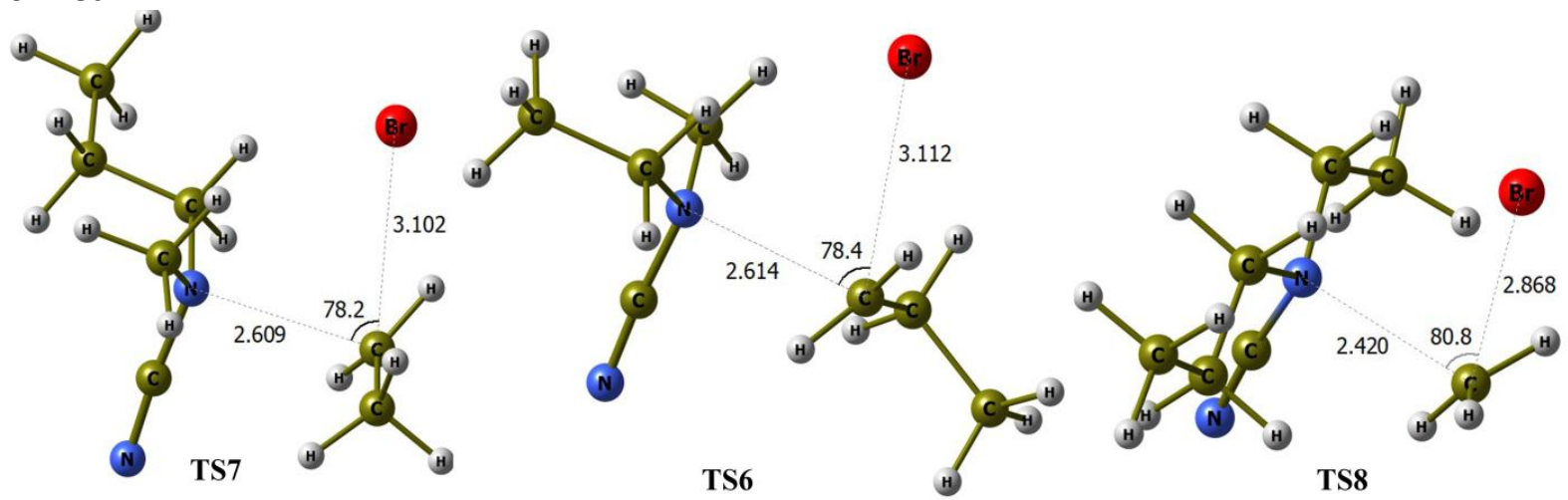

Figure 5. Transition states geometries for the front-side nucleophilic attack. The main interatomic distances are in $\AA$, angles in degrees. PCM/DFT/B3LYP/6-31++G(d,p) calculations in $\mathrm{CHCl}_{3}, 100^{\circ} \mathrm{C}$.

The lack of the third reaction complex, where the bromine atom is found near the $\alpha$-carbon of the phenethyl group (blue dotted arrow in Scheme 7), stands out. For this orientation, we found neither minima nor transition states for the process of phenethyl elimination. Therefore, according to the calculations, the direct path $(19+\mathbf{1 1}) \rightarrow(\mathbf{2 2}+\mathbf{2 3})$ with inversion of configuration does not exist. The interaction of substituted azetidine 19 and $\operatorname{BrCN} 11$ may also result in the formation of the relatively stable associate 20c, in which the bromine atom is multibonded once again and is found on the opposite side of the $\mathrm{CN}$ group (compare with trigonal bipyramid 12c). According to AIM calculations for system 20c, the bromine atom is tetracoordinated and forms bond paths with the hydrogen atoms of the methylene units, the phenethyl unit, and the ortho proton of the phenyl substituent (Figure 6). In the associate 20c, again, there is a weakening of the bond between the quaternized nitrogen atom and the phenethyl $\alpha$-carbon. 

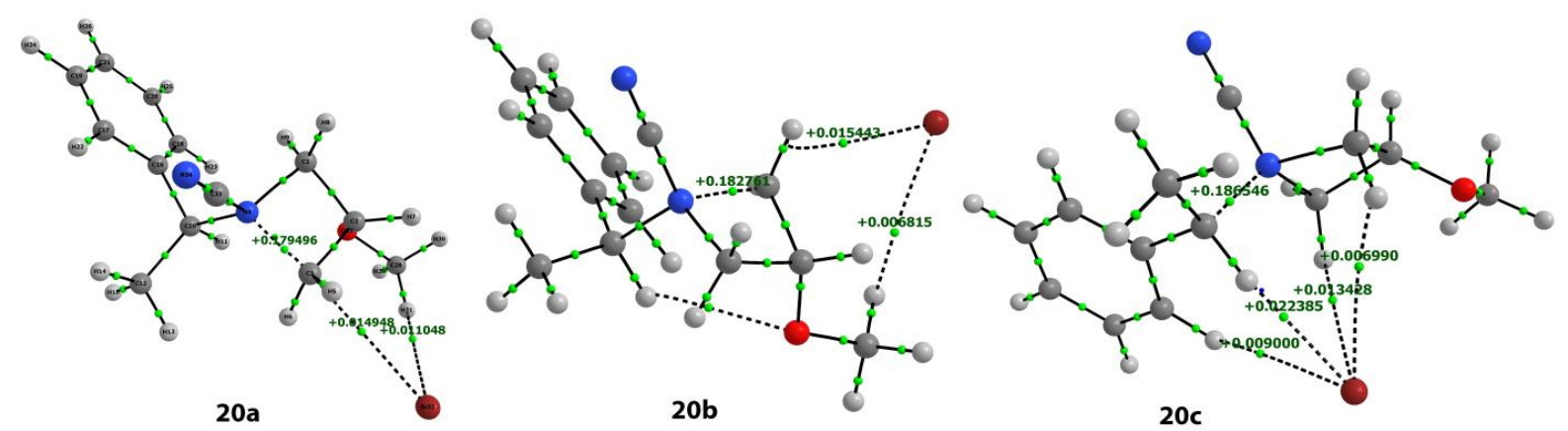

Figure 6. The attractive forces in systems 20a, 20b, and 20c according to AIM calculations. $\rho(\mathrm{rc})$ Electron density values.

Also, system 20c can be rearranged in an intramolecular nucleophilic process with retention of configuration (front-side nucleophilic attack), as shown in Scheme 9. As the azetidine ring is strained, and the phenethyl moiety is a good leaving group, the $\mathrm{N}^{+}-\mathrm{C}$ bond rupture is less energy consuming than that of the classical von Braun reaction (Figure 7). The reaction in this case develops in the same way as for the associate 12c through the transition states TS6-TS8. Thus, complex 20c is a precursor of three different products: open-chain diastereomers $\mathbf{2 1 a}, \mathbf{2 1}$ b, and products $\mathbf{2 2}+\mathbf{2 3}$.

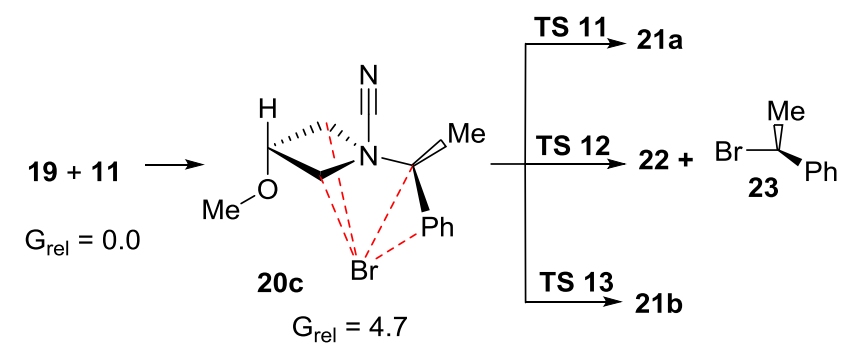

Scheme 9. Front-side nucleophilic attacks for the von Braun-like reaction I. The sum of total free energies in $\mathrm{kcal} / \mathrm{mol}$ of systems $\mathbf{1 9 + 1 1}$ is assumed as reference.

As can be seen in Figure 7, the formation of products $\mathbf{2 2}+\mathbf{2 3}$ is kinetically (TS12) the least demanding and may compete with nucleophilic attack at the cyclic carbon atom with configuration inversion (Scheme 8). Final open-chain products $\mathbf{2 1 a}$ and $\mathbf{2 1} \mathbf{b}$, formed from systems $\mathbf{2 0 a}$ and $\mathbf{2 0 b}$, are identical to the products derived from system 20c, as the methylene units are not chiral. Thus, one may observe a mixture of open-chain products $\mathbf{2 1 a}$, 21b (main products) with possible traces of mixture $22+23$

However, moving to the experimentally studied system $\mathbf{8}$, we managed to localize two reaction complexes with different arrangements of the bromine atom, as well as for the processes in Scheme 8 (Scheme 10). 


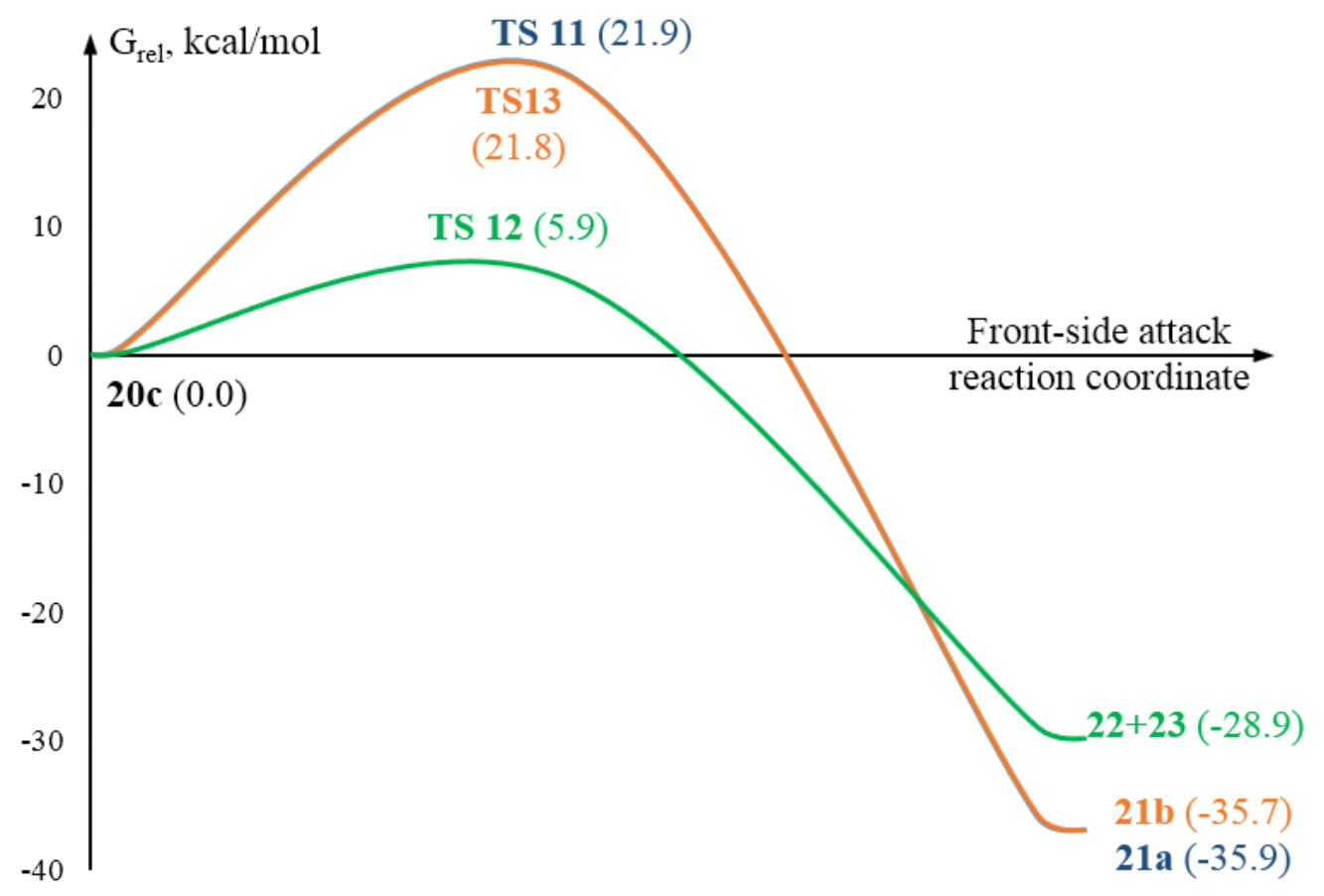

Figure 7. MEPs of the second step for the von Braun-like reaction I (configuration retention paths). PCM/DFT/B3LYP/6-31++G(d,p) calculations in $\mathrm{CHCl}_{3},-25^{\circ} \mathrm{C}^{\left[{ }^{[9]}\right.} \mathrm{G}_{\text {rel }}$ - relative Gibbs free energies. The total free energy of $\mathbf{2 0 c}$ is assumed as reference.

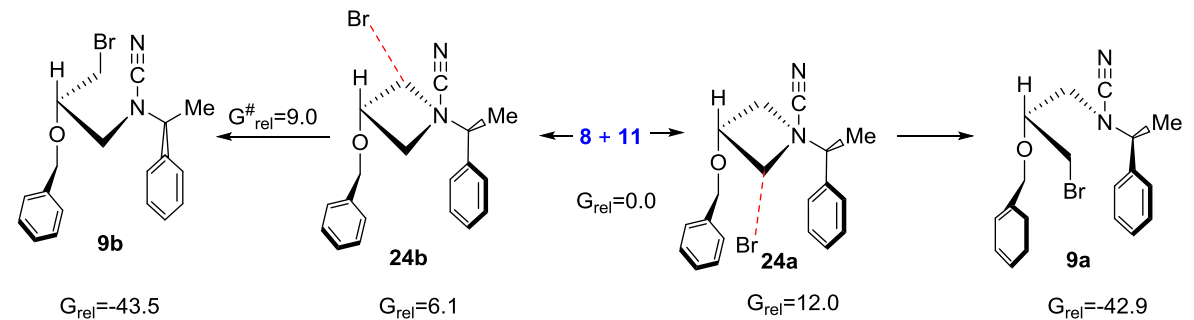

Scheme 10. Front-side nucleophilic attacks for the reaction between systems $\mathbf{8}$ and $\mathbf{1 1}$. The sum of total Gibbs free energies in $\mathrm{kcal} / \mathrm{mol}$ of systems $\mathbf{8 + 1 1}$ is assumed as reference.

The calculations suggest that complex 24a on $5.9 \mathrm{kcal} / \mathrm{mol}$ is less stable than complex $\mathbf{2 4 b}$. The opening of the four-member rings in both systems also leads to the formation of diastereomeric products $9 \mathbf{a , b}$ but in different proportions.[9] For the system 24a ring opening proceeds without an energy barrier, while for complex $\mathbf{2 4 b}$ the activation barrier is $2.9 \mathrm{kcal} / \mathrm{mol}$. According to calculations, in the case of system $\mathbf{8}$ the initial supramolecular complex like $\mathbf{2 0 c}$ with a multibonded bromine atom does not exist on the PES.

\section{3. von Braun-like reaction II}

As before, when azetidine 22 reacts with $\mathrm{BrCN}$ 11, the reaction can proceed in two major directions: azetidine ring opening with the formation of unique product $\mathbf{2 6}$, or the formation of two products bromodiphenylmethane $\mathbf{2 7}$ and cyanoazetidine $\mathbf{2 2}$ (Scheme 11). 


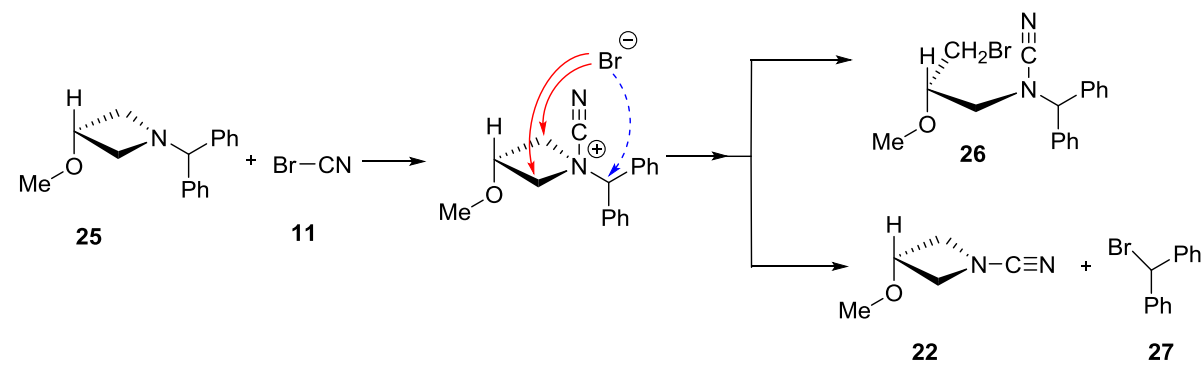

Scheme 11. General scheme of the von Braun-like reaction II

The interaction of azetidine $\mathbf{2 5}$ and cyanogen bromide $\mathbf{1 1}$ proceeds in the first step to form an associate with a relatively sterically nonrigid bromine atom. System 28a (Figure 7, Scheme 12) is stabilized by the bromine atom in a tris-coordinated form (AIM results).

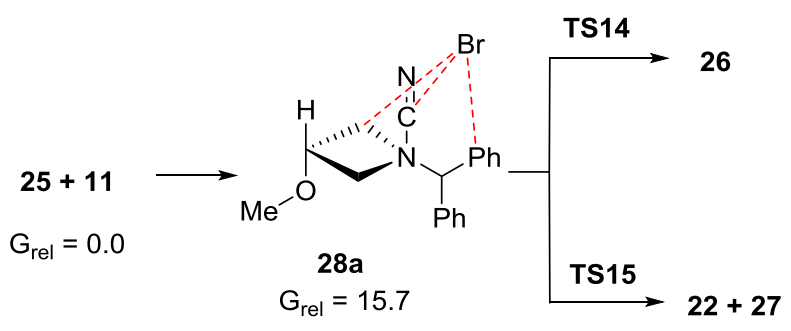

Scheme 12. Front-side nucleophilic attack in the von Braun-like reaction II. The sum of total Gibbs free energies (in $\mathbf{k c a l} / \mathbf{m o l}$ ) of systems $\mathbf{2 5 + 1 1}$ is assumed as reference.

A greater loosening of the bond paths is observed, as earlier, between the nitrogen $\mathrm{N}^{+}$atom and the diphenylmethane substituent. Intermediate $\mathbf{2 8}$ a contains a strained azetidine ring and a good leaving group, the diphenylmethyl moiety, which makes it more susceptible to breakage of the $\mathrm{C}-\mathrm{N}$ bond via the front-side path with $\mathrm{sp}^{3}$ carbon atom configuration retention.
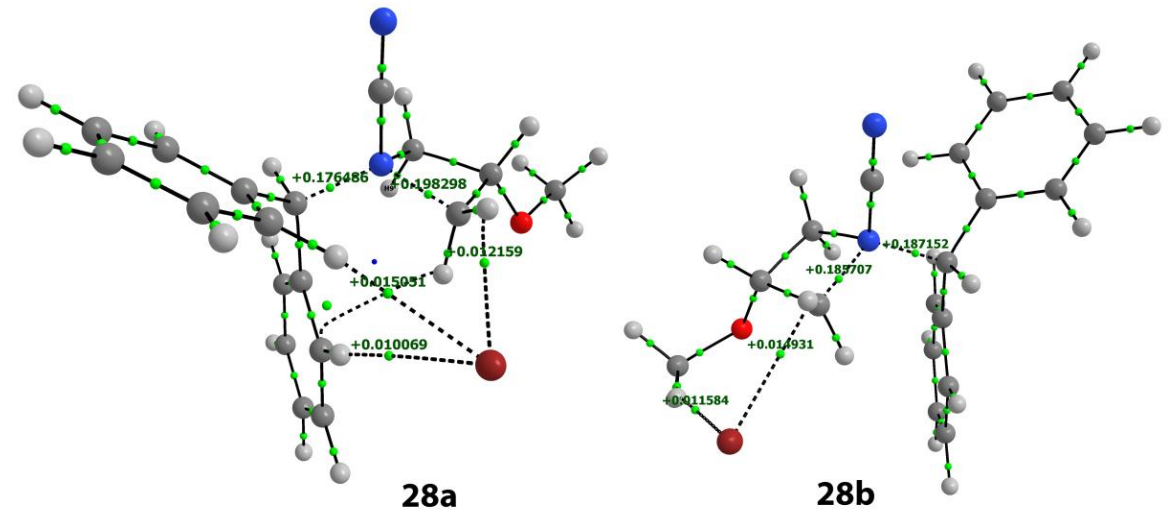

Figure $\mathbf{8}$. The attractive forces in systems $\mathbf{2 8 a}$ and $\mathbf{2 8 b}$ according to AIM calculations. $\rho(\mathrm{rc})$ - Electron density values. 
The $\mathrm{N}-\mathrm{CH}_{2}$ bond cleavage in the azetidine ring followed by bromine addition (product 26), as in the previous cases of $\mathrm{S}_{\mathrm{N}} 2$ attack with retention, requires a higher energy input than the cleavage of the diphenyl moiety (products 22, 27; Figure 9). Abstraction occurs via a low-barrier transition state, TS15. Also, as in case of the 1-(diphenylmethyl)azetidine reaction, final products $\mathbf{2 2}+\mathbf{2 7}$ formation is not accompanied by changing the configuration (front-side attack) of the $\mathrm{sp}^{3}$ hybridized carbon atom of the diphenylmethyl unit. In other words, inversion does not occur.

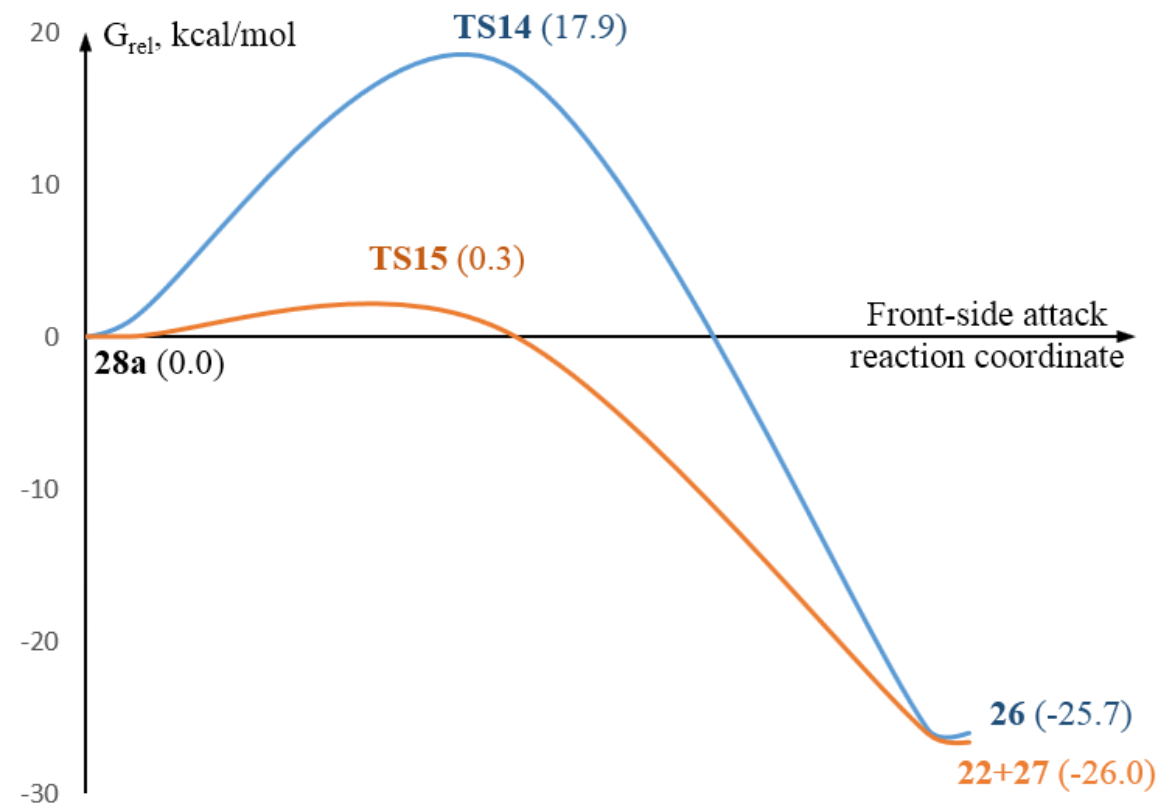

Figure 9. MEPs for the von Braun-like reaction II. Front-side attack via intermediate 28a. $\mathrm{PCM} / \mathrm{DFT} / \mathrm{B} 3 \mathrm{LYP} / 6-31++\mathrm{G}(\mathrm{d}, \mathrm{p})$ calculations in $\mathrm{CHCl}_{3}, 25^{\circ} \mathrm{C}$ [9]. $\mathrm{G}_{\text {rel }}$ - relative Gibbs free energies. The total free energy of $\mathbf{2 8 a}$ is assumed as reference.

As for the von Braun-like reaction I, the interaction of 1-(diphenylmethyl)azetidine 25 and BrCN 11 may proceed via the formation of associate $\mathbf{2 8 b}$ (Figure 8), practically of the same energy as associate 28a, in which the bromine atom is located along the $\mathrm{N}-\mathrm{CH}_{2}$ bond (Scheme 13). According to AIM calculation data, just as in associate $\mathbf{2 0 b}$, the bromine atom in system $\mathbf{2 8 b}$ is only biscoordinated and has bond paths with the carbon atom of the methylene unit and a proton of the methoxy group (Figure 9). In addition, in the associate $\mathbf{2 8 b}$ there is a weakening of the bonds of the quaternized nitrogen atom, both with the carbon atom of the diphenylmethyl substituent and the carbon atom that is attacked. In this case, the latter bond is weakened more.

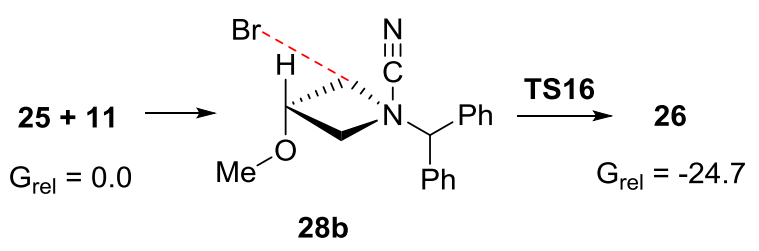

$\mathrm{G}_{\text {rel }}=16.8$

Scheme 13. Configuration inversion paths for the 1-(diphenylmethyl)azetidine reaction. The sum of total Gibbs free energies (in $\mathrm{kcal} / \mathrm{mol}$ ) of mixture $\mathbf{2 5 + 1 1}$ is assumed as reference.

Associate $\mathbf{2 8 b}$ rearranges into open-chain product $\mathbf{2 6}$ practically without an activation barrier (TS16 is only $0.3 \mathrm{kcal} / \mathrm{mol}$ higher than $\mathbf{2 8 b}$ ) and according to the intramolecular mechanism with 
configuration inversion. Interestingly, for the systems $\mathbf{2 0}$ and $\mathbf{2 8}$ on the respective MEPs there is no stationary point like $\mathbf{1 2 d}$ from which it could evolve by the way of benzhydryl fragment separation with inversion. Thus, in the von Braun-like reaction II there is a competition between ring opening with inversion and the path with configuration retention, leading to products $\mathbf{2 2}$ and $\mathbf{2 7}$.

Using the Boltzmann distribution it can be shown that for the model products $\mathbf{2 6}$ and $\mathbf{2 2}+\mathbf{2 7}$ (for the OMe-substituted system) computations give a ratio of 1:3.7. At the same time, experimental observation gives a ratio of 1:2.4 for a similar OBn-substituted system (products 6 and 7, Scheme 2). In both cases, the preferred pathway leads to azetidine formation. In accordance with experimental findings,[9] the present calculations show that such substrates preferentially form products resulting from a front-side attack.

\section{CONCLUSIONS}

At the first stage of the von Braun and von Braun-like reactions I and II, molecular associates with covalent $\mathrm{N}-\mathrm{CN}$ bonding and a multicoordinated bromine atom are formed. These associates are sterically nonrigid systems, allowing low-energy shifts of the bromine atom relative to the molecular skeletons. In every case, charge separations in the molecular associates are insignificant. The mechanism of the von Braun and von Braun-like reactions for azetidine substrates critically depends on the location of the bromine atom in the intermediate molecular associate.

All reactions of tertiary cyanamide formation are one-stage. The mechanisms of all reactions depend not only on the nature of the outgoing group, but also on the ability of the ring to be opened. In von Braun reactions of azetidines, the higher the nucleofugicity of the substituent at the nitrogen atom, the more likely it is that the substituted cyclic product will be formed.

It is obvious that electron acceptor substituents at a quaternized nitrogen atom reduce the contribution of $\mathrm{AOs}$ responsible for the strength of the $\mathrm{N}-\mathrm{C}$ bond, and therefore facilitate the separation of the substituent in $\mathrm{S}_{\mathrm{N}} 2$-type reactions with four-member ring preservation. Electrondonating substituents, on the contrary, increase the overlapping of $\mathrm{N}$ and $\mathrm{C}$ atoms AOs, and von Braun and von Braun-like reactions occur with difficulty, as is the case in the classical process or in von Braun-like reactions I, where the donor effect of the methyl group and acceptor effect of the phenyl group cancel each other out.

Thus, the main conclusion from the calculations is that the von Braun reaction is an effective method for selective opening of the azétidines ring, but only in those cases where the nitrogen atom in the four-member ring is bonded to a substituent of low electronegativity. Otherwise, during the opening of the ring, detachment of such a substituent by the bromine atom can compete with ring opening, leading to the formation of a mixture of products.

\section{ACKNOWLEDGMENTS}

University of Versailles St-Quentin en Yvelines and the CNRS are acknowledged for generous support. 


\section{REFERENCES}

[1] J. V. Braun, Chem. Ber. 1900, 33, 1438.

[2] R. Scholl, W. Nörr, Chem. Ber. 1900, 33, 1550.

[3] H. A. Hageman, Org. React. 2011, 7(4), 198.

[4] W. S. McCall, T. A. Grillo, D. L. Comins, Org. Lett. 2008, 10, 3255.

[5] G. Fodor, S. Abidi, Tetrahedron Lett. 1971, 12(18), 1368.

[6] R. O. Griffith, R. S. Jobin, A. McKeown, Trans. Faraday Soc. 1938, 34, 316.

[7] J. H. Cooley, E. J. Evain, Synthesis 1989, 1989(1), 1.

[8] R. A. Olofson, J. T. Martz, J. P. Senet, M. Piteau, T. J. Malfroot, J. Org. Chem. 1984, 49, 2081.

[9] K. Wright, B. Drouillat, L. Menguy, J. Marrot, F. Couty, Eur. J. Org. Chem. 2017, 47, 7195.

[10] K. Wright, B. Drouillat, L. Menguy, J. Marrot, F. Couty, Eur. J. Org. Chem. 2019, 49, 112.

[11] A. D. Becke, Phys. Rev. A 1988, 15, 3098.

[12] A. D. Becke, J. Chem. Phys. 1993, 98, 5648.

[13] C. Lee, W. Yang, R. G. Parr, Phys. Rev. B 1988, 37, 785.

[14] W. J. Hehre, L. Radom, P.v. R. Schleyer, J. A. Pople, Ab Initio Molecular Orbital Theory, Wiley, New York, NY 1986.

[15] F. Couty, M. Kletskii, J. Mol. Struct. Theochem. 2009, 908(1-3), 26.

[16] J. Luo, J. Hu, Y. Zhuang, X. Wei, X. Huang, J. Mol. Model. 2013, 19, 3333.

[17] L. Li, J. Hu, X. Shi, W. Ruan, J. Luo, X. Wei, Int. J. Mol. Sci. 2016, 17, 927.

[18] S. Grimme, S. Ehrlich, L. Goerigk, J. Comp. Chem. 2011, 32, 1456.

[19] B. Y. Simkin, I. I. Sheikhet, Quantum Chemical and Statistical Theory of Solutions: A Computational Approach, Ellis Horwood, London, UK 1995.

[20] E. Cances, B. Mennucci, J. Tomasi, J. Chem. Phys. 1997, 107, 3032.

[21] M. Cossi, V. Barone, R. Cammi, J. Tomasi, J. Chem. Phys. Lett. 1996, 255, 327.

[22] V. Barone, M. Cossi, J. Tomasi, J. Comput. Chem. 1998, 19, 404.

[23] M. J. Frisch, G. W. Trucks, H. B. Schlegel, G. E. Scuseria, M. A. Robb, J. R. Cheeseman, G. Scalmani, V. Barone, B. Mennucci, G. A. Petersson, H. Nakatsuji, M. Caricato, X. Li, H. P. Hratchian, A. F. Izmaylov, J. Bloino, G. Zheng, J. L. Sonnenberg, M. Hada, M. Ehara, K. Toyota, R. Fukuda, J. Hasegawa, M. Ishida, T. Nakajima, Y. Honda, O. Kitao, H. Nakai, T. Vreven, J. A. Montgomery Jr., J. E. Peralta, F. Ogliaro, M. Bearpark, J. J. Heyd, E. Brothers, K. N. Kudin, V. N. Staroverov, R. Kobayashi, J. 
Normand, K. Raghavachari, A. Rendell, J. C. Burant, S. S. Iyengar, J. Tomasi, M. Cossi, N. Rega, J. M. Millam, M. Klene, J. E. Knox, J. B. Cross, V. Bakken, C. Adamo, J. Jaramillo, R. Gomperts, R. E. Stratmann, O. Yazyev, A. J. Austin, R. Cammi, C. Pomelli, J. W. Ochterski, R. L. Martin, K. Morokuma, V. G. Zakrzewski, G. A. Voth, P. Salvador, J. J. Dannenberg, S. Dapprich, A. D. Daniels, O. Farkas, J. B. Foresman, J. V. Ortiz, J. Cioslowski, D. J. Fox, Gaussian 09, Gaussian, Inc., Wallingford, CT 2009.

[24] R. F. W. Bader, Chem. Rev. 1991, 91, 893.

[25] I. Szabó, G. Czakó, Nat. Commun. 2015, 6, 5972.

[26] I. Szabó, G. Czakó, J. Phys. Chem. A 2017, 121(47), 9005.

[27] I. Szabó, G. Czakó, J. Phys. Chem. A 2017, 121(30), 5748.

[28] D. A. Tasi, Z. Fábián, G. Czakó, Phys. Chem. Chem. Phys. 2019, 21, 7924.

[29] M. N. Glukhovtsev, A. Pross, H. B. Schlegel, R. D. Bach, L. Radom, J. Am. Chem. Soc. 1996, 118, 11258. 


\section{Supporting Information}

Scheme 1S. Formation of the intermediate 12c (GD3BJ calculations). $G_{\text {rel }}-$ relative Gibbs free energies in $\mathrm{kcal} / \mathrm{mol}$. The sum of total free energies for the isolated reactants was assumed as a reference.
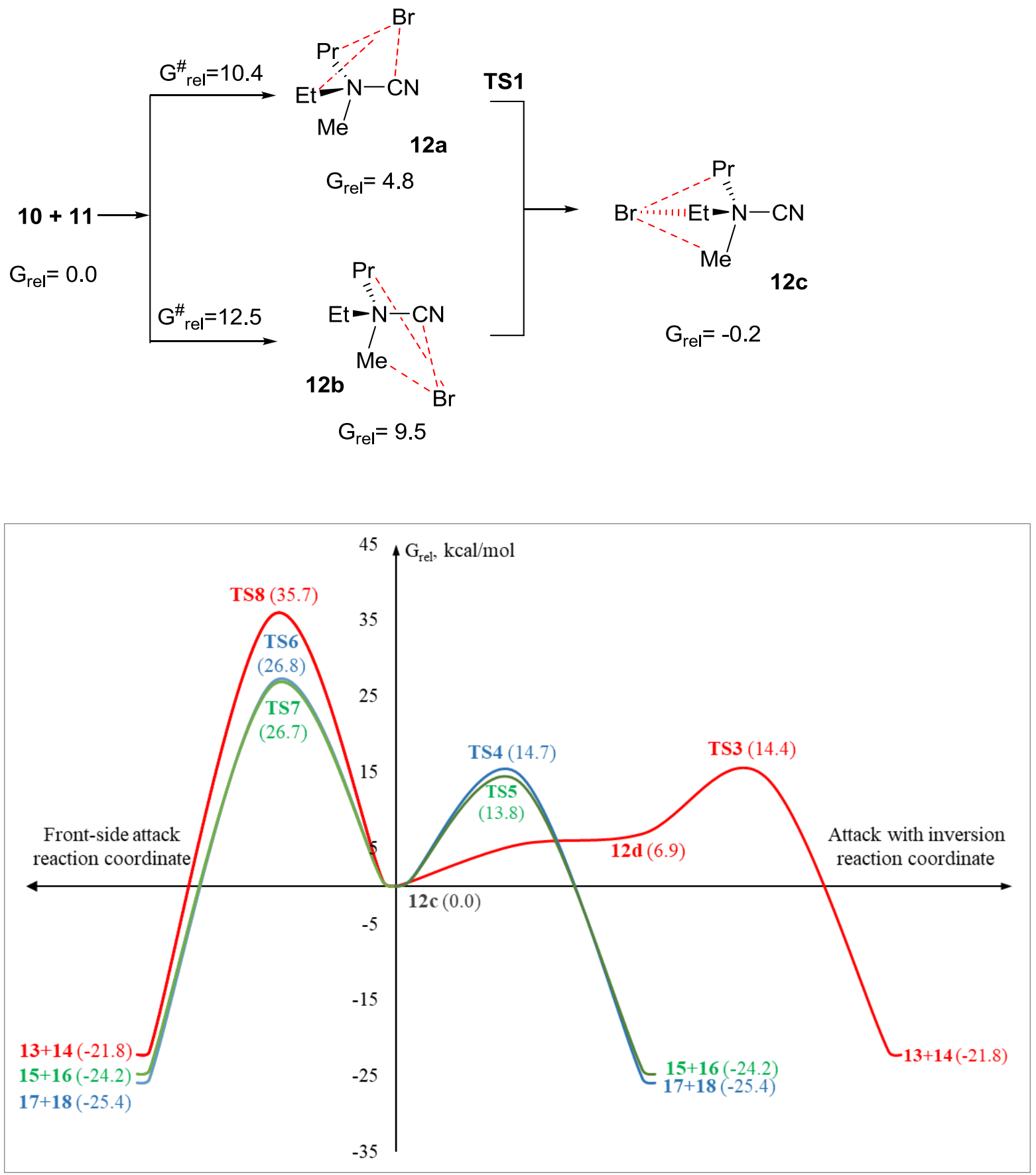

Figure 1S. MEPs for the von Braun reactions. PCM/DFT/B3LYP/6-31++G(d,p) including empirical dispersion (GD3BJ) calculations in $\mathrm{CHCl}_{3}, 100^{\circ} \mathrm{C}$. $\mathrm{G}_{\text {rel }}$ - relative Gibbs free energies. The total free energy of $\mathbf{1 2 c}$ is assumed as reference. The temperature was chosen according to experimental data. $^{[9]}$ 
Table S1. Optimized stationary point geometries shown in Scheme 4. PCM/B3LYP/6-31++G(d,p) calculations $\left(\right.$ Chloroform, $\left.120^{\circ} \mathrm{C}\right)$. Coordinates in angstroms. Sum of electronic and thermal Free Energies are in parentheses.

\begin{tabular}{|c|c|c|c|c|c|c|c|}
\hline \multicolumn{4}{|c|}{$10(-292.29153)$} & \multicolumn{4}{|c|}{ 12a $(-2956.83918)$} \\
\hline $\mathrm{N}$ & -0.558612000000 & 0.135324000000 & -0.168430000000 & $\mathrm{~N}$ & 1.378431000000 & -0.355825000000 & -0.113515000000 \\
\hline $\mathrm{C}$ & -1.650187000000 & -0.719797000000 & 0.319878000000 & $\mathrm{C}$ & 0.744154000000 & -1.480286000000 & -1.001016000000 \\
\hline $\mathrm{H}$ & -1.769656000000 & -0.619073000000 & 1.418310000000 & $\mathrm{H}$ & 1.107436000000 & -1.246747000000 & -2.003033000000 \\
\hline $\mathrm{H}$ & -1.356563000000 & -1.758214000000 & 0.131243000000 & $\mathrm{H}$ & -0.331541000000 & -1.291396000000 & -0.933116000000 \\
\hline $\mathrm{C}$ & 0.742346000000 & -0.360885000000 & 0.301055000000 & $\mathrm{C}$ & 0.872090000000 & 1.020746000000 & -0.656032000000 \\
\hline $\mathrm{H}$ & 0.856654000000 & -0.201474000000 & 1.393840000000 & $\mathrm{H}$ & 1.303580000000 & 1.077774000000 & -1.657621000000 \\
\hline $\mathrm{H}$ & 0.754192000000 & -1.445705000000 & 0.141570000000 & $\mathrm{H}$ & -0.214204000000 & 0.899766000000 & -0.711197000000 \\
\hline $\mathrm{C}$ & -2.994737000000 & -0.463884000000 & -0.363996000000 & $\mathrm{C}$ & 1.102667000000 & -2.887113000000 & -0.564340000000 \\
\hline $\mathrm{H}$ & -3.408166000000 & 0.520484000000 & -0.124853000000 & $\mathrm{H}$ & 2.158569000000 & -3.132218000000 & -0.700063000000 \\
\hline $\mathrm{H}$ & -2.895192000000 & -0.539346000000 & -1.452059000000 & $\mathrm{H}$ & 0.810486000000 & -3.084279000000 & 0.470771000000 \\
\hline $\mathrm{H}$ & -3.723186000000 & -1.212011000000 & -0.033083000000 & $\mathrm{H}$ & 0.522775000000 & -3.566333000000 & -1.196492000000 \\
\hline $\mathrm{C}$ & -0.766772000000 & 1.535802000000 & 0.199221000000 & $\mathrm{C}$ & 2.900948000000 & -0.431564000000 & -0.148866000000 \\
\hline $\mathrm{H}$ & 0.026777000000 & 2.160477000000 & -0.215947000000 & $\mathrm{H}$ & 3.305475000000 & 0.392079000000 & 0.434157000000 \\
\hline $\mathrm{H}$ & -1.713225000000 & 1.899381000000 & -0.206467000000 & $\mathrm{H}$ & 3.212869000000 & -1.382627000000 & 0.276043000000 \\
\hline $\mathrm{H}$ & -0.782588000000 & 1.682483000000 & 1.297986000000 & $\mathrm{H}$ & 3.199616000000 & -0.353376000000 & -1.193657000000 \\
\hline $\mathrm{C}$ & 1.950185000000 & 0.241304000000 & -0.426431000000 & $\mathrm{C}$ & 1.248499000000 & 2.216646000000 & 0.206653000000 \\
\hline $\mathrm{H}$ & 1.805985000000 & 0.116239000000 & -1.506914000000 & $\mathrm{H}$ & 0.912454000000 & 2.057987000000 & 1.237100000000 \\
\hline $\mathrm{H}$ & 2.013613000000 & 1.319505000000 & -0.239205000000 & $\mathrm{H}$ & 2.332115000000 & 2.370440000000 & 0.232083000000 \\
\hline $\mathrm{C}$ & 3.266055000000 & -0.417257000000 & 0.006727000000 & $\mathrm{C}$ & 0.565871000000 & 3.475665000000 & -0.352600000000 \\
\hline $\mathrm{H}$ & 3.262979000000 & -1.492347000000 & -0.208792000000 & $\mathrm{H}$ & -0.522707000000 & 3.370264000000 & -0.331437000000 \\
\hline $\mathrm{H}$ & 3.437999000000 & -0.294375000000 & 1.082673000000 & $\mathrm{H}$ & 0.872468000000 & 3.673987000000 & -1.385091000000 \\
\hline \multirow[t]{4}{*}{$\mathrm{H}$} & 4.119321000000 & 0.025009000000 & -0.518016000000 & $\mathrm{H}$ & 0.836248000000 & 4.345891000000 & 0.251475000000 \\
\hline & & & & $\mathrm{Br}$ & -2.427679000000 & -0.145643000000 & -0.018515000000 \\
\hline & & & & $\mathrm{C}$ & 0.973795000000 & -0.526073000000 & 1.219984000000 \\
\hline & & & & $\mathrm{N}$ & 0.766568000000 & -0.695866000000 & 2.347142000000 \\
\hline \multicolumn{4}{|c|}{ 12b $(-2956.82805)$} & \multicolumn{4}{|c|}{ 12c $(-2956.84665)$} \\
\hline $\mathrm{N}$ & 0.536971000000 & 0.798169000000 & -0.201082000000 & $\mathrm{~N}$ & -1.148932000000 & -0.460015000000 & 0.101999000000 \\
\hline $\mathrm{C}$ & 0.376065000000 & 2.300503000000 & 0.103156000000 & $\mathrm{C}$ & -0.504490000000 & -1.514930000000 & -0.863750000000 \\
\hline $\mathrm{H}$ & 1.009354000000 & 2.813833000000 & -0.624394000000 & $\mathrm{H}$ & 0.560665000000 & -1.263440000000 & -0.823931000000 \\
\hline $\mathrm{H}$ & 0.793455000000 & 2.431147000000 & 1.100965000000 & $\mathrm{H}$ & -0.913606000000 & -1.270370000000 & -1.845053000000 \\
\hline $\mathrm{C}$ & 2.021627000000 & 0.433399000000 & -0.019749000000 & $\mathrm{C}$ & -0.820611000000 & 0.960389000000 & -0.467470000000 \\
\hline $\mathrm{H}$ & 2.556056000000 & 1.014807000000 & -0.775611000000 & $\mathrm{H}$ & 0.273503000000 & 0.997039000000 & -0.426637000000 \\
\hline $\mathrm{H}$ & 2.290793000000 & 0.798773000000 & 0.971597000000 & $\mathrm{H}$ & -1.151575000000 & 0.922326000000 & -1.507282000000 \\
\hline $\mathrm{C}$ & -1.062370000000 & 2.794100000000 & 0.049820000000 & $\mathrm{C}$ & -0.783383000000 & -2.955327000000 & -0.480537000000 \\
\hline $\mathrm{H}$ & -1.481659000000 & 2.770956000000 & -0.958590000000 & $\mathrm{H}$ & -0.294977000000 & -3.247430000000 & 0.451177000000 \\
\hline $\mathrm{H}$ & -1.709952000000 & 2.227842000000 & 0.722513000000 & $\mathrm{H}$ & -1.852323000000 & -3.180120000000 & -0.421540000000 \\
\hline $\mathrm{H}$ & -1.062204000000 & 3.837411000000 & 0.379780000000 & $\mathrm{H}$ & -0.361001000000 & -3.576604000000 & -1.276051000000 \\
\hline $\mathrm{C}$ & 0.141435000000 & 0.540143000000 & -1.632039000000 & $\mathrm{C}$ & -0.586318000000 & -0.614486000000 & 1.519053000000 \\
\hline $\mathrm{H}$ & 0.238754000000 & -0.516727000000 & -1.854160000000 & $\mathrm{H}$ & -1.045740000000 & 0.139264000000 & 2.153324000000 \\
\hline $\mathrm{H}$ & -0.887911000000 & 0.842078000000 & -1.788019000000 & $\mathrm{H}$ & -0.830461000000 & -1.610897000000 & 1.878510000000 \\
\hline $\mathrm{H}$ & 0.817163000000 & 1.131333000000 & -2.252102000000 & $\mathrm{H}$ & 0.493719000000 & -0.466559000000 & 1.425928000000 \\
\hline $\mathrm{C}$ & 2.326964000000 & -1.057135000000 & -0.141933000000 & $\mathrm{C}$ & -1.477199000000 & 2.113407000000 & 0.277969000000 \\
\hline $\mathrm{H}$ & 1.768631000000 & -1.610688000000 & 0.617609000000 & $\mathrm{H}$ & -2.562644000000 & 1.972728000000 & 0.335562000000 \\
\hline $\mathrm{H}$ & 2.016980000000 & -1.438828000000 & -1.119539000000 & $\mathrm{H}$ & -1.094130000000 & 2.181973000000 & 1.300384000000 \\
\hline $\mathrm{C}$ & 3.832215000000 & -1.302811000000 & 0.033004000000 & $\mathrm{C}$ & -1.170321000000 & 3.425844000000 & -0.462325000000 \\
\hline $\mathrm{H}$ & 4.182008000000 & -0.962461000000 & 1.013642000000 & $\mathrm{H}$ & -1.580716000000 & 3.416917000000 & -1.477393000000 \\
\hline $\mathrm{H}$ & 4.416389000000 & -0.783681000000 & -0.734989000000 & $\mathrm{H}$ & -0.091587000000 & 3.597711000000 & -0.530356000000 \\
\hline $\mathrm{H}$ & 4.050561000000 & -2.371425000000 & -0.046517000000 & $\mathrm{H}$ & -1.613990000000 & 4.269321000000 & 0.073150000000 \\
\hline $\mathrm{Br}$ & -1.911427000000 & -1.110938000000 & -0.095989000000 & $\mathrm{Br}$ & 2.661049000000 & 0.040218000000 & 0.031322000000 \\
\hline $\mathrm{C}$ & -0.213347000000 & 0.070685000000 & 0.886493000000 & $\mathrm{C}$ & -2.534152000000 & -0.647610000000 & 0.127426000000 \\
\hline $\mathrm{N}$ & -0.056114000000 & 0.062565000000 & 2.063217000000 & $\mathrm{~N}$ & -3.681497000000 & -0.810442000000 & 0.139678000000 \\
\hline \multicolumn{4}{|c|}{$11(-2664.56858)$} & & & & \\
\hline $\mathrm{Br}$ & 0.000000000000 & 0.651724000000 & 0.000000000000 & & & & \\
\hline $\mathrm{C}$ & 0.008648000000 & -1.127893000000 & 0.000000000000 & & & & \\
\hline $\mathrm{N}$ & -0.007413000000 & -2.291856000000 & 0.000000000000 & & & & \\
\hline
\end{tabular}


Table S2. Optimized stationary point geometries shown in Scheme 6. PCM/B3LYP/6-31++G(d,p) calculations $\left(\right.$ Chloroform, $\left.120^{\circ} \mathrm{C}\right)$. Coordinates in angstroms. Sum of electronic and thermal Free Energies are in parentheses.

\begin{tabular}{|c|c|c|c|c|c|c|c|}
\hline \multicolumn{4}{|c|}{ 12d (-2956.83657) } & \multicolumn{4}{|c|}{ TS2 (-2956.83435) } \\
\hline $\mathrm{N}$ & 1.324647000000 & 0.559761000000 & -0.120604000000 & $\mathrm{~N}$ & -1.460687000000 & -0.393024000000 & -0.090792000000 \\
\hline $\mathrm{C}$ & 1.519284000000 & 1.757453000000 & -1.100381000000 & $\mathrm{C}$ & -1.901560000000 & -1.439193000000 & -1.160923000000 \\
\hline $\mathrm{H}$ & 1.094314000000 & 1.398496000000 & -2.038784000000 & $\mathrm{H}$ & -1.171650000000 & -1.311323000000 & -1.961653000000 \\
\hline $\mathrm{H}$ & 2.600336000000 & 1.858779000000 & -1.206877000000 & $\mathrm{H}$ & -2.879234000000 & -1.095844000000 & -1.502641000000 \\
\hline $\mathrm{C}$ & 2.079950000000 & -0.671110000000 & -0.700721000000 & $\mathrm{C}$ & -1.629729000000 & 1.029135000000 & -0.709459000000 \\
\hline $\mathrm{H}$ & 1.606483000000 & -0.837019000000 & -1.670405000000 & $\mathrm{H}$ & -1.071648000000 & 0.974225000000 & -1.646067000000 \\
\hline $\mathrm{H}$ & 3.105045000000 & -0.328154000000 & -0.855081000000 & $\mathrm{H}$ & -2.695758000000 & 1.115776000000 & -0.929594000000 \\
\hline $\mathrm{C}$ & 0.874441000000 & 3.050479000000 & -0.635881000000 & $\mathrm{C}$ & -1.954322000000 & -2.868645000000 & -0.654657000000 \\
\hline $\mathrm{H}$ & -0.215161000000 & 2.998294000000 & -0.617532000000 & $\mathrm{H}$ & -0.973420000000 & -3.256853000000 & -0.374912000000 \\
\hline $\mathrm{H}$ & 1.243288000000 & 3.369047000000 & 0.342984000000 & $\mathrm{H}$ & -2.646809000000 & -2.986919000000 & 0.182877000000 \\
\hline $\mathrm{H}$ & 1.155521000000 & 3.821948000000 & -1.358862000000 & $\mathrm{H}$ & -2.327640000000 & -3.482006000000 & -1.480015000000 \\
\hline $\mathrm{C}$ & -0.179390000000 & 0.237771000000 & 0.057034000000 & $\mathrm{C}$ & 0.002100000000 & -0.627964000000 & 0.326460000000 \\
\hline $\mathrm{H}$ & -0.274535000000 & -0.587954000000 & 0.753206000000 & $\mathrm{H}$ & 0.254124000000 & 0.069628000000 & 1.118108000000 \\
\hline $\mathrm{H}$ & -0.678409000000 & 1.117962000000 & 0.445392000000 & $\mathrm{H}$ & 0.094876000000 & -1.650948000000 & 0.679761000000 \\
\hline $\mathrm{H}$ & -0.564943000000 & -0.029957000000 & -0.922499000000 & $\mathrm{H}$ & 0.630497000000 & -0.453856000000 & -0.543533000000 \\
\hline $\mathrm{C}$ & 2.032979000000 & -1.913380000000 & 0.180946000000 & $\mathrm{C}$ & -1.140187000000 & 2.167290000000 & 0.176302000000 \\
\hline $\mathrm{H}$ & 2.339788000000 & -1.664220000000 & 1.202954000000 & $\mathrm{H}$ & -1.590081000000 & 2.096556000000 & 1.173351000000 \\
\hline $\mathrm{H}$ & 1.016641000000 & -2.313170000000 & 0.226089000000 & $\mathrm{H}$ & -0.054392000000 & 2.116806000000 & 0.291553000000 \\
\hline $\mathrm{C}$ & 2.977960000000 & -2.983632000000 & -0.386806000000 & $\mathrm{C}$ & -1.519779000000 & 3.513776000000 & -0.460391000000 \\
\hline $\mathrm{H}$ & 4.017248000000 & -2.639193000000 & -0.388534000000 & $\mathrm{H}$ & -2.605676000000 & 3.627432000000 & -0.542013000000 \\
\hline $\mathrm{H}$ & 2.704515000000 & -3.256367000000 & -1.411319000000 & $\mathrm{H}$ & -1.085712000000 & 3.619756000000 & -1.459901000000 \\
\hline $\mathrm{H}$ & 2.923002000000 & -3.887787000000 & 0.224743000000 & $\mathrm{H}$ & -1.139657000000 & 4.332309000000 & 0.156061000000 \\
\hline $\mathrm{Br}$ & -3.282340000000 & -0.384486000000 & 0.055673000000 & $\mathrm{Br}$ & 3.226700000000 & -0.125517000000 & 0.071039000000 \\
\hline $\mathrm{C}$ & 1.875579000000 & 0.896255000000 & 1.120121000000 & $\mathrm{C}$ & -2.286790000000 & -0.504807000000 & 1.032476000000 \\
\hline $\mathrm{N}$ & 2.350199000000 & 1.182137000000 & 2.137761000000 & $\mathrm{~N}$ & -2.980844000000 & -0.598289000000 & 1.955565000000 \\
\hline \multicolumn{4}{|c|}{$13(-345.25763)$} & \multicolumn{4}{|c|}{ TS3 (-2956.82697) } \\
\hline $\mathrm{N}$ & -0.487381000000 & -0.135452000000 & 0.031105000000 & $\mathrm{~N}$ & -1.347851000000 & -0.548380000000 & -0.149304000000 \\
\hline $\mathrm{C}$ & -1.602294000000 & -1.060075000000 & -0.300130000000 & $\mathrm{C}$ & -1.518612000000 & -1.699438000000 & -1.138356000000 \\
\hline $\mathrm{H}$ & -1.276454000000 & -2.047872000000 & 0.036914000000 & $\mathrm{H}$ & -0.870084000000 & -1.439816000000 & -1.977846000000 \\
\hline $\mathrm{H}$ & -1.734390000000 & -1.107403000000 & -1.390720000000 & $\mathrm{H}$ & -2.556933000000 & -1.670104000000 & -1.480257000000 \\
\hline $\mathrm{C}$ & 0.877870000000 & -0.600073000000 & -0.320214000000 & $\mathrm{C}$ & -2.012319000000 & 0.715768000000 & -0.682173000000 \\
\hline $\mathrm{H}$ & 0.919789000000 & -1.651771000000 & -0.021444000000 & $\mathrm{H}$ & -1.561269000000 & 0.878691000000 & -1.664211000000 \\
\hline $\mathrm{H}$ & 1.019278000000 & -0.558901000000 & -1.410726000000 & $\mathrm{H}$ & -3.071767000000 & 0.478983000000 & -0.825267000000 \\
\hline $\mathrm{C}$ & -2.909898000000 & -0.679192000000 & 0.384461000000 & $\mathrm{C}$ & -1.178498000000 & -3.068910000000 & -0.568756000000 \\
\hline $\mathrm{H}$ & -2.791860000000 & -0.658836000000 & 1.472120000000 & $\mathrm{H}$ & -0.160809000000 & -3.123172000000 & -0.173659000000 \\
\hline $\mathrm{H}$ & -3.270292000000 & 0.300035000000 & 0.055734000000 & $\mathrm{H}$ & -1.875875000000 & -3.366761000000 & 0.218091000000 \\
\hline $\mathrm{H}$ & -3.675748000000 & -1.419052000000 & 0.133017000000 & $\mathrm{H}$ & -1.256694000000 & -3.797042000000 & -1.380894000000 \\
\hline $\mathrm{C}$ & 1.980410000000 & 0.183437000000 & 0.391470000000 & $\mathrm{C}$ & 0.526840000000 & -0.205043000000 & -0.012776000000 \\
\hline $\mathrm{H}$ & 1.916755000000 & 1.243116000000 & 0.118285000000 & $\mathrm{H}$ & 0.517731000000 & 0.367657000000 & 0.899797000000 \\
\hline $\mathrm{H}$ & 1.816853000000 & 0.123180000000 & 1.474017000000 & $\mathrm{H}$ & 0.857669000000 & -1.228480000000 & 0.034889000000 \\
\hline $\mathrm{C}$ & 3.372019000000 & -0.353720000000 & 0.036628000000 & $\mathrm{H}$ & 0.603325000000 & 0.313849000000 & -0.954963000000 \\
\hline $\mathrm{H}$ & 3.564669000000 & -0.280924000000 & -1.039762000000 & $\mathrm{C}$ & -1.847344000000 & 1.940136000000 & 0.213753000000 \\
\hline $\mathrm{H}$ & 3.480714000000 & -1.404983000000 & 0.327168000000 & $\mathrm{H}$ & -2.194661000000 & 1.710150000000 & 1.227239000000 \\
\hline $\mathrm{H}$ & 4.149705000000 & 0.216988000000 & 0.553138000000 & $\mathrm{H}$ & -0.792249000000 & 2.220721000000 & 0.284444000000 \\
\hline $\mathrm{C}$ & -0.730594000000 & 1.169499000000 & -0.093831000000 & $\mathrm{C}$ & -2.654029000000 & 3.120144000000 & -0.345786000000 \\
\hline \multirow[t]{6}{*}{$\mathrm{N}$} & -0.947489000000 & 2.319335000000 & -0.159397000000 & $\mathrm{H}$ & -3.723680000000 & 2.888457000000 & -0.387274000000 \\
\hline & & & & $\mathrm{H}$ & -2.323837000000 & 3.387057000000 & -1.355510000000 \\
\hline & & & & $\mathrm{H}$ & -2.525028000000 & 3.999779000000 & 0.290637000000 \\
\hline & & & & $\mathrm{Br}$ & 3.097070000000 & 0.299067000000 & 0.045392000000 \\
\hline & & & & $\mathrm{C}$ & -1.804885000000 & -0.865832000000 & 1.104694000000 \\
\hline & & & & $\mathrm{N}$ & -2.156464000000 & -1.124228000000 & 2.182513000000 \\
\hline \multicolumn{4}{|c|}{ TS4 (-2956.82487) } & \multicolumn{4}{|c|}{ TS5 (-2956.82730) } \\
\hline $\mathrm{N}$ & -1.744291000000 & -0.154578000000 & 0.283623000000 & $\mathrm{~N}$ & 1.360110000000 & 0.653323000000 & 0.271466000000 \\
\hline $\mathrm{C}$ & -1.962801000000 & -1.379044000000 & -0.596644000000 & $\mathrm{C}$ & -0.651452000000 & 0.601223000000 & 0.128796000000 \\
\hline $\mathrm{H}$ & -1.314560000000 & -2.149009000000 & -0.173409000000 & $\mathrm{H}$ & -0.719011000000 & -0.031083000000 & 1.001322000000 \\
\hline $\mathrm{H}$ & -1.576565000000 & -1.109901000000 & -1.581828000000 & $\mathrm{H}$ & -0.617128000000 & 0.102537000000 & -0.827997000000 \\
\hline $\mathrm{C}$ & 0.190811000000 & 0.124297000000 & 0.239087000000 & $\mathrm{C}$ & 1.755459000000 & -0.796860000000 & 0.049478000000 \\
\hline $\mathrm{H}$ & 0.360442000000 & -0.663740000000 & 0.958921000000 & $\mathrm{H}$ & 1.250911000000 & -1.355542000000 & 0.841418000000 \\
\hline $\mathrm{H}$ & 0.285575000000 & -0.161897000000 & -0.798535000000 & $\mathrm{H}$ & 1.315632000000 & -1.090426000000 & -0.906750000000 \\
\hline $\mathrm{C}$ & -3.409766000000 & -1.842909000000 & -0.683043000000 & $\mathrm{C}$ & -1.069907000000 & 2.033995000000 & 0.196902000000 \\
\hline $\mathrm{H}$ & -3.797423000000 & -2.181895000000 & 0.280681000000 & $\mathrm{H}$ & -0.898010000000 & 2.470142000000 & 1.183411000000 \\
\hline $\mathrm{H}$ & -4.063899000000 & -1.059480000000 & -1.077010000000 & $\mathrm{H}$ & -0.577256000000 & 2.633893000000 & -0.573935000000 \\
\hline $\mathrm{H}$ & -3.450983000000 & -2.689704000000 & -1.374051000000 & $\mathrm{H}$ & -2.143975000000 & 2.072076000000 & 0.001447000000 \\
\hline $\mathrm{C}$ & -2.108531000000 & -0.377628000000 & 1.725828000000 & $\mathrm{C}$ & 1.708674000000 & 1.178718000000 & 1.632387000000 \\
\hline $\mathrm{H}$ & -1.759735000000 & 0.472588000000 & 2.311104000000 & $\mathrm{H}$ & 2.788509000000 & 1.165256000000 & 1.784082000000 \\
\hline $\mathrm{H}$ & -3.188272000000 & -0.482976000000 & 1.835883000000 & $\mathrm{H}$ & 1.340748000000 & 2.199543000000 & 1.721631000000 \\
\hline $\mathrm{H}$ & -1.605777000000 & -1.287146000000 & 2.054368000000 & $\mathrm{H}$ & 1.217080000000 & 0.539813000000 & 2.366411000000 \\
\hline
\end{tabular}




\begin{tabular}{|c|c|c|c|c|c|c|c|}
\hline $\mathrm{C}$ & 0.446270000000 & 1.555537000000 & 0.638618000000 & $\mathrm{C}$ & 3.259659000000 & -1.062538000000 & 0.052754000000 \\
\hline $\mathrm{H}$ & -0.418209000000 & 1.945166000000 & 1.193866000000 & $\mathrm{H}$ & 3.743219000000 & -0.451665000000 & -0.718411000000 \\
\hline $\mathrm{H}$ & 1.269054000000 & 1.544000000000 & 1.358459000000 & $\mathrm{H}$ & 3.695811000000 & -0.777606000000 & 1.015866000000 \\
\hline $\mathrm{C}$ & 0.775868000000 & 2.483322000000 & -0.536180000000 & $\mathrm{C}$ & 3.540699000000 & -2.547984000000 & -0.213796000000 \\
\hline $\mathrm{H}$ & -0.035587000000 & 2.509632000000 & -1.269843000000 & $\mathrm{H}$ & 3.140543000000 & -2.860977000000 & -1.183934000000 \\
\hline $\mathrm{H}$ & 1.687421000000 & 2.143837000000 & -1.035385000000 & $\mathrm{H}$ & 3.093454000000 & -3.183179000000 & 0.558414000000 \\
\hline $\mathrm{H}$ & 0.937194000000 & 3.504206000000 & -0.177837000000 & $\mathrm{H}$ & 4.618212000000 & -2.734568000000 & -0.219034000000 \\
\hline $\mathrm{Br}$ & 2.851645000000 & -0.616376000000 & -0.090403000000 & $\mathrm{Br}$ & -3.083525000000 & -0.673301000000 & -0.096492000000 \\
\hline $\mathrm{C}$ & -2.379466000000 & 0.947661000000 & -0.227563000000 & $\mathrm{C}$ & 1.810661000000 & 1.465047000000 & -0.731477000000 \\
\hline $\mathrm{N}$ & -2.891501000000 & 1.893444000000 & -0.669611000000 & $\mathrm{~N}$ & 2.147302000000 & 2.152067000000 & -1.608180000000 \\
\hline \multicolumn{4}{|c|}{$14(-2611.63762)$} & \multicolumn{4}{|c|}{$16(-2650.93631)$} \\
\hline $\mathrm{C}$ & 1.548279000000 & 0.000006000000 & -0.000002000000 & $\mathrm{C}$ & 1.044274000000 & 0.692043000000 & 0.000009000000 \\
\hline $\mathrm{H}$ & 1.868548000000 & -1.001829000000 & 0.275059000000 & $\mathrm{H}$ & 1.080796000000 & 1.314494000000 & 0.892929000000 \\
\hline $\mathrm{H}$ & 1.869078000000 & 0.262635000000 & -1.005005000000 & $\mathrm{H}$ & 1.080793000000 & 1.314522000000 & -0.892865000000 \\
\hline $\mathrm{H}$ & 1.868911000000 & 0.738984000000 & 0.730072000000 & $\mathrm{C}$ & 2.088166000000 & -0.404938000000 & 0.000025000000 \\
\hline \multirow[t]{4}{*}{$\mathrm{Br}$} & -0.425606000000 & 0.000005000000 & -0.000003000000 & $\mathrm{H}$ & 2.005309000000 & -1.035959000000 & 0.888822000000 \\
\hline & & & & $\mathrm{H}$ & 2.003143000000 & -1.038066000000 & -0.887085000000 \\
\hline & & & & $\mathrm{H}$ & 3.082572000000 & 0.058595000000 & -0.001918000000 \\
\hline & & & & $\mathrm{Br}$ & -0.801350000000 & -0.066749000000 & -0.000002000000 \\
\hline \multicolumn{4}{|c|}{$17(-266.67089)$} & \multicolumn{4}{|c|}{$18(-2690.23070)$} \\
\hline $\mathrm{N}$ & 0.225709000000 & 0.375440000000 & 0.337275000000 & $\mathrm{C}$ & 0.575104000000 & 0.622581000000 & -0.000013000000 \\
\hline $\mathrm{C}$ & -1.042011000000 & -0.302235000000 & 0.691255000000 & $\mathrm{H}$ & 0.642977000000 & 1.244511000000 & -0.892602000000 \\
\hline $\mathrm{H}$ & -1.605583000000 & 0.403476000000 & 1.310127000000 & $\mathrm{H}$ & 0.642959000000 & 1.244528000000 & 0.892567000000 \\
\hline $\mathrm{H}$ & -0.785346000000 & -1.156745000000 & 1.323020000000 & $\mathrm{C}$ & 1.586098000000 & -0.510093000000 & -0.000010000000 \\
\hline $\mathrm{C}$ & -1.872166000000 & -0.752235000000 & -0.512245000000 & $\mathrm{H}$ & 1.428530000000 & -1.142360000000 & 0.880568000000 \\
\hline $\mathrm{H}$ & -2.168270000000 & 0.094402000000 & -1.139269000000 & $\mathrm{H}$ & 1.428595000000 & -1.142237000000 & -0.880695000000 \\
\hline $\mathrm{H}$ & -1.315375000000 & -1.464197000000 & -1.129972000000 & $\mathrm{C}$ & 3.018334000000 & 0.045295000000 & 0.000019000000 \\
\hline $\mathrm{H}$ & -2.785160000000 & -1.246236000000 & -0.163830000000 & $\mathrm{H}$ & 3.208149000000 & 0.661001000000 & 0.886257000000 \\
\hline $\mathrm{C}$ & 0.162421000000 & 1.732793000000 & -0.227284000000 & $\mathrm{H}$ & 3.208081000000 & 0.661247000000 & -0.886059000000 \\
\hline $\mathrm{H}$ & 1.161962000000 & 2.170095000000 & -0.225208000000 & $\mathrm{H}$ & 3.744769000000 & -0.773574000000 & -0.000125000000 \\
\hline $\mathrm{H}$ & -0.228741000000 & 1.738191000000 & -1.251357000000 & $\mathrm{Br}$ & -1.296608000000 & -0.048566000000 & 0.000003000000 \\
\hline $\mathrm{H}$ & -0.487000000000 & 2.338797000000 & 0.409147000000 & & & & \\
\hline $\mathrm{C}$ & 1.273472000000 & -0.380487000000 & 0.030607000000 & & & & \\
\hline $\mathrm{N}$ & 2.214750000000 & -1.041839000000 & -0.198226000000 & & & & \\
\hline
\end{tabular}


Table S3. Optimized stationary point geometries shown in Scheme 8. PCM/B3LYP/6-31++G(d,p) calculations (Chloroform, $-25^{\circ} \mathrm{C}$ ). Coordinates in angstroms. Sum of electronic and thermal Free Energies are in parentheses.

\begin{tabular}{|c|c|c|c|c|c|c|c|}
\hline \multicolumn{4}{|c|}{$19(-597.25240)$} & \multicolumn{4}{|c|}{ 20a $(-3261.79341)$} \\
\hline $\mathrm{N}$ & 1.324647000000 & 0.559761000000 & -0.120604000000 & $\mathrm{C}$ & 0.293774000000 & 0.501886000000 & -1.457030000000 \\
\hline $\mathrm{C}$ & 1.519284000000 & 1.757453000000 & -1.100381000000 & $\mathrm{C}$ & -1.110332000000 & 0.909839000000 & -0.991826000000 \\
\hline $\mathrm{H}$ & 1.094314000000 & 1.398496000000 & -2.038784000000 & $\mathrm{C}$ & -1.221115000000 & -0.391293000000 & -0.170649000000 \\
\hline $\mathrm{H}$ & 2.600336000000 & 1.858779000000 & -1.206877000000 & $\mathrm{~N}$ & 0.361800000000 & -0.638259000000 & -0.396624000000 \\
\hline $\mathrm{C}$ & 2.079950000000 & -0.671110000000 & -0.700721000000 & $\mathrm{H}$ & -1.767262000000 & -1.206315000000 & -0.635396000000 \\
\hline $\mathrm{H}$ & 1.606483000000 & -0.837019000000 & -1.670405000000 & $\mathrm{H}$ & -1.432638000000 & -0.318321000000 & 0.890885000000 \\
\hline $\mathrm{H}$ & 3.105045000000 & -0.328154000000 & -0.855081000000 & $\mathrm{H}$ & -1.858588000000 & 0.989194000000 & -1.784424000000 \\
\hline $\mathrm{C}$ & 0.874441000000 & 3.050479000000 & -0.635881000000 & $\mathrm{H}$ & 0.344815000000 & 0.063864000000 & -2.452488000000 \\
\hline $\mathrm{H}$ & -0.215161000000 & 2.998294000000 & -0.617532000000 & $\mathrm{H}$ & 1.094791000000 & 1.220922000000 & -1.296537000000 \\
\hline $\mathrm{H}$ & 1.243288000000 & 3.369047000000 & 0.342984000000 & $\mathrm{C}$ & 1.219030000000 & -0.289497000000 & 0.890815000000 \\
\hline $\mathrm{H}$ & 1.155521000000 & 3.821948000000 & -1.358862000000 & $\mathrm{H}$ & 0.724487000000 & 0.625054000000 & 1.224150000000 \\
\hline $\mathrm{C}$ & -0.179390000000 & 0.237771000000 & 0.057034000000 & $\mathrm{C}$ & 1.033705000000 & -1.395796000000 & 1.922842000000 \\
\hline $\mathrm{H}$ & -0.274535000000 & -0.587954000000 & 0.753206000000 & $\mathrm{H}$ & -0.021167000000 & -1.575187000000 & 2.144802000000 \\
\hline $\mathrm{H}$ & -0.678409000000 & 1.117962000000 & 0.445392000000 & $\mathrm{H}$ & 1.492925000000 & -2.337111000000 & 1.611819000000 \\
\hline $\mathrm{H}$ & -0.564943000000 & -0.029957000000 & -0.922499000000 & $\mathrm{H}$ & 1.521557000000 & -1.073618000000 & 2.846192000000 \\
\hline $\mathrm{C}$ & 2.032979000000 & -1.913380000000 & 0.180946000000 & $\mathrm{C}$ & 2.649552000000 & 0.023681000000 & 0.516121000000 \\
\hline $\mathrm{H}$ & 2.339788000000 & -1.664220000000 & 1.202954000000 & $\mathrm{C}$ & 3.572711000000 & -0.979640000000 & 0.177881000000 \\
\hline $\mathrm{H}$ & 1.016641000000 & -2.313170000000 & 0.226089000000 & $\mathrm{C}$ & 3.074630000000 & 1.362047000000 & 0.534057000000 \\
\hline $\mathrm{C}$ & 2.977960000000 & -2.983632000000 & -0.386806000000 & $\mathrm{C}$ & 4.887651000000 & -0.645158000000 & -0.150075000000 \\
\hline $\mathrm{H}$ & 4.017248000000 & -2.639193000000 & -0.388534000000 & $\mathrm{C}$ & 4.393318000000 & 1.694374000000 & 0.215450000000 \\
\hline $\mathrm{H}$ & 2.704515000000 & -3.256367000000 & -1.411319000000 & $\mathrm{C}$ & 5.300577000000 & 0.690814000000 & -0.132860000000 \\
\hline $\mathrm{H}$ & 2.923002000000 & -3.887787000000 & 0.224743000000 & $\mathrm{H}$ & 3.279899000000 & -2.023979000000 & 0.173681000000 \\
\hline $\mathrm{Br}$ & -3.282340000000 & -0.384486000000 & 0.055673000000 & $\mathrm{H}$ & 2.375320000000 & 2.146475000000 & 0.811229000000 \\
\hline $\mathrm{C}$ & 1.875579000000 & 0.896255000000 & 1.120121000000 & $\mathrm{H}$ & 5.590706000000 & -1.429847000000 & -0.411379000000 \\
\hline \multirow[t]{10}{*}{$\mathrm{N}$} & 2.350199000000 & 1.182137000000 & 2.137761000000 & $\mathrm{H}$ & 4.708100000000 & 2.732929000000 & 0.240188000000 \\
\hline & & & & $\mathrm{H}$ & 6.325814000000 & 0.945975000000 & -0.382931000000 \\
\hline & & & & $\mathrm{O}$ & -1.025527000000 & 2.085572000000 & -0.226577000000 \\
\hline & & & & $\mathrm{C}$ & -2.276712000000 & 2.782585000000 & -0.066739000000 \\
\hline & & & & $\mathrm{H}$ & -2.095097000000 & 3.566550000000 & 0.669588000000 \\
\hline & & & & $\mathrm{H}$ & -2.575220000000 & 3.235745000000 & -1.020460000000 \\
\hline & & & & $\mathrm{H}$ & -3.062266000000 & 2.104447000000 & 0.279465000000 \\
\hline & & & & $\mathrm{Br}$ & -4.294716000000 & -0.394560000000 & 0.263113000000 \\
\hline & & & & $\mathrm{C}$ & 0.670796000000 & -1.878368000000 & -0.922870000000 \\
\hline & & & & $\mathrm{N}$ & 0.916425000000 & -2.912400000000 & -1.388438000000 \\
\hline \multicolumn{4}{|c|}{ 20b $(-3261.79378)$} & \multicolumn{4}{|c|}{ TS9 $(-3261.79411)$} \\
\hline $\mathrm{C}$ & -0.729334000000 & -0.472192000000 & -0.343507000000 & $\mathrm{C}$ & -0.186397000000 & 0.348374000000 & 1.438068000000 \\
\hline $\mathrm{C}$ & -1.447124000000 & -1.624709000000 & 0.383866000000 & $\mathrm{C}$ & 1.197672000000 & 0.791700000000 & 0.951849000000 \\
\hline $\mathrm{C}$ & -0.384170000000 & -2.601288000000 & -0.129440000000 & $\mathrm{C}$ & 1.398571000000 & -0.476295000000 & 0.108317000000 \\
\hline $\mathrm{N}$ & 0.561455000000 & -1.417137000000 & -0.512223000000 & $\mathrm{~N}$ & -0.366191000000 & -0.732792000000 & 0.358919000000 \\
\hline $\mathrm{H}$ & -0.655792000000 & -3.144155000000 & -1.032995000000 & $\mathrm{H}$ & 1.826396000000 & -1.351204000000 & 0.579271000000 \\
\hline $\mathrm{H}$ & 0.083220000000 & -3.251905000000 & 0.607698000000 & $\mathrm{H}$ & 1.510202000000 & -0.412740000000 & -0.965374000000 \\
\hline $\mathrm{H}$ & -2.468011000000 & -1.796677000000 & 0.033433000000 & $\mathrm{H}$ & 1.947811000000 & 0.896234000000 & 1.739742000000 \\
\hline $\mathrm{H}$ & -1.112229000000 & -0.239426000000 & -1.332883000000 & $\mathrm{H}$ & -0.179928000000 & -0.129488000000 & 2.416850000000 \\
\hline $\mathrm{H}$ & -0.469364000000 & 0.427408000000 & 0.204642000000 & $\mathrm{H}$ & -0.977924000000 & 1.093916000000 & 1.375831000000 \\
\hline $\mathrm{C}$ & 1.705655000000 & -1.149082000000 & 0.562159000000 & $\mathrm{C}$ & -1.200630000000 & -0.302019000000 & -0.882880000000 \\
\hline $\mathrm{H}$ & 1.142288000000 & -1.264558000000 & 1.489787000000 & $\mathrm{H}$ & -0.681647000000 & 0.605844000000 & -1.197485000000 \\
\hline $\mathrm{C}$ & 2.765799000000 & -2.236858000000 & 0.441785000000 & $\mathrm{C}$ & -1.077177000000 & -1.368557000000 & -1.967849000000 \\
\hline $\mathrm{H}$ & 2.336189000000 & -3.238302000000 & 0.526298000000 & $\mathrm{H}$ & -0.031714000000 & -1.573614000000 & -2.212904000000 \\
\hline $\mathrm{H}$ & 3.325460000000 & -2.176027000000 & -0.494332000000 & $\mathrm{H}$ & -1.559750000000 & -2.307189000000 & -1.684233000000 \\
\hline $\mathrm{H}$ & 3.473121000000 & -2.106092000000 & 1.264706000000 & $\mathrm{H}$ & -1.569589000000 & -0.995873000000 & -2.869278000000 \\
\hline $\mathrm{C}$ & 2.205051000000 & 0.273004000000 & 0.452113000000 & $\mathrm{C}$ & -2.620197000000 & 0.057528000000 & -0.490988000000 \\
\hline $\mathrm{C}$ & 3.146702000000 & 0.663036000000 & -0.514557000000 & $\mathrm{C}$ & -3.569934000000 & -0.918256000000 & -0.146101000000 \\
\hline $\mathrm{C}$ & 1.731522000000 & 1.229912000000 & 1.365332000000 & $\mathrm{C}$ & -3.003879000000 & 1.407576000000 & -0.493475000000 \\
\hline $\mathrm{C}$ & 3.583338000000 & 1.987447000000 & -0.577983000000 & $\mathrm{C}$ & -4.870569000000 & -0.545981000000 & 0.198580000000 \\
\hline $\mathrm{C}$ & 2.173961000000 & 2.552547000000 & 1.304513000000 & $\mathrm{C}$ & -4.308364000000 & 1.778855000000 & -0.157349000000 \\
\hline $\mathrm{C}$ & 3.095989000000 & 2.934854000000 & 0.327179000000 & $\mathrm{C}$ & -5.243068000000 & 0.802095000000 & 0.194026000000 \\
\hline $\mathrm{H}$ & 3.547373000000 & -0.055438000000 & -1.221422000000 & $\mathrm{H}$ & -3.307571000000 & -1.970891000000 & -0.147921000000 \\
\hline $\mathrm{H}$ & 1.017871000000 & 0.940031000000 & 2.131902000000 & $\mathrm{H}$ & -2.282313000000 & 2.171812000000 & -0.769930000000 \\
\hline $\mathrm{H}$ & 4.306460000000 & 2.277333000000 & -1.333847000000 & $\mathrm{H}$ & -5.594273000000 & -1.310519000000 & 0.463894000000 \\
\hline $\mathrm{H}$ & 1.797242000000 & 3.279575000000 & 2.016904000000 & $\mathrm{H}$ & -4.590267000000 & 2.827198000000 & -0.170140000000 \\
\hline $\mathrm{H}$ & 3.439979000000 & 3.963152000000 & 0.275852000000 & $\mathrm{H}$ & -6.257056000000 & 1.087213000000 & 0.457548000000 \\
\hline $\mathrm{O}$ & -1.368135000000 & -1.577912000000 & 1.788983000000 & $\mathrm{O}$ & 1.063892000000 & 1.971374000000 & 0.193271000000 \\
\hline $\mathrm{C}$ & -2.405548000000 & -0.807697000000 & 2.418841000000 & $\mathrm{C}$ & 2.244763000000 & 2.790457000000 & 0.118413000000 \\
\hline $\mathrm{H}$ & -2.203496000000 & -0.853528000000 & 3.489836000000 & $\mathrm{H}$ & 2.000530000000 & 3.607754000000 & -0.561748000000 \\
\hline $\mathrm{H}$ & -2.400418000000 & 0.229440000000 & 2.070849000000 & $\mathrm{H}$ & 2.484100000000 & 3.198043000000 & 1.108864000000 \\
\hline $\mathrm{H}$ & -3.387049000000 & -1.248416000000 & 2.207379000000 & $\mathrm{H}$ & 3.095039000000 & 2.217649000000 & -0.259997000000 \\
\hline $\mathrm{Br}$ & -3.259855000000 & 1.309140000000 & -0.708269000000 & $\mathrm{Br}$ & 4.175622000000 & -0.326460000000 & -0.243001000000 \\
\hline $\mathrm{C}$ & 1.060299000000 & -1.472671000000 & -1.800490000000 & $\mathrm{C}$ & -0.683276000000 & -1.973719000000 & 0.842190000000 \\
\hline $\mathrm{N}$ & 1.474879000000 & -1.525266000000 & -2.882792000000 & $\mathrm{~N}$ & -0.913100000000 & -3.031432000000 & 1.266664000000 \\
\hline
\end{tabular}




\begin{tabular}{|c|c|c|c|c|c|c|c|}
\hline \multicolumn{4}{|c|}{ TS10 (-3261.79325) } & \multicolumn{4}{|c|}{ 20a $(-3261.85494)$} \\
\hline $\mathrm{C}$ & 0.870979000000 & -0.326913000000 & 0.456010000000 & $\mathrm{C}$ & 0.118584000000 & 0.091604000000 & -1.127608000000 \\
\hline $\mathrm{C}$ & 1.584641000000 & -1.496890000000 & -0.237542000000 & $\mathrm{C}$ & 1.472997000000 & -0.516649000000 & -0.705036000000 \\
\hline $\mathrm{C}$ & 0.506485000000 & -2.504202000000 & 0.169050000000 & $\mathrm{C}$ & 2.401437000000 & 0.572848000000 & -0.198976000000 \\
\hline $\mathrm{N}$ & -0.554681000000 & -1.435865000000 & 0.492894000000 & $\mathrm{~N}$ & -0.496465000000 & 0.942834000000 & -0.091697000000 \\
\hline $\mathrm{H}$ & 0.739478000000 & -3.058356000000 & 1.077320000000 & $\mathrm{H}$ & 2.461249000000 & 1.407058000000 & -0.896494000000 \\
\hline $\mathrm{H}$ & 0.164181000000 & -3.174113000000 & -0.618750000000 & $\mathrm{H}$ & 2.092216000000 & 0.925778000000 & 0.782875000000 \\
\hline $\mathrm{H}$ & 2.578894000000 & -1.691767000000 & 0.172369000000 & $\mathrm{H}$ & 1.908979000000 & -0.989016000000 & -1.597408000000 \\
\hline $\mathrm{H}$ & 1.044745000000 & -0.164242000000 & 1.511181000000 & $\mathrm{H}$ & 0.257049000000 & 0.688650000000 & -2.033003000000 \\
\hline $\mathrm{H}$ & 0.532866000000 & 0.531942000000 & -0.107053000000 & $\mathrm{H}$ & -0.583483000000 & -0.711461000000 & -1.374047000000 \\
\hline $\mathrm{C}$ & -1.584110000000 & -1.169776000000 & -0.650993000000 & $\mathrm{C}$ & -1.391872000000 & 0.316384000000 & 0.923644000000 \\
\hline $\mathrm{H}$ & -0.942684000000 & -1.186290000000 & -1.534241000000 & $\mathrm{H}$ & -0.867962000000 & -0.593142000000 & 1.227946000000 \\
\hline $\mathrm{C}$ & -2.592135000000 & -2.315168000000 & -0.716591000000 & $\mathrm{C}$ & -1.533723000000 & 1.219550000000 & 2.154594000000 \\
\hline $\mathrm{H}$ & -2.098431000000 & -3.281759000000 & -0.846072000000 & $\mathrm{H}$ & -0.552888000000 & 1.441760000000 & 2.585869000000 \\
\hline $\mathrm{H}$ & -3.222791000000 & -2.364818000000 & 0.174382000000 & $\mathrm{H}$ & -2.033462000000 & 2.161281000000 & 1.908626000000 \\
\hline $\mathrm{H}$ & -3.241331000000 & -2.151877000000 & -1.580679000000 & $\mathrm{H}$ & -2.136866000000 & 0.705375000000 & 2.907189000000 \\
\hline $\mathrm{C}$ & -2.186008000000 & 0.214106000000 & -0.509192000000 & $\mathrm{C}$ & -2.743526000000 & -0.077671000000 & 0.334676000000 \\
\hline $\mathrm{C}$ & -3.229746000000 & 0.487542000000 & 0.389582000000 & $\mathrm{C}$ & -3.462475000000 & 0.785409000000 & -0.505531000000 \\
\hline $\mathrm{C}$ & -1.700117000000 & 1.252717000000 & -1.319685000000 & $\mathrm{C}$ & -3.308427000000 & -1.315008000000 & 0.673567000000 \\
\hline $\mathrm{C}$ & -3.754098000000 & 1.777838000000 & 0.490871000000 & $\mathrm{C}$ & -4.725202000000 & 0.423713000000 & -0.981523000000 \\
\hline $\mathrm{C}$ & -2.227373000000 & 2.542076000000 & -1.220603000000 & $\mathrm{C}$ & -4.571191000000 & -1.680341000000 & 0.196680000000 \\
\hline $\mathrm{C}$ & -3.250861000000 & 2.808706000000 & -0.307986000000 & $\mathrm{C}$ & -5.284060000000 & -0.810169000000 & -0.632454000000 \\
\hline $\mathrm{H}$ & -3.642640000000 & -0.296863000000 & 1.014739000000 & $\mathrm{H}$ & -3.043228000000 & 1.746287000000 & -0.789019000000 \\
\hline $\mathrm{H}$ & -0.909243000000 & 1.052551000000 & -2.037848000000 & $\mathrm{H}$ & -2.758466000000 & -1.997820000000 & 1.317094000000 \\
\hline $\mathrm{H}$ & -4.556334000000 & 1.976084000000 & 1.194989000000 & $\mathrm{H}$ & -5.271005000000 & 1.105431000000 & -1.627419000000 \\
\hline $\mathrm{H}$ & -1.837003000000 & 3.333365000000 & -1.852898000000 & $\mathrm{H}$ & -4.992817000000 & -2.643739000000 & 0.468637000000 \\
\hline $\mathrm{H}$ & -3.662497000000 & 3.810026000000 & -0.227836000000 & $\mathrm{H}$ & -6.264241000000 & -1.090864000000 & -1.006421000000 \\
\hline $\mathrm{O}$ & 1.603067000000 & -1.406068000000 & -1.644393000000 & $\mathrm{O}$ & 1.322171000000 & -1.483161000000 & 0.329130000000 \\
\hline $\mathrm{C}$ & 2.804587000000 & -0.857317000000 & -2.212719000000 & $\mathrm{C}$ & 1.106432000000 & -2.815442000000 & -0.129622000000 \\
\hline $\mathrm{H}$ & 2.644372000000 & -0.841017000000 & -3.291808000000 & $\mathrm{H}$ & 1.053172000000 & -3.446185000000 & 0.759590000000 \\
\hline $\mathrm{H}$ & 2.997171000000 & 0.149926000000 & -1.835130000000 & $\mathrm{H}$ & 0.166941000000 & -2.910560000000 & -0.690635000000 \\
\hline $\mathrm{H}$ & 3.660713000000 & -1.502904000000 & -1.979271000000 & $\mathrm{H}$ & 1.939229000000 & -3.150671000000 & -0.761493000000 \\
\hline $\mathrm{Br}$ & 3.066657000000 & 1.386758000000 & 0.702696000000 & $\mathrm{Br}$ & 4.250731000000 & -0.059061000000 & 0.026903000000 \\
\hline $\mathrm{C}$ & -1.129539000000 & -1.558505000000 & 1.728986000000 & $\mathrm{C}$ & -0.462820000000 & 2.258660000000 & -0.247548000000 \\
\hline $\mathrm{N}$ & -1.600066000000 & -1.643158000000 & 2.788857000000 & $\mathrm{~N}$ & -0.421289000000 & 3.423870000000 & -0.377688000000 \\
\hline \multicolumn{4}{|c|}{$20 b(-3261.85279)$} & \multicolumn{4}{|c|}{$11(-2664.55548)$} \\
\hline $\mathrm{C}$ & -1.481324000000 & -0.024714000000 & -0.779483000000 & $\mathrm{Br}$ & 0.000000000000 & 0.651724000000 & 0.000000000000 \\
\hline $\mathrm{C}$ & -1.536009000000 & -1.247521000000 & 0.119702000000 & $\mathrm{C}$ & 0.008648000000 & -1.127893000000 & 0.000000000000 \\
\hline $\mathrm{C}$ & -0.418698000000 & -2.244874000000 & -0.263747000000 & $\mathrm{~N}$ & -0.007413000000 & -2.291856000000 & 0.000000000000 \\
\hline $\mathrm{N}$ & 0.896779000000 & -1.623217000000 & -0.506348000000 & & & & \\
\hline $\mathrm{H}$ & -0.706643000000 & -2.772295000000 & -1.176501000000 & & & & \\
\hline $\mathrm{H}$ & -0.318756000000 & -2.994861000000 & 0.528964000000 & & & & \\
\hline $\mathrm{H}$ & -2.494802000000 & -1.768355000000 & -0.016272000000 & & & & \\
\hline $\mathrm{H}$ & -1.426203000000 & -0.299580000000 & -1.831951000000 & & & & \\
\hline $\mathrm{H}$ & -0.641494000000 & 0.614419000000 & -0.517521000000 & & & & \\
\hline $\mathrm{C}$ & 1.756571000000 & -1.168246000000 & 0.638454000000 & & & & \\
\hline $\mathrm{H}$ & 1.107685000000 & -1.285300000000 & 1.507735000000 & & & & \\
\hline $\mathrm{C}$ & 2.975326000000 & -2.083618000000 & 0.801543000000 & & & & \\
\hline $\mathrm{H}$ & 2.653961000000 & -3.118481000000 & 0.953351000000 & & & & \\
\hline $\mathrm{H}$ & 3.626768000000 & -2.056329000000 & -0.076848000000 & & & & \\
\hline $\mathrm{H}$ & 3.562594000000 & -1.770110000000 & 1.669623000000 & & & & \\
\hline $\mathrm{C}$ & 2.094999000000 & 0.313718000000 & 0.518563000000 & & & & \\
\hline $\mathrm{C}$ & 3.094657000000 & 0.775812000000 & -0.351356000000 & & & & \\
\hline $\mathrm{C}$ & 1.378561000000 & 1.250107000000 & 1.280034000000 & & & & \\
\hline $\mathrm{C}$ & 3.361328000000 & 2.142843000000 & -0.465061000000 & & & & \\
\hline $\mathrm{C}$ & 1.646535000000 & 2.617486000000 & 1.169112000000 & & & & \\
\hline $\mathrm{C}$ & 2.630499000000 & 3.068855000000 & 0.285254000000 & & & & \\
\hline $\mathrm{H}$ & 3.672160000000 & 0.075101000000 & -0.945792000000 & & & & \\
\hline $\mathrm{H}$ & 0.600036000000 & 0.904747000000 & 1.953868000000 & & & & \\
\hline $\mathrm{H}$ & 4.134777000000 & 2.483802000000 & -1.147240000000 & & & & \\
\hline $\mathrm{H}$ & 1.080176000000 & 3.327357000000 & 1.765049000000 & & & & \\
\hline $\mathrm{H}$ & 2.840209000000 & 4.130682000000 & 0.196103000000 & & & & \\
\hline $\mathrm{O}$ & -1.417932000000 & -0.811502000000 & 1.471623000000 & & & & \\
\hline $\mathrm{C}$ & -2.100799000000 & -1.628557000000 & 2.419073000000 & & & & \\
\hline $\mathrm{H}$ & -1.958190000000 & -1.160819000000 & 3.394923000000 & & & & \\
\hline $\mathrm{H}$ & -3.174034000000 & -1.675906000000 & 2.192696000000 & & & & \\
\hline $\mathrm{H}$ & -1.695030000000 & -2.648904000000 & 2.451613000000 & & & & \\
\hline $\mathrm{Br}$ & -3.072378000000 & 1.116817000000 & -0.607670000000 & & & & \\
\hline $\mathrm{C}$ & 1.399743000000 & -1.662833000000 & -1.731184000000 & & & & \\
\hline $\mathrm{N}$ & 1.846815000000 & -1.695714000000 & -2.815332000000 & & & & \\
\hline
\end{tabular}


Table S4. Optimized stationary point geometries shown in Scheme 9. PCM/B3LYP/6-31++G(d,p) calculations (Chloroform, $-25^{\circ} \mathrm{C}$ ). Coordinates in angstroms. Sum of electronic and thermal Free Energies are in parentheses.

\begin{tabular}{|c|c|c|c|c|c|c|c|}
\hline \multicolumn{4}{|c|}{ 20c $(-3261.80045)$} & \multicolumn{4}{|c|}{ TS11 $(-3261.76573)$} \\
\hline $\mathrm{C}$ & 0.838428000000 & 0.930504000000 & -0.946827000000 & $\mathrm{C}$ & 0.373469000000 & -1.393603000000 & 0.255668000000 \\
\hline $\mathrm{C}$ & 2.094864000000 & 1.677386000000 & -0.422919000000 & $\mathrm{C}$ & 1.783685000000 & -1.880984000000 & -0.116539000000 \\
\hline $\mathrm{C}$ & 1.549533000000 & 1.504862000000 & 1.000401000000 & $\mathrm{C}$ & 2.296079000000 & -0.726597000000 & -0.928972000000 \\
\hline $\mathrm{N}$ & 0.121017000000 & 1.259154000000 & 0.408421000000 & $\mathrm{~N}$ & 0.039884000000 & -0.468969000000 & -0.883486000000 \\
\hline $\mathrm{H}$ & 1.576024000000 & 2.331187000000 & 1.707627000000 & $\mathrm{H}$ & 2.256315000000 & -0.730222000000 & -2.012677000000 \\
\hline $\mathrm{H}$ & 1.869717000000 & 0.554571000000 & 1.432646000000 & $\mathrm{H}$ & 2.938333000000 & 0.002059000000 & -0.443594000000 \\
\hline $\mathrm{H}$ & 2.108361000000 & 2.727560000000 & -0.745488000000 & $\mathrm{H}$ & 1.748898000000 & -2.787472000000 & -0.741455000000 \\
\hline $\mathrm{H}$ & 0.279990000000 & 1.297756000000 & -1.805222000000 & $\mathrm{H}$ & -0.388097000000 & -2.161198000000 & 0.406337000000 \\
\hline $\mathrm{H}$ & 0.993346000000 & -0.152908000000 & -0.953291000000 & $\mathrm{H}$ & 0.463920000000 & -0.765642000000 & 1.143483000000 \\
\hline $\mathrm{C}$ & -0.763793000000 & 0.116095000000 & 1.081132000000 & $\mathrm{C}$ & -0.761726000000 & 0.777282000000 & -0.543552000000 \\
\hline $\mathrm{H}$ & -0.064793000000 & -0.728062000000 & 1.057572000000 & $\mathrm{H}$ & -0.133127000000 & 1.264018000000 & 0.205994000000 \\
\hline $\mathrm{C}$ & -1.054949000000 & 0.528981000000 & 2.515532000000 & $\mathrm{C}$ & -0.832891000000 & 1.697366000000 & -1.758507000000 \\
\hline $\mathrm{H}$ & -0.131165000000 & 0.679404000000 & 3.080661000000 & $\mathrm{H}$ & 0.174872000000 & 1.939932000000 & -2.104247000000 \\
\hline $\mathrm{H}$ & -1.666678000000 & 1.431637000000 & 2.595045000000 & $\mathrm{H}$ & -1.410503000000 & 1.270311000000 & -2.583548000000 \\
\hline $\mathrm{H}$ & -1.598457000000 & -0.291461000000 & 2.991435000000 & $\mathrm{H}$ & -1.321748000000 & 2.627215000000 & -1.455449000000 \\
\hline $\mathrm{C}$ & -1.948078000000 & -0.170189000000 & 0.187694000000 & $\mathrm{C}$ & -2.099928000000 & 0.408440000000 & 0.076945000000 \\
\hline $\mathrm{C}$ & -3.195415000000 & 0.449041000000 & 0.374158000000 & $\mathrm{C}$ & -3.145626000000 & -0.144529000000 & -0.681141000000 \\
\hline $\mathrm{C}$ & -1.807724000000 & -1.121996000000 & -0.837172000000 & $\mathrm{C}$ & -2.302484000000 & 0.633671000000 & 1.446853000000 \\
\hline $\mathrm{C}$ & -4.267964000000 & 0.149313000000 & -0.468176000000 & $\mathrm{C}$ & -4.363778000000 & -0.465393000000 & -0.078786000000 \\
\hline $\mathrm{C}$ & -2.884920000000 & -1.429709000000 & -1.670218000000 & $\mathrm{C}$ & -3.525135000000 & 0.322134000000 & 2.048253000000 \\
\hline $\mathrm{C}$ & -4.115388000000 & -0.791719000000 & -1.490769000000 & $\mathrm{C}$ & -4.556473000000 & -0.234379000000 & 1.287626000000 \\
\hline $\mathrm{H}$ & -3.340737000000 & 1.172035000000 & 1.169676000000 & $\mathrm{H}$ & -3.019226000000 & -0.319450000000 & -1.745275000000 \\
\hline $\mathrm{H}$ & -0.864641000000 & -1.649092000000 & -0.950258000000 & $\mathrm{H}$ & -1.505152000000 & 1.069666000000 & 2.042766000000 \\
\hline $\mathrm{H}$ & -5.224388000000 & 0.640090000000 & -0.316092000000 & $\mathrm{H}$ & -5.163420000000 & -0.891606000000 & -0.677269000000 \\
\hline $\mathrm{H}$ & -2.764022000000 & -2.174468000000 & -2.451051000000 & $\mathrm{H}$ & -3.668639000000 & 0.512138000000 & 3.107736000000 \\
\hline $\mathrm{H}$ & -4.953590000000 & -1.032201000000 & -2.138009000000 & $\mathrm{H}$ & -5.506592000000 & -0.480055000000 & 1.752603000000 \\
\hline $\mathrm{O}$ & 3.336807000000 & 1.077220000000 & -0.592359000000 & $\mathrm{O}$ & 2.647724000000 & -2.067778000000 & 0.978389000000 \\
\hline $\mathrm{C}$ & 3.886839000000 & 1.235995000000 & -1.904347000000 & $\mathrm{C}$ & 2.538803000000 & -3.350677000000 & 1.604740000000 \\
\hline $\mathrm{H}$ & 4.872954000000 & 0.772349000000 & -1.881422000000 & $\mathrm{H}$ & 3.315122000000 & -3.383019000000 & 2.369489000000 \\
\hline $\mathrm{H}$ & 3.269246000000 & 0.730043000000 & -2.656736000000 & $\mathrm{H}$ & 1.558693000000 & -3.483790000000 & 2.078617000000 \\
\hline $\mathrm{H}$ & 3.984016000000 & 2.298611000000 & -2.162491000000 & $\mathrm{H}$ & 2.706333000000 & -4.156047000000 & 0.878078000000 \\
\hline $\mathrm{Br}$ & 1.594837000000 & -2.382650000000 & 0.449030000000 & $\mathrm{Br}$ & 2.235336000000 & 2.127301000000 & 0.400262000000 \\
\hline $\mathrm{C}$ & -0.587229000000 & 2.451481000000 & 0.319189000000 & $\mathrm{C}$ & -0.286199000000 & -1.092174000000 & -2.036304000000 \\
\hline $\mathrm{N}$ & -1.167325000000 & 3.453371000000 & 0.251887000000 & $\mathrm{~N}$ & -0.492925000000 & -1.637245000000 & -3.047310000000 \\
\hline \multicolumn{4}{|c|}{ TS12 (-3261.79101) } & \multicolumn{4}{|c|}{ TS13 $(-3261.76543)$} \\
\hline
\end{tabular}




\begin{tabular}{|c|c|c|c|c|c|c|c|}
\hline $\mathrm{C}$ & -0.264230000000 & -2.018099000000 & -0.406622000000 & $\mathrm{C}$ & -1.606226000000 & -0.424198000000 & -1.273739000000 \\
\hline $\mathrm{C}$ & -1.810828000000 & -1.967369000000 & -0.545481000000 & $\mathrm{C}$ & -2.372647000000 & -1.340162000000 & -0.352721000000 \\
\hline $\mathrm{C}$ & -1.855036000000 & -1.035526000000 & 0.674983000000 & $\mathrm{C}$ & -1.486749000000 & -1.309146000000 & 0.894977000000 \\
\hline $\mathrm{N}$ & -0.371660000000 & -1.347602000000 & 0.961867000000 & $\mathrm{~N}$ & -0.111005000000 & -1.079051000000 & 0.323947000000 \\
\hline $\mathrm{H}$ & -2.508733000000 & -1.298351000000 & 1.505920000000 & $\mathrm{H}$ & -1.538639000000 & -2.195618000000 & 1.530445000000 \\
\hline $\mathrm{H}$ & -1.952089000000 & 0.021943000000 & 0.408255000000 & $\mathrm{H}$ & -1.746612000000 & -0.409220000000 & 1.455131000000 \\
\hline $\mathrm{H}$ & -2.274267000000 & -2.944922000000 & -0.357795000000 & $\mathrm{H}$ & -2.412164000000 & -2.349295000000 & -0.794609000000 \\
\hline $\mathrm{H}$ & 0.238179000000 & -2.984337000000 & -0.369220000000 & $\mathrm{H}$ & -0.905948000000 & -0.810651000000 & -2.007000000000 \\
\hline $\mathrm{H}$ & 0.243329000000 & -1.338336000000 & -1.093592000000 & $\mathrm{H}$ & -1.916743000000 & 0.614503000000 & -1.352741000000 \\
\hline $\mathrm{C}$ & 0.860420000000 & 0.411646000000 & 1.237696000000 & $\mathrm{C}$ & 0.855353000000 & -0.218153000000 & 1.124560000000 \\
\hline $\mathrm{H}$ & -0.017010000000 & 1.033317000000 & 1.043701000000 & $\mathrm{H}$ & 0.322947000000 & 0.736303000000 & 1.184811000000 \\
\hline $\mathrm{C}$ & 1.252191000000 & 0.296328000000 & 2.665955000000 & $\mathrm{C}$ & 1.033436000000 & -0.782463000000 & 2.536471000000 \\
\hline $\mathrm{H}$ & 0.383712000000 & 0.199915000000 & 3.319457000000 & $\mathrm{H}$ & 0.081092000000 & -0.807358000000 & 3.072558000000 \\
\hline $\mathrm{H}$ & 1.973308000000 & -0.493435000000 & 2.883415000000 & $\mathrm{H}$ & 1.456182000000 & -1.791454000000 & 2.538970000000 \\
\hline $\mathrm{H}$ & 1.726426000000 & 1.258414000000 & 2.912283000000 & $\mathrm{H}$ & 1.705591000000 & -0.124912000000 & 3.094749000000 \\
\hline $\mathrm{C}$ & 1.805063000000 & 0.335980000000 & 0.149202000000 & $\mathrm{C}$ & 2.129621000000 & -0.006800000000 & 0.323334000000 \\
\hline $\mathrm{C}$ & 3.085795000000 & -0.251968000000 & 0.288558000000 & $\mathrm{C}$ & 3.317227000000 & -0.696579000000 & 0.605207000000 \\
\hline $\mathrm{C}$ & 1.451553000000 & 0.936317000000 & -1.084082000000 & $\mathrm{C}$ & 2.120363000000 & 0.925502000000 & -0.727593000000 \\
\hline $\mathrm{C}$ & 3.973056000000 & -0.256817000000 & -0.780194000000 & $\mathrm{C}$ & 4.465975000000 & -0.478091000000 & -0.161055000000 \\
\hline $\mathrm{C}$ & 2.352227000000 & 0.938184000000 & -2.146622000000 & $\mathrm{C}$ & 3.267755000000 & 1.145057000000 & -1.491651000000 \\
\hline $\mathrm{C}$ & 3.608800000000 & 0.342973000000 & -1.995702000000 & $\mathrm{C}$ & 4.444373000000 & 0.442907000000 & -1.211122000000 \\
\hline $\mathrm{H}$ & 3.377375000000 & -0.714149000000 & 1.225588000000 & $\mathrm{H}$ & 3.357078000000 & -1.415092000000 & 1.417050000000 \\
\hline $\mathrm{H}$ & 0.481863000000 & 1.420881000000 & -1.172867000000 & $\mathrm{H}$ & 1.215379000000 & 1.492527000000 & -0.926618000000 \\
\hline $\mathrm{H}$ & 4.951670000000 & -0.713014000000 & -0.671707000000 & $\mathrm{H}$ & 5.376259000000 & -1.024406000000 & 0.067635000000 \\
\hline $\mathrm{H}$ & 2.079976000000 & 1.409439000000 & -3.085419000000 & $\mathrm{H}$ & 3.246161000000 & 1.873061000000 & -2.297407000000 \\
\hline $\mathrm{H}$ & 4.310385000000 & 0.345274000000 & -2.824632000000 & $\mathrm{H}$ & 5.337875000000 & 0.615448000000 & -1.803999000000 \\
\hline $\mathrm{O}$ & -2.332639000000 & -1.373801000000 & -1.698238000000 & $\mathrm{O}$ & -3.658530000000 & -0.891786000000 & -0.022671000000 \\
\hline $\mathrm{C}$ & -2.402369000000 & -2.256041000000 & -2.819869000000 & $\mathrm{C}$ & -4.651997000000 & -1.139379000000 & -1.025626000000 \\
\hline $\mathrm{H}$ & -2.867318000000 & -1.693484000000 & -3.630120000000 & $\mathrm{H}$ & -5.599128000000 & -0.800780000000 & -0.605533000000 \\
\hline $\mathrm{H}$ & -1.402609000000 & -2.582384000000 & -3.135927000000 & $\mathrm{H}$ & -4.445544000000 & -0.575754000000 & -1.943721000000 \\
\hline $\mathrm{H}$ & -3.014885000000 & -3.137291000000 & -2.587637000000 & $\mathrm{H}$ & -4.713357000000 & -2.210160000000 & -1.257526000000 \\
\hline $\mathrm{Br}$ & -1.623919000000 & 2.646123000000 & 0.240518000000 & $\mathrm{Br}$ & -1.321960000000 & 2.339920000000 & 0.243654000000 \\
\hline $\mathrm{C}$ & -0.133068000000 & -2.165699000000 & 2.014662000000 & $\mathrm{C}$ & 0.424778000000 & -2.167802000000 & -0.268862000000 \\
\hline $\mathrm{N}$ & 0.118449000000 & -2.834227000000 & 2.935732000000 & $\mathrm{~N}$ & 0.835126000000 & -3.104405000000 & -0.831345000000 \\
\hline \multicolumn{4}{|c|}{$21 \mathbf{a}^{`}(-3261.85721)$} & \multicolumn{4}{|c|}{ 21b` (-3261.85759) } \\
\hline $\mathrm{C}$ & -0.625418000000 & 0.950743000000 & 0.684123000000 & $\mathrm{C}$ & 1.689363000000 & -0.468175000000 & -0.976371000000 \\
\hline $\mathrm{C}$ & -2.121476000000 & 0.950324000000 & 0.310493000000 & $\mathrm{C}$ & 2.018102000000 & 0.970562000000 & -0.591125000000 \\
\hline $\mathrm{C}$ & -2.477888000000 & 0.014937000000 & -0.831674000000 & $\mathrm{C}$ & 1.142040000000 & 1.535036000000 & 0.538381000000 \\
\hline $\mathrm{N}$ & 0.238886000000 & 1.320132000000 & -0.453567000000 & $\mathrm{~N}$ & -0.298198000000 & 1.414778000000 & 0.247820000000 \\
\hline $\mathrm{H}$ & -1.937711000000 & 0.269784000000 & -1.741651000000 & $\mathrm{H}$ & 1.419428000000 & 2.580267000000 & 0.703655000000 \\
\hline $\mathrm{H}$ & -3.548441000000 & 0.035642000000 & -1.027003000000 & $\mathrm{H}$ & 1.346210000000 & 0.986082000000 & 1.459784000000 \\
\hline $\mathrm{H}$ & -2.393550000000 & 1.956525000000 & -0.051902000000 & $\mathrm{H}$ & 1.838232000000 & 1.559706000000 & -1.508923000000 \\
\hline $\mathrm{H}$ & -0.454341000000 & 1.641478000000 & 1.513344000000 & $\mathrm{H}$ & 0.639005000000 & -0.570197000000 & -1.244583000000 \\
\hline $\mathrm{H}$ & -0.331849000000 & -0.045249000000 & 1.023153000000 & $\mathrm{H}$ & 2.299303000000 & -0.815145000000 & -1.808084000000 \\
\hline $\mathrm{C}$ & 1.061060000000 & 0.257582000000 & -1.116164000000 & $\mathrm{C}$ & -1.187839000000 & 0.626390000000 & 1.169804000000 \\
\hline $\mathrm{H}$ & 0.370413000000 & -0.577844000000 & -1.255606000000 & $\mathrm{H}$ & -0.517932000000 & -0.129009000000 & 1.587300000000 \\
\hline $\mathrm{C}$ & 1.532513000000 & 0.715656000000 & -2.498144000000 & $\mathrm{C}$ & -1.711506000000 & 1.500576000000 & 2.314428000000 \\
\hline $\mathrm{H}$ & 0.682259000000 & 1.019494000000 & -3.116117000000 & $\mathrm{H}$ & -0.875519000000 & 1.924546000000 & 2.878788000000 \\
\hline $\mathrm{H}$ & 2.235023000000 & 1.551799000000 & -2.436418000000 & $\mathrm{H}$ & -2.324954000000 & 2.328161000000 & 1.946520000000 \\
\hline $\mathrm{H}$ & 2.042203000000 & -0.115140000000 & -2.992896000000 & $\mathrm{H}$ & -2.318969000000 & 0.900209000000 & 2.998313000000 \\
\hline $\mathrm{C}$ & 2.189988000000 & -0.224525000000 & -0.213338000000 & $\mathrm{C}$ & -2.261432000000 & -0.112397000000 & 0.381055000000 \\
\hline $\mathrm{C}$ & 3.221778000000 & 0.634837000000 & 0.196508000000 & $\mathrm{C}$ & -3.502087000000 & 0.465854000000 & 0.077669000000 \\
\hline $\mathrm{C}$ & 2.204480000000 & -1.555502000000 & 0.223898000000 & $\mathrm{C}$ & -1.998797000000 & -1.416232000000 & -0.068949000000 \\
\hline $\mathrm{C}$ & 4.247010000000 & 0.168187000000 & 1.021318000000 & $\mathrm{C}$ & -4.454135000000 & -0.239577000000 & -0.664924000000 \\
\hline $\mathrm{C}$ & 3.230308000000 & -2.024973000000 & 1.051199000000 & $\mathrm{C}$ & -2.948652000000 & -2.123370000000 & -0.809052000000 \\
\hline $\mathrm{C}$ & 4.256278000000 & -1.164941000000 & 1.448547000000 & $\mathrm{C}$ & -4.181493000000 & -1.535805000000 & -1.109400000000 \\
\hline $\mathrm{H}$ & 3.227408000000 & 1.672893000000 & -0.124453000000 & $\mathrm{H}$ & -3.736013000000 & 1.470426000000 & 0.414702000000 \\
\hline $\mathrm{H}$ & 1.406609000000 & -2.228475000000 & -0.079313000000 & $\mathrm{H}$ & -1.049110000000 & -1.886395000000 & 0.173202000000 \\
\hline $\mathrm{H}$ & 5.039504000000 & 0.843899000000 & 1.329764000000 & $\mathrm{H}$ & -5.409056000000 & 0.225174000000 & -0.892263000000 \\
\hline $\mathrm{H}$ & 3.225580000000 & -3.059324000000 & 1.382672000000 & $\mathrm{H}$ & -2.728969000000 & -3.132815000000 & -1.144655000000 \\
\hline $\mathrm{H}$ & 5.054777000000 & -1.526046000000 & 2.090002000000 & $\mathrm{H}$ & -4.923358000000 & -2.084097000000 & -1.682540000000 \\
\hline $\mathrm{O}$ & -2.909855000000 & 0.617780000000 & 1.443026000000 & $\mathrm{O}$ & 3.349983000000 & 1.169852000000 & -0.151655000000 \\
\hline $\mathrm{C}$ & -3.228425000000 & 1.716199000000 & 2.297498000000 & $\mathrm{C}$ & 4.362474000000 & 0.897923000000 & -1.118260000000 \\
\hline $\mathrm{H}$ & -3.860715000000 & 1.316126000000 & 3.091807000000 & $\mathrm{H}$ & 5.301926000000 & 1.246573000000 & -0.686285000000 \\
\hline $\mathrm{H}$ & -2.333724000000 & 2.163572000000 & 2.747989000000 & $\mathrm{H}$ & 4.446265000000 & -0.176067000000 & -1.326042000000 \\
\hline $\mathrm{H}$ & -3.781225000000 & 2.491497000000 & 1.750203000000 & $\mathrm{H}$ & 4.170596000000 & 1.438475000000 & -2.055128000000 \\
\hline $\mathrm{Br}$ & -2.054193000000 & -1.888443000000 & -0.465827000000 & $\mathrm{Br}$ & 2.000298000000 & -1.759013000000 & 0.488188000000 \\
\hline $\mathrm{C}$ & 0.502711000000 & 2.605160000000 & -0.646792000000 & $\mathrm{C}$ & -0.839097000000 & 2.326560000000 & -0.552878000000 \\
\hline $\mathrm{N}$ & 0.700523000000 & 3.748515000000 & -0.819098000000 & $\mathrm{~N}$ & -1.294521000000 & 3.124749000000 & -1.280586000000 \\
\hline \multicolumn{4}{|c|}{$22(-379.92708)$} & \multicolumn{4}{|c|}{$23(-2881.91932)$} \\
\hline
\end{tabular}




\begin{tabular}{|rrrrr|rrr|}
$\mathrm{C}$ & 0.069712000000 & -0.958002000000 & 0.216788000000 & $\mathrm{C}$ & 0.677239000000 & -0.931082000000 & -0.012323000000 \\
$\mathrm{C}$ & -0.870725000000 & 0.256326000000 & 0.490382000000 & $\mathrm{H}$ & 0.843759000000 & -1.594140000000 & -0.860129000000 \\
$\mathrm{C}$ & 0.300048000000 & 1.196595000000 & 0.128399000000 & $\mathrm{C}$ & 1.037132000000 & -1.617064000000 & 1.297322000000 \\
$\mathrm{~N}$ & 1.102960000000 & 0.002817000000 & -0.265605000000 & $\mathrm{H}$ & 2.043502000000 & -2.039818000000 & 1.252756000000 \\
$\mathrm{H}$ & 0.725672000000 & 1.746462000000 & 0.972599000000 & $\mathrm{H}$ & 0.986759000000 & -0.913896000000 & 2.132068000000 \\
$\mathrm{H}$ & 0.097927000000 & 1.870365000000 & -0.707495000000 & $\mathrm{H}$ & 0.326275000000 & -2.429403000000 & 1.489903000000 \\
$\mathrm{H}$ & -1.219548000000 & 0.328498000000 & 1.527549000000 & $\mathrm{C}$ & -0.708602000000 & -0.351556000000 & -0.050975000000 \\
$\mathrm{H}$ & 0.378655000000 & -1.510801000000 & 1.109065000000 & $\mathrm{C}$ & -1.135960000000 & 0.582722000000 & 0.905942000000 \\
$\mathrm{H}$ & -0.265702000000 & -1.646484000000 & -0.563163000000 & $\mathrm{C}$ & -1.641507000000 & -0.860709000000 & -0.964049000000 \\
$\mathrm{O}$ & -1.944118000000 & 0.415249000000 & -0.406346000000 & $\mathrm{C}$ & -2.459852000000 & 1.018797000000 & 0.927913000000 \\
$\mathrm{C}$ & -3.057015000000 & -0.427427000000 & -0.111731000000 & $\mathrm{C}$ & -2.968251000000 & -0.417122000000 & -0.950402000000 \\
$\mathrm{H}$ & -3.830202000000 & -0.196185000000 & -0.845877000000 & $\mathrm{C}$ & -3.380905000000 & 0.522054000000 & -0.002976000000 \\
$\mathrm{H}$ & -2.791036000000 & -1.489711000000 & -0.193172000000 & $\mathrm{H}$ & -0.425564000000 & 0.980359000000 & 1.624856000000 \\
$\mathrm{H}$ & -3.441428000000 & -0.229689000000 & 0.898261000000 & $\mathrm{H}$ & -1.328324000000 & -1.599714000000 & -1.697090000000 \\
$\mathrm{C}$ & 2.413448000000 & -0.134280000000 & -0.144529000000 & $\mathrm{H}$ & -2.775134000000 & 1.747216000000 & 1.669327000000 \\
$\mathrm{~N}$ & 3.577868000000 & -0.259062000000 & -0.080516000000 & $\mathrm{H}$ & -3.676527000000 & -0.813322000000 & -1.672148000000 \\
& & & & $\mathrm{H}$ & -4.410781000000 & 0.866390000000 & 0.012404000000 \\
& & & & $\mathrm{Br}$ & 2.054294000000 & 0.517717000000 & -0.310133000000 \\
\hline
\end{tabular}


Table S5. Optimized stationary point geometries shown in Scheme 10. PCM/B3LYP/6-31++G(d,p) calculations (Chloroform, $-25^{\circ} \mathrm{C}$ ). Coordinates in angstroms. Sum of electronic and thermal Free Energies are in parentheses.

\begin{tabular}{|c|c|c|c|c|c|c|c|}
\hline \multicolumn{4}{|c|}{$8(-828.23500)$} & \multicolumn{4}{|c|}{ 9a $(-3492.84167)$} \\
\hline $\mathrm{C}$ & 0.113852000000 & -1.528543000000 & -0.942243000000 & $\mathrm{C}$ & 0.523385000000 & -0.914759000000 & 0.609553000000 \\
\hline $\mathrm{C}$ & -0.203946000000 & -2.422437000000 & 0.284794000000 & $\mathrm{C}$ & 0.185034000000 & -1.489154000000 & -0.762473000000 \\
\hline $\mathrm{C}$ & 1.257863000000 & -2.159749000000 & 0.726852000000 & $\mathrm{C}$ & -1.081998000000 & -0.818550000000 & -1.342507000000 \\
\hline $\mathrm{N}$ & 1.545662000000 & -1.811352000000 & -0.686403000000 & $\mathrm{~N}$ & -2.285684000000 & -1.185046000000 & -0.569064000000 \\
\hline $\mathrm{H}$ & 1.832147000000 & -3.000288000000 & 1.130288000000 & $\mathrm{H}$ & -1.182400000000 & -1.115887000000 & -2.393250000000 \\
\hline $\mathrm{H}$ & 1.323710000000 & -1.306625000000 & 1.418971000000 & $\mathrm{H}$ & -1.009932000000 & 0.269931000000 & -1.297125000000 \\
\hline $\mathrm{H}$ & -0.338008000000 & -3.463782000000 & -0.014166000000 & $\mathrm{H}$ & -0.030817000000 & -2.556084000000 & -0.643990000000 \\
\hline $\mathrm{H}$ & -0.253782000000 & -1.845227000000 & -1.924740000000 & $\mathrm{H}$ & -0.365165000000 & -0.864045000000 & 1.234290000000 \\
\hline $\mathrm{H}$ & -0.164981000000 & -0.478966000000 & -0.772680000000 & $\mathrm{H}$ & 1.004148000000 & 0.058789000000 & 0.562690000000 \\
\hline $\mathrm{C}$ & 2.590092000000 & -0.842301000000 & -1.067616000000 & $\mathrm{C}$ & -3.400975000000 & -0.184849000000 & -0.418620000000 \\
\hline $\mathrm{H}$ & 2.470773000000 & -0.717365000000 & -2.151722000000 & $\mathrm{H}$ & -4.132856000000 & -0.695852000000 & 0.214566000000 \\
\hline $\mathrm{O}$ & -1.280649000000 & -2.163446000000 & 1.166379000000 & $\mathrm{O}$ & 1.271480000000 & -1.478758000000 & -1.688057000000 \\
\hline $\mathrm{C}$ & -1.547410000000 & -0.813683000000 & 1.543796000000 & $\mathrm{C}$ & 1.650677000000 & -0.251972000000 & -2.333100000000 \\
\hline $\mathrm{H}$ & -0.624014000000 & -0.227112000000 & 1.618426000000 & $\mathrm{H}$ & 0.779453000000 & 0.244622000000 & -2.776683000000 \\
\hline $\mathrm{H}$ & -1.965963000000 & -0.879617000000 & 2.555336000000 & $\mathrm{H}$ & 2.281006000000 & -0.590102000000 & -3.161956000000 \\
\hline $\mathrm{C}$ & 2.436089000000 & 0.548365000000 & -0.446583000000 & $\mathrm{Br}$ & 1.781019000000 & -2.074190000000 & 1.591153000000 \\
\hline $\mathrm{C}$ & 2.895258000000 & 0.852899000000 & 0.846524000000 & $\mathrm{C}$ & -2.638251000000 & -2.469546000000 & -0.557830000000 \\
\hline $\mathrm{C}$ & 1.791933000000 & 1.564026000000 & -1.171571000000 & $\mathrm{~N}$ & -2.941994000000 & -3.600141000000 & -0.509545000000 \\
\hline $\mathrm{C}$ & 2.709667000000 & 2.125099000000 & 1.396085000000 & $\mathrm{C}$ & -2.897039000000 & 1.027145000000 & 0.360396000000 \\
\hline $\mathrm{C}$ & 1.605734000000 & 2.839080000000 & -0.628690000000 & $\mathrm{C}$ & -2.771776000000 & 2.299194000000 & -0.212292000000 \\
\hline $\mathrm{C}$ & 2.063134000000 & 3.123719000000 & 0.660946000000 & $\mathrm{C}$ & -2.553871000000 & 0.863051000000 & 1.712839000000 \\
\hline $\mathrm{H}$ & 3.416350000000 & 0.100447000000 & 1.430589000000 & $\mathrm{C}$ & -2.305498000000 & 3.380533000000 & 0.545466000000 \\
\hline $\mathrm{H}$ & 1.439332000000 & 1.356100000000 & -2.179201000000 & $\mathrm{C}$ & -2.086014000000 & 1.937054000000 & 2.469875000000 \\
\hline $\mathrm{H}$ & 3.076960000000 & 2.337504000000 & 2.396392000000 & $\mathrm{C}$ & -1.958333000000 & 3.202674000000 & 1.885542000000 \\
\hline $\mathrm{H}$ & 1.108837000000 & 3.607832000000 & -1.213928000000 & $\mathrm{H}$ & -3.042065000000 & 2.462755000000 & -1.250221000000 \\
\hline $\mathrm{H}$ & 1.924264000000 & 4.113575000000 & 1.086146000000 & $\mathrm{H}$ & -2.659410000000 & -0.115113000000 & 2.175113000000 \\
\hline $\mathrm{C}$ & -2.540823000000 & -0.108795000000 & 0.634122000000 & $\mathrm{H}$ & -2.216438000000 & 4.359830000000 & 0.084270000000 \\
\hline $\mathrm{C}$ & -2.553102000000 & 1.291946000000 & 0.569816000000 & $\mathrm{H}$ & -1.827551000000 & 1.789929000000 & 3.514245000000 \\
\hline $\mathrm{C}$ & -3.489653000000 & -0.828398000000 & -0.103604000000 & $\mathrm{H}$ & -1.597945000000 & 4.041604000000 & 2.473262000000 \\
\hline $\mathrm{C}$ & -3.497720000000 & 1.962197000000 & -0.212474000000 & $\mathrm{C}$ & 2.424222000000 & 0.734578000000 & -1.476551000000 \\
\hline $\mathrm{C}$ & -4.431739000000 & -0.160096000000 & -0.892071000000 & $\mathrm{C}$ & 2.077141000000 & 2.092164000000 & -1.473492000000 \\
\hline $\mathrm{C}$ & -4.441052000000 & 1.237080000000 & -0.947785000000 & $\mathrm{C}$ & 3.527747000000 & 0.316215000000 & -0.717672000000 \\
\hline $\mathrm{H}$ & -1.815644000000 & 1.862977000000 & 1.129370000000 & $\mathrm{C}$ & 2.815066000000 & 3.017618000000 & -0.727082000000 \\
\hline $\mathrm{H}$ & -3.481908000000 & -1.912928000000 & -0.061864000000 & $\mathrm{C}$ & 4.260013000000 & 1.235237000000 & 0.035680000000 \\
\hline $\mathrm{H}$ & -3.491444000000 & 3.047855000000 & -0.253786000000 & $\mathrm{C}$ & 3.906175000000 & 2.589812000000 & 0.033265000000 \\
\hline $\mathrm{H}$ & -5.159435000000 & -0.731751000000 & -1.461517000000 & $\mathrm{H}$ & 1.222940000000 & 2.430434000000 & -2.055367000000 \\
\hline $\mathrm{H}$ & -5.172975000000 & 1.755649000000 & -1.560325000000 & $\mathrm{H}$ & 3.801566000000 & -0.734221000000 & -0.708550000000 \\
\hline $\mathrm{C}$ & 3.967894000000 & -1.469637000000 & -0.819826000000 & $\mathrm{H}$ & 2.532539000000 & 4.066441000000 & -0.735070000000 \\
\hline $\mathrm{H}$ & 4.053768000000 & -2.403641000000 & -1.382297000000 & $\mathrm{H}$ & 5.106514000000 & 0.894994000000 & 0.625006000000 \\
\hline $\mathrm{H}$ & 4.765710000000 & -0.791098000000 & -1.137202000000 & $\mathrm{H}$ & 4.477406000000 & 3.303861000000 & 0.619344000000 \\
\hline \multirow[t]{4}{*}{$\mathrm{H}$} & 4.125437000000 & -1.701131000000 & 0.238864000000 & $\mathrm{C}$ & -4.064437000000 & 0.117825000000 & -1.765192000000 \\
\hline & & & & $\mathrm{H}$ & -4.439275000000 & -0.808684000000 & -2.209241000000 \\
\hline & & & & $\mathrm{H}$ & -4.912012000000 & 0.795476000000 & -1.626818000000 \\
\hline & & & & $\mathrm{H}$ & -3.371082000000 & 0.578709000000 & -2.474975000000 \\
\hline & & & & & & 42) & \\
\hline
\end{tabular}




\begin{tabular}{|lrr}
$\mathrm{C}$ & -0.135629000000 & -0.077680000000 \\
$\mathrm{C}$ & -0.116702000000 & 1.405963000000 \\
$\mathrm{C}$ & 0.951457000000 & 1.636907000000 \\
$\mathrm{~N}$ & 1.142179000000 & -0.503529000000 \\
$\mathrm{H}$ & 1.923131000000 & 1.313249000000 \\
$\mathrm{H}$ & 0.736530000000 & 1.137263000000 \\
$\mathrm{H}$ & 0.121242000000 & 2.022213000000 \\
$\mathrm{H}$ & -0.971500000000 & -0.230015000000 \\
$\mathrm{H}$ & -0.290013000000 & -0.723179000000 \\
$\mathrm{C}$ & 1.532546000000 & -1.942521000000 \\
$\mathrm{H}$ & 0.679933000000 & -2.553664000000 \\
$\mathrm{O}$ & -1.412857000000 & 1.853962000000 \\
$\mathrm{C}$ & -2.058453000000 & 1.214355000000 \\
$\mathrm{H}$ & -1.333849000000 & 0.760505000000 \\
$\mathrm{H}$ & -2.550124000000 & 2.026512000000 \\
$\mathrm{Br}$ & 1.167123000000 & 3.539269000000 \\
$\mathrm{C}$ & 1.504358000000 & 0.136957000000 \\
$\mathrm{~N}$ & 1.836785000000 & 0.746791000000 \\
$\mathrm{C}$ & 1.788274000000 & -2.230910000000 \\
$\mathrm{C}$ & 2.761839000000 & -1.520844000000 \\
$\mathrm{C}$ & 1.081299000000 & -3.252259000000 \\
$\mathrm{C}$ & 3.014475000000 & -1.820563000000 \\
$\mathrm{C}$ & 1.337356000000 & -3.560566000000 \\
$\mathrm{C}$ & 2.302440000000 & -2.842703000000 \\
$\mathrm{H}$ & 3.320391000000 & -0.726946000000 \\
$\mathrm{H}$ & 0.324589000000 & -3.810299000000 \\
$\mathrm{H}$ & 3.766173000000 & -1.258041000000 \\
$\mathrm{H}$ & 0.780114000000 & -4.355812000000 \\
$\mathrm{H}$ & 2.499948000000 & -3.075721000000 \\
$\mathrm{C}$ & -3.087508000000 & 0.181735000000 \\
$\mathrm{C}$ & -3.227218000000 & -1.013199000000 \\
$\mathrm{C}$ & -3.945597000000 & 0.426923000000 \\
$\mathrm{C}$ & -4.208376000000 & -1.946991000000 \\
$\mathrm{C}$ & -4.920497000000 & -0.507164000000 \\
$\mathrm{C}$ & -5.056188000000 & -1.697029000000 \\
$\mathrm{H}$ & -2.567290000000 & -1.216944000000 \\
$\mathrm{H}$ & -3.840740000000 & 1.347925000000 \\
$\mathrm{H}$ & -4.303780000000 & -2.868746000000 \\
$\mathrm{H}$ & -5.576836000000 & -0.305655000000 \\
$\mathrm{C}$ & -5.816084000000 & -2.421719000000 \\
\hline & 2.542580000000 & -2.300318000000 \\
\hline
\end{tabular}

1.060851000000 0.628261000000 $-0.434033000000$ 1.675011000000 $-0.070433000000$ $-1.377095000000$ 1.501036000000 1.753322000000 0.194267000000 1.498315000000 1.824453000000 0.244918000000 $-0.867118000000$ $-1.552177000000$ $-1.414668000000$ $-0.881266000000$ 2.786224000000 3.729694000000 0.024531000000 $-0.694691000000$ $-0.622401000000$ $-2.034547000000$ $-1.963128000000$ $-2.673114000000$ $-0.207752000000$ $-0.076407000000$ $-2.580424000000$ $-2.449588000000$ $-3.715215000000$ $-0.446815000000$ $-1.164574000000$ 0.635514000000 $-0.813851000000$ 0.992959000000 0.268364000000 $-2.004685000000$ 1.201049000000 $-1.380752000000$ 1.834840000000 0.546026000000 2.367357000000 3.430856000000 2.195136000000 2.111616000000

\section{C $\quad 0.470852000000$}

C $\quad 0.769828000000$

C -0.326793000000

$\mathrm{N}-0.270910000000$

$\mathrm{H} \quad-0.117012000000$

H -1.313870000000

H $\quad 1.767286000000$

$\mathrm{H} \quad 1.330942000000$

H $\quad-0.278506000000$

C -1.640517000000

H -1.269534000000

O $\quad 0.711662000000$

C -0.302387000000

$\mathrm{H}-1.237358000000$

H $\quad-0.504219000000$

$\mathrm{Br} \quad 3.824099000000$

C $\quad 0.589573000000$

$\mathrm{N} \quad 1.273874000000$

C -2.536315000000

C -3.466014000000

C -2.483174000000

C -4.303622000000

C -3.320840000000

C -4.230980000000

$\mathrm{H} \quad-3.561971000000$

$\mathrm{H}-1.787741000000$

H $\quad-5.018666000000$

H -3.265635000000

H $\quad-4.886356000000$

C $\quad 0.114715000000$

C -0.759446000000

C 1.329693000000

C -0.427174000000

C 1.658449000000

C $\quad 0.787433000000$

$\mathrm{H}-1.704516000000$

$\mathrm{H} \quad 2.032303000000$

$\mathrm{H} \quad-1.111810000000$

H 2.602714000000

H 1.048718000000

C -2.256351000000

H -1.587403000000

$\mathrm{H} \quad-3.167510000000$

H $\quad-2.528947000000$
0.717394000000 0.709152000000 1.784757000000 2.093939000000 2.661909000000 1.374906000000 1.118324000000 0.789360000000 0.003757000000 2.356912000000 2.500454000000 $-0.464984000000$ $-1.436109000000$ $-0.952587000000$ $-1.913146000000$ 0.667396000000

3.155449000000 4.062342000000 1.143572000000 0.917655000000 0.240749000000

$-0.198850000000$ $-0.874792000000$ $-1.097735000000$ 1.615958000000 0.414804000000 $-0.359907000000$

$-1.562086000000$

$-1.962739000000$

$-2.487346000000$

$-3.558326000000$

$-2.427250000000$

$-4.550372000000$

$-3.419181000000$

$-4.483652000000$

$-3.617973000000$

$-1.618838000000$

$-5.375373000000$

$-3.353622000000$

$-5.254914000000$

3.647342000000

4.499648000000

3.843434000000

3.579463000000
$-0.043567000000$ 1.470950000000 1.671167000000 0.141256000000 2.281196000000 1.880920000000 1.644216000000 $-0.714966000000$ $-0.377332000000$ $-0.656817000000$ $-1.674080000000$ 2.223560000000 1.947788000000 1.633647000000 2.911833000000 $-0.555387000000$ $-0.123276000000$ $-0.353413000000$ $-0.628912000000$ 0.400715000000 $-1.704949000000$ 0.363392000000 $-1.741613000000$ $-0.705026000000$ 1.225815000000 $-2.521569000000$ 1.164042000000 $-2.579553000000$ $-0.732842000000$ 0.931276000000 0.685512000000 0.239271000000 $-0.237949000000$ $-0.693513000000$ $-0.931634000000$ 1.221438000000 0.419804000000 $-0.413719000000$ $-1.225877000000$ $-1.650364000000$ $-0.129367000000$ $-0.265012000000$ $-0.699663000000$ 0.926534000000 


\begin{tabular}{crrr}
$\mathrm{C}$ & 0.320539000000 & -0.516631000000 & 1.863466000000 \\
$\mathrm{C}$ & -0.048425000000 & -1.931892000000 & 1.342107000000 \\
$\mathrm{C}$ & -1.410965000000 & -1.363762000000 & 0.886213000000 \\
$\mathrm{~N}$ & -1.206699000000 & -0.175909000000 & 1.889414000000 \\
$\mathrm{H}$ & -2.323432000000 & -1.922787000000 & 1.083029000000 \\
$\mathrm{H}$ & -1.433589000000 & -0.946375000000 & -0.126443000000 \\
$\mathrm{H}$ & -0.182233000000 & -2.616406000000 & 2.183479000000 \\
$\mathrm{H}$ & 0.791626000000 & -0.397881000000 & 2.837961000000 \\
$\mathrm{H}$ & 0.771688000000 & 0.136882000000 & 1.118668000000 \\
$\mathrm{C}$ & -1.743518000000 & 1.302957000000 & 1.475174000000 \\
$\mathrm{H}$ & -1.724110000000 & 1.807599000000 & 2.443612000000 \\
$\mathrm{O}$ & 0.757311000000 & -2.623465000000 & 0.443961000000 \\
$\mathrm{C}$ & 1.238813000000 & -1.959949000000 & -0.740781000000 \\
$\mathrm{H}$ & 0.481089000000 & -1.288281000000 & -1.157361000000 \\
$\mathrm{H}$ & 1.367930000000 & -2.772660000000 & -1.462198000000 \\
$\mathrm{Br}$ & -2.620142000000 & -1.050560000000 & -2.328361000000 \\
$\mathrm{C}$ & -1.749512000000 & -0.480018000000 & 3.130925000000 \\
$\mathrm{~N}$ & -2.202933000000 & -0.709536000000 & 4.173719000000 \\
$\mathrm{C}$ & -0.792498000000 & 2.009738000000 & 0.547446000000 \\
$\mathrm{C}$ & -0.865380000000 & 1.879612000000 & -0.849404000000 \\
$\mathrm{C}$ & 0.135044000000 & 2.907529000000 & 1.106626000000 \\
$\mathrm{C}$ & -0.018996000000 & 2.635883000000 & -1.663518000000 \\
$\mathrm{C}$ & 0.974710000000 & 3.664088000000 & 0.288953000000 \\
$\mathrm{C}$ & 0.897657000000 & 3.528074000000 & -1.100598000000 \\
$\mathrm{H}$ & -1.567271000000 & 1.192382000000 & -1.316091000000 \\
$\mathrm{H}$ & 0.186169000000 & 3.028112000000 & 2.185833000000 \\
$\mathrm{H}$ & -0.084342000000 & 2.526860000000 & -2.741766000000 \\
$\mathrm{H}$ & 1.679314000000 & 4.359717000000 & 0.733975000000 \\
$\mathrm{H}$ & 1.544156000000 & 4.120523000000 & -1.741123000000 \\
$\mathrm{C}$ & 2.556438000000 & -1.242182000000 & -0.520266000000 \\
$\mathrm{C}$ & 2.860676000000 & -0.093301000000 & -1.263188000000 \\
$\mathrm{C}$ & 3.510000000000 & -1.743888000000 & 0.377858000000 \\
$\mathrm{C}$ & 4.097697000000 & 0.541669000000 & -1.115903000000 \\
$\mathrm{C}$ & 4.743038000000 & -1.104442000000 & 0.532326000000 \\
$\mathrm{C}$ & 5.041917000000 & 0.039056000000 & -0.216038000000 \\
$\mathrm{H}$ & 2.127356000000 & 0.308875000000 & -1.957066000000 \\
$\mathrm{H}$ & 3.283389000000 & -2.635570000000 & 0.954292000000 \\
$\mathrm{H}$ & 4.319295000000 & 1.430933000000 & -1.698921000000 \\
\hline & -3.472572000000 & -1.503021000000 & 1.231664000000 \\
\hline
\end{tabular}


Table S6. Optimized stationary point geometries shown in Scheme 12. PCM/B3LYP/6-31++G(d,p) calculations (Chloroform, $25^{\circ} \mathrm{C}$ ). Coordinates in angstroms. Sum of electronic and thermal Free Energies are in parentheses.

\begin{tabular}{|c|c|c|c|c|c|c|c|}
\hline \multicolumn{4}{|c|}{$25(-788.96024)$} & \multicolumn{4}{|c|}{$11(-2664.55987)$} \\
\hline $\mathrm{C}$ & -2.223777000000 & 0.669307000000 & -0.156180000000 & $\mathrm{Br}$ & 0.000000000000 & 0.651724000000 & 0.000000000000 \\
\hline $\mathrm{C}$ & -3.134308000000 & -0.557349000000 & -0.430508000000 & $\mathrm{C}$ & 0.008648000000 & -1.127893000000 & 0.000000000000 \\
\hline $\mathrm{C}$ & -1.885421000000 & -1.418477000000 & -0.184315000000 & $\mathrm{~N}$ & -0.007413000000 & -2.291856000000 & 0.000000000000 \\
\hline $\mathrm{N}$ & -1.050150000000 & -0.208390000000 & -0.408402000000 & & & & \\
\hline $\mathrm{H}$ & -1.682721000000 & -2.256389000000 & -0.857203000000 & & & & \\
\hline $\mathrm{H}$ & -1.866516000000 & -1.769052000000 & 0.859660000000 & & & & \\
\hline $\mathrm{H}$ & -3.488007000000 & -0.594811000000 & -1.469900000000 & & & & \\
\hline $\mathrm{H}$ & -2.293887000000 & 1.544302000000 & -0.808974000000 & & & & \\
\hline $\mathrm{H}$ & -2.308199000000 & 0.983665000000 & 0.896790000000 & & & & \\
\hline $\mathrm{C}$ & 0.129991000000 & -0.030621000000 & 0.435735000000 & & & & \\
\hline $\mathrm{H}$ & -0.156239000000 & -0.080364000000 & 1.503665000000 & & & & \\
\hline $\mathrm{C}$ & 0.758546000000 & 1.344900000000 & 0.205966000000 & & & & \\
\hline $\mathrm{C}$ & 0.821951000000 & 1.917976000000 & -1.072597000000 & & & & \\
\hline $\mathrm{C}$ & 1.311004000000 & 2.048346000000 & 1.285154000000 & & & & \\
\hline $\mathrm{C}$ & 1.431005000000 & 3.160567000000 & -1.266812000000 & & & & \\
\hline $\mathrm{C}$ & 1.924421000000 & 3.290139000000 & 1.094089000000 & & & & \\
\hline $\mathrm{C}$ & 1.987397000000 & 3.850755000000 & -0.184803000000 & & & & \\
\hline $\mathrm{H}$ & 0.382306000000 & 1.390395000000 & -1.912928000000 & & & & \\
\hline $\mathrm{H}$ & 1.260539000000 & 1.622745000000 & 2.284484000000 & & & & \\
\hline $\mathrm{H}$ & 1.468661000000 & 3.591098000000 & -2.263746000000 & & & & \\
\hline $\mathrm{H}$ & 2.346053000000 & 3.819898000000 & 1.943716000000 & & & & \\
\hline $\mathrm{H}$ & 2.459884000000 & 4.816997000000 & -0.336348000000 & & & & \\
\hline $\mathrm{O}$ & -4.195847000000 & -0.820636000000 & 0.458991000000 & & & & \\
\hline $\mathrm{C}$ & -5.328139000000 & 0.015836000000 & 0.242366000000 & & & & \\
\hline $\mathrm{H}$ & -6.094943000000 & -0.293250000000 & 0.955225000000 & & & & \\
\hline $\mathrm{H}$ & -5.084641000000 & 1.073558000000 & 0.412967000000 & & & & \\
\hline $\mathrm{H}$ & -5.715943000000 & -0.100202000000 & -0.779795000000 & & & & \\
\hline $\mathrm{C}$ & 1.134657000000 & -1.158255000000 & 0.192710000000 & & & & \\
\hline $\mathrm{C}$ & 1.737850000000 & -1.807430000000 & 1.277760000000 & & & & \\
\hline $\mathrm{C}$ & 1.485479000000 & -1.553344000000 & -1.107050000000 & & & & \\
\hline $\mathrm{C}$ & 2.678620000000 & -2.822736000000 & 1.074429000000 & & & & \\
\hline $\mathrm{C}$ & 2.420892000000 & -2.569406000000 & -1.314163000000 & & & & \\
\hline $\mathrm{C}$ & 3.023639000000 & -3.206925000000 & -0.223716000000 & & & & \\
\hline $\mathrm{H}$ & 1.468735000000 & -1.519742000000 & 2.291176000000 & & & & \\
\hline $\mathrm{H}$ & 1.015749000000 & -1.067445000000 & -1.956246000000 & & & & \\
\hline $\mathrm{H}$ & 3.134306000000 & -3.314862000000 & 1.929063000000 & & & & \\
\hline $\mathrm{H}$ & 2.678943000000 & -2.865147000000 & -2.327332000000 & & & & \\
\hline $\mathrm{H}$ & 3.750907000000 & -3.997279000000 & -0.385782000000 & & & & \\
\hline \multicolumn{4}{|c|}{ 28a $(-3453.49504)$} & \multicolumn{4}{|c|}{ TS14 (-3453.46659) } \\
\hline
\end{tabular}




\begin{tabular}{|lrrr}
$\mathrm{C}$ & 1.867169000000 & -1.044323000000 & -1.838220000000 \\
$\mathrm{C}$ & 2.513636000000 & 0.354135000000 & -1.661249000000 \\
$\mathrm{C}$ & 1.297903000000 & 0.774176000000 & -0.829764000000 \\
$\mathrm{~N}$ & 0.492683000000 & -0.455747000000 & -1.394698000000 \\
$\mathrm{H}$ & 0.784409000000 & 1.720021000000 & -0.988866000000 \\
$\mathrm{H}$ & 1.451946000000 & 0.601068000000 & 0.232766000000 \\
$\mathrm{H}$ & 2.596327000000 & 0.898398000000 & -2.609524000000 \\
$\mathrm{H}$ & 1.808542000000 & -1.521056000000 & -2.815679000000 \\
$\mathrm{H}$ & 2.183475000000 & -1.738041000000 & -1.058601000000 \\
$\mathrm{C}$ & -0.409026000000 & -1.416181000000 & -0.432960000000 \\
$\mathrm{H}$ & -0.311730000000 & -2.358656000000 & -0.974693000000 \\
$\mathrm{C}$ & -1.860759000000 & -0.994658000000 & -0.481568000000 \\
$\mathrm{C}$ & -2.311177000000 & 0.291078000000 & -0.143758000000 \\
$\mathrm{C}$ & -2.793563000000 & -1.970761000000 & -0.869445000000 \\
$\mathrm{C}$ & -3.675064000000 & 0.585022000000 & -0.195108000000 \\
$\mathrm{C}$ & -4.157299000000 & -1.675783000000 & -0.902540000000 \\
$\mathrm{C}$ & -4.600891000000 & -0.394145000000 & -0.565464000000 \\
$\mathrm{H}$ & -1.626607000000 & 1.085462000000 & 0.147663000000 \\
$\mathrm{H}$ & -2.453862000000 & -2.966213000000 & -1.143438000000 \\
$\mathrm{H}$ & -4.008439000000 & 1.586199000000 & 0.059281000000 \\
$\mathrm{H}$ & -4.866797000000 & -2.441614000000 & -1.200596000000 \\
$\mathrm{H}$ & -5.660300000000 & -0.158289000000 & -0.597794000000 \\
$\mathrm{O}$ & 3.698554000000 & 0.411036000000 & -0.929581000000 \\
$\mathrm{C}$ & 4.881698000000 & 0.237955000000 & -1.718739000000 \\
$\mathrm{H}$ & 5.725159000000 & 0.360302000000 & -1.039116000000 \\
$\mathrm{H}$ & 4.919179000000 & -0.762638000000 & -2.167593000000 \\
$\mathrm{H}$ & 4.935254000000 & 0.996877000000 & -2.508965000000 \\
$\mathrm{Br}$ & -0.298337000000 & 3.129289000000 & 1.124133000000 \\
$\mathrm{C}$ & -0.243652000000 & -0.087071000000 & -2.515281000000 \\
$\mathrm{~N}$ & -0.799449000000 & 0.205486000000 & -3.490204000000 \\
$\mathrm{C}$ & 0.237684000000 & -1.574634000000 & 0.921166000000 \\
$\mathrm{C}$ & 0.143860000000 & -0.600790000000 & 1.931651000000 \\
$\mathrm{C}$ & 0.913736000000 & -2.777144000000 & 1.193074000000 \\
$\mathrm{C}$ & 0.729267000000 & -0.826840000000 & 3.177322000000 \\
$\mathrm{C}$ & 1.482387000000 & -3.007027000000 & 2.447585000000 \\
$\mathrm{C}$ & 1.389082000000 & -2.030997000000 & 3.442657000000 \\
$\mathrm{H}$ & -0.361527000000 & 0.344408000000 & 1.759087000000 \\
$\mathrm{H}$ & 0.977451000000 & -3.547723000000 & 0.428867000000 \\
& 0.659764000000 & -0.059844000000 & 3.941981000000 \\
$\mathrm{H}$ & 1.989676000000 & -3.946070000000 & 2.645566000000 \\
& 1.828854000000 & -2.206224000000 & 4.419797000000 \\
& & & \\
\hline
\end{tabular}

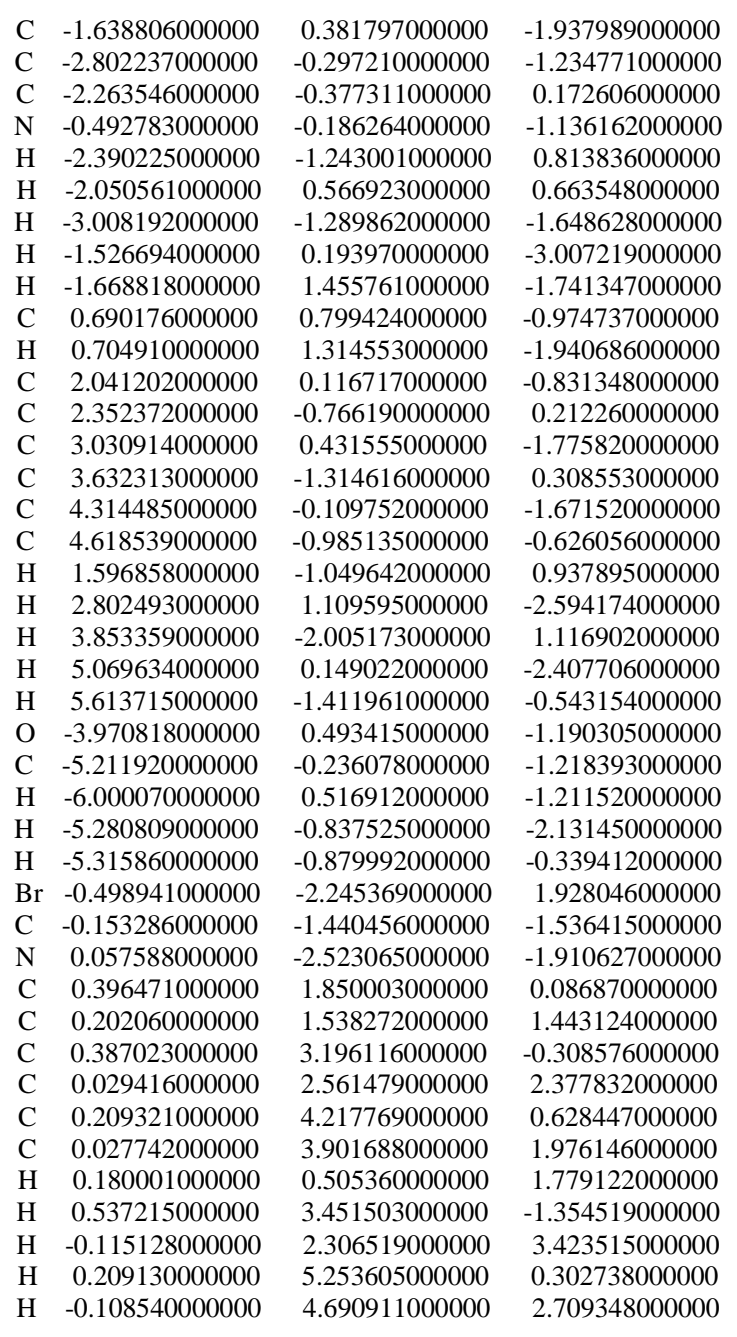

C -1.638806000000

C -2.802237000000

C -2.263546000000

$\mathrm{H}-2.050561000000$

H -3.008192000000

$-1.526694000000$

C 4.618539000000

$\mathrm{H} \quad 1.596858000000$

H 2.802493000000

H 3.853359000000

H 5.069634000000

H 5.613715000000

(1)

H $\quad-5.315860000000$

$\mathrm{Br} \quad-0.498941000000$

C -0.153286000000

N 0.057588000000

C 0396471000000

C $\quad 0.202060000000$

C $\quad 0.387023000000$

C 0.029416000000

C $\quad 0.209321000000$

C 0.027742000000

H 0.180001000000

$\mathrm{H} \quad 0.209130000000$

H -0.108540000000
$-1.937989000000$

$-1.234771000000$

0.663548000000

$-1.648628000000$

$-0.974737000000$

940686000000 0.831348000000

0.308553000000

1.671520000000

0.626056000000

1.116902000000

.407706000000

0.543154000000

$-1.211520000000$

2.131450000000

0.339412000000

$-1.910627000000$

86870000000

$-0.308576000000$

2.377832000000

0.628447000000

$-1.354519000000$

515000000

2.709348000000 


\begin{tabular}{|c|c|c|c|c|c|c|c|}
\hline $\mathrm{C}$ & 1.824583000000 & -1.830718000000 & -1.296440000000 & $\mathrm{C}$ & -1.322106000000 & 0.386658000000 & -1.535525000000 \\
\hline $\mathrm{C}$ & 2.518671000000 & -0.516600000000 & -1.750290000000 & $\mathrm{C}$ & -2.620897000000 & -0.077799000000 & -0.859921000000 \\
\hline $\mathrm{C}$ & 1.322668000000 & 0.258945000000 & -1.184489000000 & $\mathrm{C}$ & -2.667357000000 & 0.181599000000 & 0.651696000000 \\
\hline $\mathrm{N}$ & 0.511370000000 & -1.052466000000 & -1.108043000000 & $\mathrm{~N}$ & -0.129092000000 & -0.365431000000 & -1.083335000000 \\
\hline $\mathrm{H}$ & 0.828038000000 & 1.043032000000 & -1.754628000000 & $\mathrm{H}$ & -3.695560000000 & 0.242056000000 & 1.002176000000 \\
\hline $\mathrm{H}$ & 1.502507000000 & 0.602641000000 & -0.165721000000 & $\mathrm{H}$ & -2.134361000000 & 1.087373000000 & 0.938203000000 \\
\hline $\mathrm{H}$ & 2.594761000000 & -0.446509000000 & -2.842681000000 & $\mathrm{H}$ & -2.785549000000 & -1.145755000000 & -1.058494000000 \\
\hline $\mathrm{H}$ & 1.723597000000 & -2.668967000000 & -1.985035000000 & $\mathrm{H}$ & -1.446297000000 & 0.301191000000 & -2.621635000000 \\
\hline $\mathrm{H}$ & 2.174170000000 & -2.155707000000 & -0.315159000000 & $\mathrm{H}$ & -1.155298000000 & 1.437794000000 & -1.295539000000 \\
\hline $\mathrm{C}$ & -0.455479000000 & -1.444117000000 & 0.578029000000 & $\mathrm{C}$ & 1.186202000000 & 0.354505000000 & -1.077762000000 \\
\hline $\mathrm{H}$ & -0.223052000000 & -2.505718000000 & 0.516572000000 & $\mathrm{H}$ & 1.425840000000 & 0.634804000000 & -2.112050000000 \\
\hline $\mathrm{C}$ & -1.878830000000 & -1.187277000000 & 0.292759000000 & $\mathrm{C}$ & 2.310452000000 & -0.543665000000 & -0.572270000000 \\
\hline $\mathrm{C}$ & -2.433535000000 & 0.083909000000 & 0.040758000000 & $\mathrm{C}$ & 2.160001000000 & -1.370829000000 & 0.550846000000 \\
\hline $\mathrm{C}$ & -2.727390000000 & -2.314682000000 & 0.307905000000 & $\mathrm{C}$ & 3.550415000000 & -0.503639000000 & -1.223496000000 \\
\hline $\mathrm{C}$ & -3.806835000000 & 0.214481000000 & -0.152406000000 & $\mathrm{C}$ & 3.235753000000 & -2.127400000000 & 1.019451000000 \\
\hline $\mathrm{C}$ & -4.103802000000 & -2.170786000000 & 0.142216000000 & $\mathrm{C}$ & 4.628155000000 & -1.259316000000 & -0.753971000000 \\
\hline $\mathrm{C}$ & -4.646282000000 & -0.903142000000 & -0.085702000000 & $\mathrm{C}$ & 4.472814000000 & -2.075200000000 & 0.369686000000 \\
\hline $\mathrm{H}$ & -1.810965000000 & 0.969926000000 & -0.063671000000 & $\mathrm{H}$ & 1.202982000000 & -1.432873000000 & 1.057899000000 \\
\hline $\mathrm{H}$ & -2.307736000000 & -3.302806000000 & 0.475065000000 & $\mathrm{H}$ & 3.676436000000 & 0.124974000000 & -2.101323000000 \\
\hline $\mathrm{H}$ & -4.219714000000 & 1.196214000000 & -0.361522000000 & $\mathrm{H}$ & 3.103490000000 & -2.766126000000 & 1.887823000000 \\
\hline $\mathrm{H}$ & -4.745945000000 & -3.045240000000 & 0.175310000000 & $\mathrm{H}$ & 5.582055000000 & -1.216660000000 & -1.271368000000 \\
\hline $\mathrm{H}$ & -5.716657000000 & -0.787464000000 & -0.226786000000 & $\mathrm{H}$ & 5.306793000000 & -2.667602000000 & 0.734301000000 \\
\hline $\mathrm{O}$ & 3.724261000000 & -0.181781000000 & -1.130437000000 & $\mathrm{O}$ & -3.643736000000 & 0.692377000000 & -1.488353000000 \\
\hline $\mathrm{C}$ & 4.868252000000 & -0.811469000000 & -1.714657000000 & $\mathrm{C}$ & -4.926358000000 & 0.065647000000 & -1.514533000000 \\
\hline $\mathrm{H}$ & 5.740262000000 & -0.422255000000 & -1.188310000000 & $\mathrm{H}$ & -5.581626000000 & 0.722421000000 & -2.088998000000 \\
\hline $\mathrm{H}$ & 4.829403000000 & -1.901820000000 & -1.592752000000 & $\mathrm{H}$ & -4.871317000000 & -0.915023000000 & -2.004501000000 \\
\hline $\mathrm{H}$ & 4.946318000000 & -0.567360000000 & -2.781705000000 & $\mathrm{H}$ & -5.342397000000 & -0.060019000000 & -0.506671000000 \\
\hline $\mathrm{Br}$ & -0.519300000000 & 3.206020000000 & -0.557058000000 & $\mathrm{Br}$ & -1.854966000000 & -1.291750000000 & 1.693970000000 \\
\hline $\mathrm{C}$ & -0.363493000000 & -1.236706000000 & -2.141423000000 & $\mathrm{C}$ & -0.122853000000 & -1.651199000000 & -1.452312000000 \\
\hline $\mathrm{N}$ & -1.112133000000 & -1.437395000000 & -3.008425000000 & $\mathrm{~N}$ & -0.188976000000 & -2.780409000000 & -1.752123000000 \\
\hline $\mathrm{C}$ & 0.329716000000 & -0.778691000000 & 1.623062000000 & $\mathrm{C}$ & 1.092401000000 & 1.646719000000 & -0.267847000000 \\
\hline $\mathrm{C}$ & 0.126136000000 & 0.557368000000 & 2.032282000000 & $\mathrm{C}$ & 0.705726000000 & 1.652898000000 & 1.081345000000 \\
\hline $\mathrm{C}$ & 1.318955000000 & -1.547227000000 & 2.280057000000 & $\mathrm{C}$ & 1.440862000000 & 2.858876000000 & -0.877057000000 \\
\hline $\mathrm{C}$ & 0.898749000000 & 1.101874000000 & 3.054191000000 & $\mathrm{C}$ & 0.688302000000 & 2.846597000000 & 1.804595000000 \\
\hline $\mathrm{C}$ & 2.075456000000 & -1.002412000000 & 3.313957000000 & $\mathrm{C}$ & 1.425681000000 & 4.055667000000 & -0.152821000000 \\
\hline $\mathrm{C}$ & 1.865563000000 & 0.325265000000 & 3.702038000000 & $\mathrm{C}$ & 1.049737000000 & 4.052023000000 & 1.191790000000 \\
\hline $\mathrm{H}$ & -0.611795000000 & 1.183031000000 & 1.548122000000 & $\mathrm{H}$ & 0.429157000000 & 0.725801000000 & 1.571899000000 \\
\hline $\mathrm{H}$ & 1.468601000000 & -2.585724000000 & 1.997918000000 & $\mathrm{H}$ & 1.732661000000 & 2.869440000000 & -1.924212000000 \\
\hline $\mathrm{H}$ & 0.742203000000 & 2.135491000000 & 3.343907000000 & $\mathrm{H}$ & 0.392157000000 & 2.835521000000 & 2.849635000000 \\
\hline $\mathrm{H}$ & 2.819813000000 & -1.609968000000 & 3.818361000000 & $\mathrm{H}$ & 1.703071000000 & 4.985018000000 & -0.641440000000 \\
\hline $\mathrm{H}$ & 2.454335000000 & 0.753695000000 & 4.507437000000 & $\mathrm{H}$ & 1.036362000000 & 4.977942000000 & 1.758997000000 \\
\hline \multicolumn{4}{|c|}{$22(-379.93402)$} & \multicolumn{4}{|c|}{$27(-3073.62745)$} \\
\hline $\mathrm{C}$ & 0.069712000000 & -0.958002000000 & 0.216788000000 & $\mathrm{C}$ & 0.020613000000 & 0.405300000000 & 0.605213000000 \\
\hline $\mathrm{C}$ & -0.870725000000 & 0.256326000000 & 0.490382000000 & $\mathrm{H}$ & 0.072413000000 & 0.690002000000 & 1.653871000000 \\
\hline $\mathrm{C}$ & 0.300048000000 & 1.196595000000 & 0.128399000000 & $\mathrm{C}$ & 1.268975000000 & -0.358754000000 & 0.241079000000 \\
\hline $\mathrm{N}$ & 1.102960000000 & 0.002817000000 & -0.265605000000 & $\mathrm{C}$ & 1.301034000000 & -1.389836000000 & -0.708282000000 \\
\hline $\mathrm{H}$ & 0.725672000000 & 1.746462000000 & 0.972599000000 & $\mathrm{C}$ & 2.438029000000 & -0.081591000000 & 0.972425000000 \\
\hline $\mathrm{H}$ & 0.097927000000 & 1.870365000000 & -0.707495000000 & $\mathrm{C}$ & 2.476901000000 & -2.114226000000 & -0.928456000000 \\
\hline $\mathrm{H}$ & -1.219548000000 & 0.328498000000 & 1.527549000000 & $\mathrm{C}$ & 3.610523000000 & -0.801090000000 & 0.751999000000 \\
\hline $\mathrm{H}$ & 0.378655000000 & -1.510801000000 & 1.109065000000 & $\mathrm{C}$ & 3.637398000000 & -1.815777000000 & -0.211510000000 \\
\hline $\mathrm{H}$ & -0.265702000000 & -1.646484000000 & -0.563163000000 & $\mathrm{H}$ & 0.408032000000 & -1.653439000000 & -1.261786000000 \\
\hline $\mathrm{O}$ & -1.944118000000 & 0.415249000000 & -0.406346000000 & $\mathrm{H}$ & 2.425145000000 & 0.709861000000 & 1.717315000000 \\
\hline $\mathrm{C}$ & -3.057015000000 & -0.427427000000 & -0.111731000000 & $\mathrm{H}$ & 2.482282000000 & -2.911397000000 & -1.666167000000 \\
\hline $\mathrm{H}$ & -3.830202000000 & -0.196185000000 & -0.845877000000 & $\mathrm{H}$ & 4.501570000000 & -0.570697000000 & 1.328492000000 \\
\hline $\mathrm{H}$ & -2.791036000000 & -1.489711000000 & -0.193172000000 & $\mathrm{H}$ & 4.547668000000 & -2.382419000000 & -0.383609000000 \\
\hline $\mathrm{H}$ & -3.441428000000 & -0.229689000000 & 0.898261000000 & $\mathrm{Br}$ & 0.125111000000 & 2.247537000000 & -0.278467000000 \\
\hline $\mathrm{C}$ & 2.413448000000 & -0.134280000000 & -0.144529000000 & $\mathrm{C}$ & -1.323165000000 & -0.204310000000 & 0.316953000000 \\
\hline \multirow[t]{10}{*}{$\mathrm{N}$} & 3.577868000000 & -0.259062000000 & -0.080516000000 & $\mathrm{C}$ & -1.796179000000 & -0.434166000000 & -0.983839000000 \\
\hline & & & & $\mathrm{C}$ & -2.111308000000 & -0.6116760000000 & 1.403568000000 \\
\hline & & & & $\mathrm{C}$ & -3.025004000000 & -1.061208000000 & -1.191031000000 \\
\hline & & & & $\mathrm{C}$ & -3.335993000000 & -1.253280000000 & 1.198268000000 \\
\hline & & & & $\mathrm{C}$ & -3.791540000000 & -1.488154000000 & -0.100834000000 \\
\hline & & & & $\mathrm{H}$ & -1.222371000000 & -0.084928000000 & -1.836336000000 \\
\hline & & & & $\mathrm{H}$ & -1.760638000000 & -0.434676000000 & 2.417305000000 \\
\hline & & & & $\mathrm{H}$ & -3.380813000000 & -1.224438000000 & -2.204120000000 \\
\hline & & & & $\mathrm{H}$ & -3.927448000000 & -1.569984000000 & 2.052007000000 \\
\hline & & & & $\mathrm{H}$ & -4.746422000000 & -1.979065000000 & -0.263963000000 \\
\hline
\end{tabular}


Table S7. Optimized stationary point geometries shown in Scheme 12. PCM/B3LYP/6-31++G(d,p) calculations (Chloroform, $25^{\circ} \mathrm{C}$ ). Coordinates in angstroms. Sum of electronic and thermal Free Energies are in parentheses.

\begin{tabular}{|c|c|c|c|c|c|c|c|}
\hline \multicolumn{4}{|c|}{$\mathbf{2 8 b}(-3453.49327)$} & \multicolumn{4}{|c|}{ TS16 (-3453.49279) } \\
\hline $\mathrm{C}$ & -1.280876000000 & -0.727694000000 & 0.117437000000 & $\mathrm{C}$ & -1.468196000000 & -0.787565000000 & -0.122029000000 \\
\hline $\mathrm{C}$ & -1.484893000000 & -0.521334000000 & 1.636836000000 & $\mathrm{C}$ & -1.595456000000 & -0.666462000000 & 1.407702000000 \\
\hline $\mathrm{C}$ & 0.036598000000 & -0.489963000000 & 1.840047000000 & $\mathrm{C}$ & -0.078374000000 & -0.687033000000 & 1.643646000000 \\
\hline $\mathrm{N}$ & 0.289564000000 & -0.927542000000 & 0.364059000000 & $\mathrm{~N}$ & 0.315869000000 & -1.003814000000 & 0.194210000000 \\
\hline $\mathrm{H}$ & 0.493710000000 & -1.180018000000 & 2.546703000000 & $\mathrm{H}$ & 0.315811000000 & -1.430136000000 & 2.335654000000 \\
\hline $\mathrm{H}$ & 0.403104000000 & 0.527987000000 & 1.960434000000 & $\mathrm{H}$ & 0.280694000000 & 0.311106000000 & 1.888553000000 \\
\hline $\mathrm{H}$ & -1.973651000000 & -1.379044000000 & 2.110894000000 & $\mathrm{H}$ & -2.119613000000 & -1.529311000000 & 1.830315000000 \\
\hline $\mathrm{H}$ & -1.709684000000 & -1.597373000000 & -0.371111000000 & $\mathrm{H}$ & -1.715218000000 & -1.703391000000 & -0.638066000000 \\
\hline $\mathrm{H}$ & -1.417194000000 & 0.172044000000 & -0.474355000000 & $\mathrm{H}$ & -1.504330000000 & 0.125443000000 & -0.700200000000 \\
\hline $\mathrm{C}$ & 1.272915000000 & -0.073698000000 & -0.583607000000 & $\mathrm{C}$ & 1.305635000000 & -0.086872000000 & -0.607571000000 \\
\hline $\mathrm{H}$ & 0.948562000000 & -0.422929000000 & -1.565940000000 & $\mathrm{H}$ & 1.077043000000 & -0.372615000000 & -1.636600000000 \\
\hline $\mathrm{C}$ & 2.709708000000 & -0.502723000000 & -0.369427000000 & $\mathrm{C}$ & 2.751155000000 & -0.460652000000 & -0.325929000000 \\
\hline $\mathrm{C}$ & 3.317029000000 & -0.588421000000 & 0.893501000000 & $\mathrm{C}$ & 3.264232000000 & -0.649310000000 & 0.966379000000 \\
\hline $\mathrm{C}$ & 3.468572000000 & -0.803456000000 & -1.510548000000 & $\mathrm{C}$ & 3.613653000000 & -0.595197000000 & -1.423783000000 \\
\hline $\mathrm{C}$ & 4.658087000000 & -0.960133000000 & 1.007930000000 & $\mathrm{C}$ & 4.612976000000 & -0.960358000000 & 1.153769000000 \\
\hline $\mathrm{C}$ & 4.812785000000 & -1.162041000000 & -1.395464000000 & $\mathrm{C}$ & 4.964272000000 & -0.894687000000 & -1.235618000000 \\
\hline $\mathrm{C}$ & 5.410441000000 & -1.243018000000 & -0.135211000000 & $\mathrm{C}$ & 5.467777000000 & -1.078456000000 & 0.054824000000 \\
\hline $\mathrm{H}$ & 2.759224000000 & -0.376509000000 & 1.799853000000 & $\mathrm{H}$ & 2.624981000000 & -0.565834000000 & 1.838974000000 \\
\hline $\mathrm{H}$ & 3.007945000000 & -0.754189000000 & -2.493386000000 & $\mathrm{H}$ & 3.227905000000 & -0.464970000000 & -2.431202000000 \\
\hline $\mathrm{H}$ & 5.111982000000 & -1.028964000000 & 1.991565000000 & $\mathrm{H}$ & 4.992933000000 & -1.109238000000 & 2.159837000000 \\
\hline $\mathrm{H}$ & 5.386327000000 & -1.389023000000 & -2.288589000000 & $\mathrm{H}$ & 5.617381000000 & -0.995101000000 & -2.096935000000 \\
\hline $\mathrm{H}$ & 6.453146000000 & -1.530407000000 & -0.043266000000 & $\mathrm{H}$ & 6.515752000000 & -1.319838000000 & 0.203029000000 \\
\hline $\mathrm{O}$ & -2.053714000000 & 0.687614000000 & 2.043004000000 & $\mathrm{O}$ & -2.118178000000 & 0.541214000000 & 1.891920000000 \\
\hline $\mathrm{C}$ & -3.469564000000 & 0.631393000000 & 2.300381000000 & $\mathrm{C}$ & -3.477825000000 & 0.495634000000 & 2.356571000000 \\
\hline $\mathrm{H}$ & -3.767339000000 & 1.649882000000 & 2.552805000000 & $\mathrm{H}$ & -3.698732000000 & 1.499319000000 & 2.723262000000 \\
\hline $\mathrm{H}$ & -4.010199000000 & 0.287194000000 & 1.413286000000 & $\mathrm{H}$ & -4.154412000000 & 0.233334000000 & 1.539967000000 \\
\hline $\mathrm{H}$ & -3.673626000000 & -0.033347000000 & 3.149684000000 & $\mathrm{H}$ & -3.576799000000 & -0.223817000000 & 3.179704000000 \\
\hline $\mathrm{Br}$ & -4.236924000000 & -0.934089000000 & -1.046433000000 & $\mathrm{Br}$ & -4.136672000000 & -0.825962000000 & -0.953827000000 \\
\hline $\mathrm{C}$ & 0.624067000000 & -2.270294000000 & 0.270394000000 & $\mathrm{C}$ & 0.630081000000 & -2.323784000000 & -0.004302000000 \\
\hline $\mathrm{N}$ & 0.884763000000 & -3.397706000000 & 0.189066000000 & $\mathrm{~N}$ & 0.867384000000 & -3.445364000000 & -0.197266000000 \\
\hline $\mathrm{C}$ & 0.979023000000 & 1.406704000000 & -0.482167000000 & $\mathrm{C}$ & 0.961108000000 & 1.384318000000 & -0.453255000000 \\
\hline $\mathrm{C}$ & 1.608486000000 & 2.241289000000 & 0.457228000000 & $\mathrm{C}$ & 1.505692000000 & 2.186295000000 & 0.562585000000 \\
\hline $\mathrm{C}$ & 0.080913000000 & 1.978281000000 & -1.400645000000 & $\mathrm{C}$ & 0.105331000000 & 1.978035000000 & -1.396510000000 \\
\hline $\mathrm{C}$ & 1.312573000000 & 3.604057000000 & 0.501850000000 & $\mathrm{C}$ & 1.172033000000 & 3.538885000000 & 0.652188000000 \\
\hline $\mathrm{C}$ & -0.211573000000 & 3.342118000000 & -1.357187000000 & $\mathrm{C}$ & -0.223974000000 & 3.331726000000 & -1.310294000000 \\
\hline $\mathrm{C}$ & 0.398583000000 & 4.156442000000 & -0.400417000000 & $\mathrm{C}$ & 0.304207000000 & 4.113949000000 & -0.280424000000 \\
\hline $\mathrm{H}$ & 2.347875000000 & 1.841164000000 & 1.141838000000 & $\mathrm{H}$ & 2.209141000000 & 1.769003000000 & 1.274868000000 \\
\hline $\mathrm{H}$ & -0.384043000000 & 1.360249000000 & -2.163994000000 & $\mathrm{H}$ & -0.295613000000 & 1.385198000000 & -2.214511000000 \\
\hline $\mathrm{H}$ & 1.804986000000 & 4.235197000000 & 1.234946000000 & $\mathrm{H}$ & 1.599465000000 & 4.144374000000 & 1.445402000000 \\
\hline $\mathrm{H}$ & -0.910073000000 & 3.764221000000 & -2.072240000000 & $\mathrm{H}$ & -0.887315000000 & 3.771486000000 & -2.048128000000 \\
\hline $\mathrm{H}$ & 0.173478000000 & 5.217770000000 & -0.365457000000 & $\mathrm{H}$ & 0.051126000000 & 5.167379000000 & -0.211637000000 \\
\hline
\end{tabular}




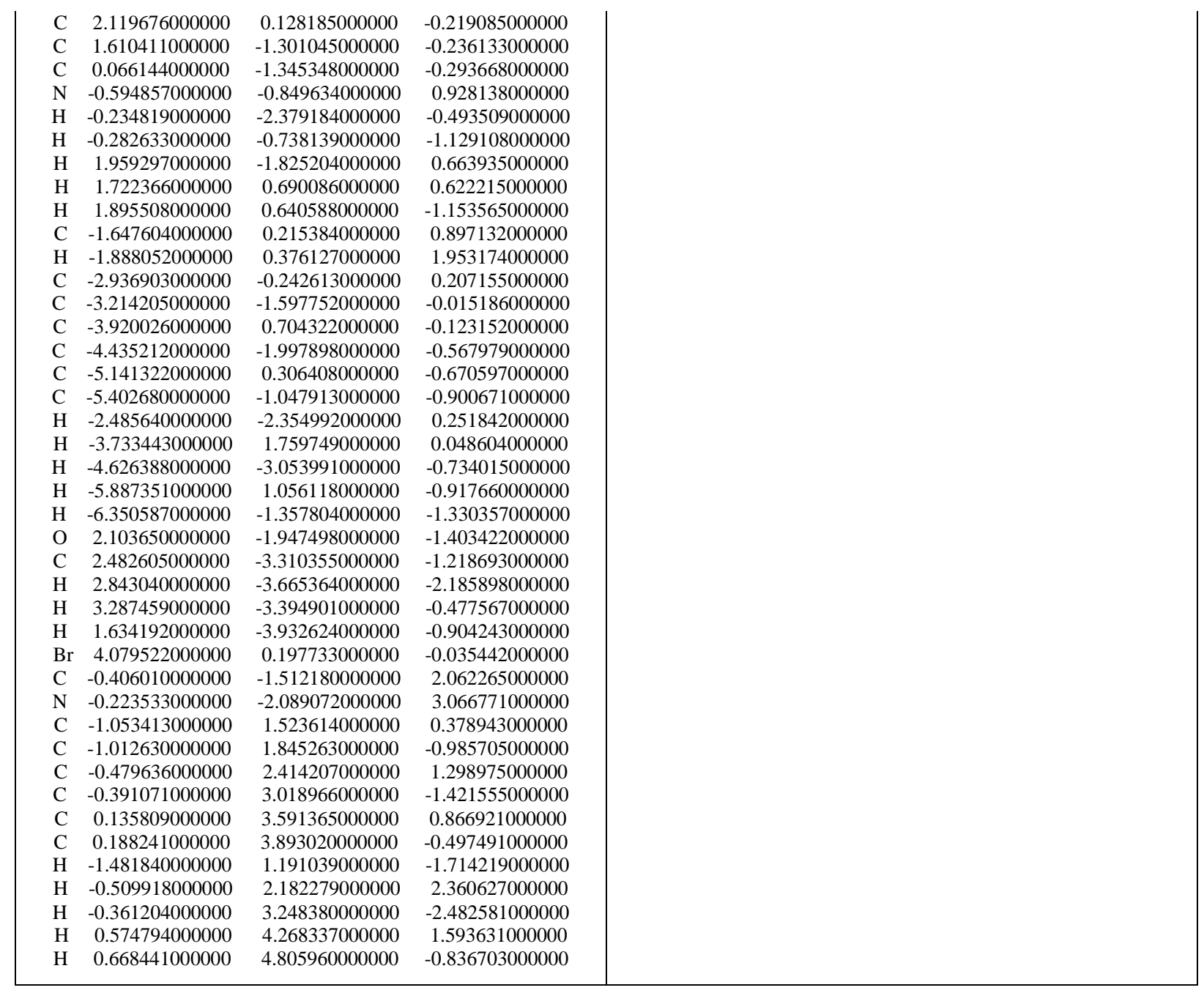

